\title{
Capturing a crucial 'disorder-to-order transition' at the heart of the coronavirus molecular pathology - triggered by highly persistent, interchangeable salt-bridges
}

\author{
Sourav Roy ${ }^{\dagger 1}$, Prithwi Ghosh ${ }^{\dagger 2}$, Abhirup Bandyopadhyay ${ }^{3}$, Sankar Basu ${ }^{4 *}$ \\ ${ }^{1}$ Dept of Microbiology and Immunology, Brody School of Medicine, East Carolina University, Greenville, NC, 27834, USA \\ ${ }^{2}$ Department of Botany, Narajole Raj College, Narajole, Paschim Medinipur, West Bengal, India \\ ${ }^{3}$ Theoretical Neurosciences Group, Institute De Neurosciences Des Systems, Aix-Marseille University, France \\ ${ }^{4}$ Department of Microbiology, Asutosh College (affiliated to University of Calcutta), 92, Shyama Prasad Mukherjee Rd, \\ Bhowanipore, Kolkata, West Bengal 700026, India \\ *Corresponding Author's Email: nemo8130@gmail.com
}

Email addresses of all authors:

Dr. Prithwi Ghosh: prithwi11@gmail.com (ORCiD: 0000-0001-5243-751X)

Dr. Sourav Roy: srvryt@gmail.com (ORCiD: 0000-0001-5354-3824)

Dr. Abhirup Bandyapadhyay: abhirupnit@gmail.com (ORCiD: 0000-0001-7395-1323)

Dr. Sankar Basu*: nemo8130@gmail.com (ORCiD: 0000-0003-1393-1982)

\begin{abstract}
The COVID-19 origin debate has greatly been influenced by Genome comparison studies of late, revealing the seemingly sudden emergence of the Furin-Like Cleavage Site at the S1/S2 junction of the SARS-CoV-2 Spike (FLCS Spike) containing its ${ }_{681} P R R A R_{685}$ motif, absent in other related respiratory viruses. Being the rate-limiting (i.e., the slowest) step, the host Furin cleavage is instrumental in the abrupt increase in transmissibility in COVID-19, compared to earlier onsets of respiratory viral diseases.

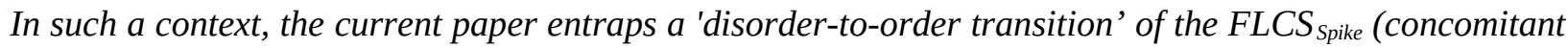
to an entropy arrest) upon binding to Furin. The interaction clearly seems to be optimized for a more efficient proteolytic cleavage in SARS-CoV-2. The study further shows the formation of dynamically interchangeable and persistent networks of salt-bridges at the Spike-Furin interface in SARS-CoV-2

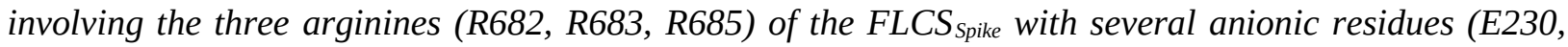
E236, D259, D264, D306) coming from Furin, strategically distributed around its catalytic triad. Multiplicity and structural degeneracy of plausible salt-bridge network archetypes seems the other key characteristic features of the Spike-Furin binding in SARS-CoV-2 allowing the system to breathe - $a$ trademark of protein disorder transitions. Interestingly, with respect to the homologous interaction in SARS-CoV (2002/2003) taken as a baseline, the Spike-Furin binding events generally in the coronavirus lineage seems to have a preference for ionic bond formation, even with lesser number of cationic residues at their potentially polybasic FLCS spike patches. The interaction energies are suggestive of a characteristic metastabilities attributed to Spike-Furin interactions generally to the coronavirus lineage - which appears to be favorable for proteolytic cleavages targeted at flexible protein loops. The current findings not only offer novel mechanistic insights into the coronavirus molecular pathology and evolution but also add substantially to the existing theories of proteolytic cleavages.
\end{abstract}

Keywords: COVID-19, SARS-CoV-2, Spike-Furin interaction, FLCS, Disorder-to-order transition, enthalpy - entropy compensation, entropy arrest, Salt-bridge dynamics, Proteolytic cleavage 


\section{Introduction}

There has been a dramatic shift [1] in latest COVID research from its early chapters (2019-20 $\rightarrow$ 2020-21) brought about by epidemiological and evolutionary (genome comparison) studies of late [2,3], reporting the presence of an arginine-rich polybasic Furin like cleavage site (FLCS) at the Spike-S1/S2 junction of SARS-CoV-2, absent otherwise in related coronavirus species. This has certainly raised doubts about the origin of SARS-CoV-2 (whether purely natural $[4,5]$ or otherwise $[1,6])$ which is presently obscure and debatable $[7,8]$. The latest understanding is that it was during a systematic 'gain of function' mutational studies [9] carried out on gradually evolving strains of the coronavirus (starting from its natural template, SARS$\mathrm{CoV}, 2003$ ) that the virus triggering the current pandemic (SARS-CoV-2) accidentally got released and that too from a highest bio-safety level virology laboratory (at the Wuhan Institute of Virology [10,11], Wuhan, China). Alarming concerns have thus been unavoidable ever since on the ethical grounds of the decades-long practices of 'gain of function' research in virology [12-14]. Apart from the presence of the FLCS in the SARS-CoV-2 Spike (FLCS ${ }_{\text {Spike: }}$ absent in other beta-CoVs) the other most striking evolutionary feature of SARS-CoV-2 has been its $\mathrm{RBD}_{\text {spike }}$ [15] which is highly optimized for binding with its human host receptor, hACE2 (among all species known to harbor homologous receptors $[16,17]$ ), thus channeling the viral influx heavily towards the human population. Both these features, collectively and on their own, present a certain degree of abruptness considering both the time-scale and the sequential nature of changes attributed to natural evolution. These features thereby are strongly argued to carry blueprints of possible human interventions, demanding for further investigations.

The $\mathrm{RBD}_{\text {spike }}$ - hACE2 interaction in SARS-CoV-2 had been well characterized by 2019-20 [1820] and so was its role in the virus host cell entry [18]. A kinetically driven "down-to-up" conformational transition of $\mathrm{RBD}_{\text {spike }}$ triggered upon a close proximity to hACE2 has been found instrumental for the virus host cell entry. This "down-to-up" transition of $\mathrm{RBD}_{\text {spike }}$ enables it to dock to the solvent exposed hACE2 molecular surface. The RBD spike $_{-}$- hACE2 interaction, reminiscent of a molecular handshake [15], serves as a molecular switch in the viral cell entry. The $\mathrm{RBD}_{\text {spike }}$ - hACE2 interface has a low electrostatic matching [15], characteristic of quasistable interactions and therefore perhaps best-fitted for (transient) molecular switches. The befitting surface docked to the solvent exposed Spike binding site of hACE2 selects solely for a

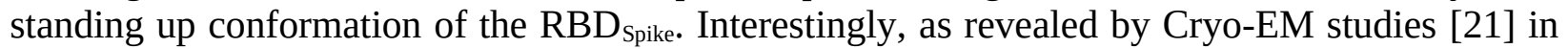
SARS-CoV, this 'up' state is the temporally prevalent state while in SARS-CoV-2, the 'up' state is only triggered in close proximity to hACE2. This, when viewed together with the abrupt emergence of host Furin cleavage and that of the polybasic FLCS $_{\text {Spike }}$ site in SARS-CoV-2 (absent in SARS-CoV) certainly appears to be contradictory to the slow and graduated changes attributed to natural evolution. The more efficient the cleavage, the more easier it would be for the virus to gain entry inside the host cell [1]. At one end, there's no FLCS spike $_{\text {in }}$ the SARS-CoV Spike which therefore is only cleaved randomly (and hence less frequently) by non-specific proteases, thereby always demands an 'up' state of the $\mathrm{RBD}_{\text {spike }}$ for the interaction to occur $[22,23]$. At the other end, the native, resting, lying down state of the $\mathrm{RBD}_{\text {spike }}$ in SARS-CoV-2 helps the virus to escape the host immune surveillance mechanisms $[24,25]$. The overall impact is interesting (and perhaps somewhat counter-intuitive) that there is an increase in binding affinity in the younger homologue (SARS-CoV-2) compared to its evolutionary ancestor (in 
SARS-CoV, 2002/2003) while in terms of binding stability (attributed to electrostatic matching at the interface) there is a 'critical' drop [26] from $\mathrm{CoV}$ to $\mathrm{CoV}-2$, imparting a bouncing nature in the later interaction and thereby making the neighboring cells more susceptible to subsequent viral entries than the earlier event (SARS-CoV).

In addition to the $\mathrm{RBD}_{\text {spike, }}$, host protease pre-activation (or priming) plays an imperative role in SARS-CoV-2 pathogenesis critically enhancing its efficient host cell entry. Notably, the priming step acts a promotional factor in SARS-CoV-2 but is non-essential (for infection and cell-cell fusions) [27] generally in the coronavirus lineage. In case of SARS-CoV-2 (and, its subsequent lately evolved variants [28-30]) the virus uses its arginine-rich FLCS $_{\text {Spike }}$ as a lucrative bait to recruit host-encoded pro-protein convertases (PC), primarily Furin [31] (the best-characterized mammalian PC [32]) rapidly at the Spike-S1/S2 junction. This then is relatively slowly [1] followed by the host Furin cleavage of the said junction (Spike-S1/S2) eventually leading to a more efficient host cell entry of SARS-CoV-2 [33] (compared to SARS-CoV and other related respiratory viruses) and its variants [1,28,30]. Apart from Furin, SARS-CoV-2 entry is also primed by a cell surface protease TMPRSS2, lysosomal cathepsins [34], and, also by proteases (NE, PR3, CatG, NSP4) released by activated neutrophils [35] swarming around the invaded pathogen to elicit an immune response. They therefore can be hijacked by virus-derived surface proteins as a mean to escape the host immune surveillance. All these eventually leads to a cumulative effect of (Furin like) host proteases on SARS-CoV-2 entry. Furin is also well known for its undue involvement in various pathologies, especially related to bacterial and other viral diseases (e.g., Anthrax, Ebola) [32]. Structurally, it is the association of two domains, (i) a catalytic domain consisting of closely packed $\alpha$-helices and intertwined crisscrossed beta strands at the $\mathrm{N}$ terminus and (ii) an all- $\beta$ (C-terminus) $\mathrm{P}$ domain. While the evolutionarily varied domain is the $\mathrm{P}$ domain, the catalytic domain is highly conserved across mammals, and, further harbors a characteristic 'Serine-Histidine-Aspartate' catalytic triad that mediates the (proteolytic) cleavage. With the help of this triad, Furin cleaves precursor proteins (or, proproteins) having a basic consensus of 'R-X-K/R-R-' like motif with stringent specificity [32]. The stringency, as well as the preference towards basic residues, has been structurally explained in Furin by the presence of contoured surface loops shaping the active site and harboring highly

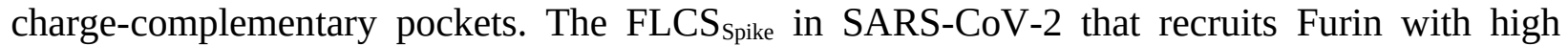
affinity has a ' ${ }_{681} P R R A R_{685}$ ' motif as the polybasic consensus, encoded by a 15 -nucleotide insert 5 -CCTCGGCGGGCACGT-3` at the Spike-S1/S2 junction. Interestingly, the two consecutive arginine residues $(R)$ in the 'PRRAR' are both encoded by a CGG codon $\left(2{ }^{\text {nd }}, 3^{\text {rd }}\right.$ triplet in the $\mathrm{ORF}^{1}$ ) - which is the most infrequent codon for viruses (out of the six codons dedicated for arginines) [1]. The presence of this unique and specific 15-nucleotide sequence in the SARS$\mathrm{CoV}-2$ genome was even satirically referred to as a "smoking gun" [1] supporting the lab-origin theory of this virus. Further, as confirmed by functional studies, the loss of FLCS Spike $_{\text {has }}$ been shown to attenuate SARS-CoV-2 pathogenesis [33]. The discovery of the unique presence of FLCS $_{\text {spike }}$ in SARS-CoV-2 has also caused a shift of scientific parlance in the subject, directing researchers from a physics- to a chemistry- observation window. The $\mathrm{RBD}_{\text {spike }}-\mathrm{hACE2}$ interaction in SARS-CoV-2 is essentially (bio-)physical, guided by the physical laws of diffusion and collision, applicable to non-covalent (van der Waals) inter-atomic interactions. While, the

1 ORF: Open Reading Frame 
next important step involves breaking of a covalent bond in the FLCS Spike $_{\text {by Furin and therefore }}$ is a chemical process [1]. Naturally, the later is much slower than the earlier, further making it (FLCS $_{\text {Spike }}$-Furin) the rate-limiting step in the viral host cell entry [1]. Insertion of the polybasic activation loop $\left({ }_{681} \mathrm{PRRAR}_{685}\right)$ in the SARS-CoV-2 Spike as opposed to other related respiratory viruses has been pinned to increased virulence, transmissibility, and pathogenesis [35-37]. This makes it critical to understand in tandem the dynamics and transition of these loops into ordered conformers upon binding to Furin (or other proteases) to pinpoint the molecular interactions in play. Such knowledge could be pivotal in expanding our understanding of how loop dynamics, interaction and stabilization forms the molecular basis of enhanced pathogenicity and virulence of SARS-CoV-2 Spike glycoproteins. Most structures of Spike glycoproteins solved till date are

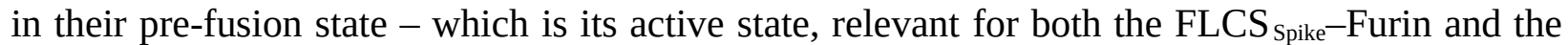
$\mathrm{RBD}_{\text {spike }}-\mathrm{hACE} 2$ interactions. The pre-fusion forms, aimed to address different specific research queries related to the coronavirus molecular pathology, are either mutated or abrogated at the FLCS $S_{\text {spike }}$ region (i.e., the Spike-S1/S2 junction) or otherwise engineered with stabilizing mutations [21,24,38], aimed at obtaining overall structural information. However, even with these stabilizing modulations, all cryo-EM coronavirus Spike structures lack experimental

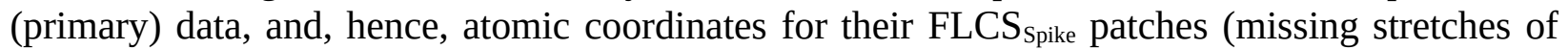
10-12 amino acids [39]) harboring the polybasic activation loop ( $\left.{ }_{681} P R R A R_{685}\right)$ in SARS-CoV-2 Spike, and, equivalent homologous pentapeptide sequence motifs in other coronavirus Spikes. The pre-fusion structure of SARS-CoV-2 Spike (PDB ${ }^{2}$ ID: 6XR8) [21] could decipher the structure of 25 ordered peptides downstream of S2 hitherto unreported including residues of $\mathrm{N}$ terminus, several peripheral loops and glycans [21]. Even this most insightful high resolution structural study, which could reveal the distinct conformational states (pre- and post-fusion forms) of the SARS-CoV-2 Spike, was unable to ascertain the conformation of the surface

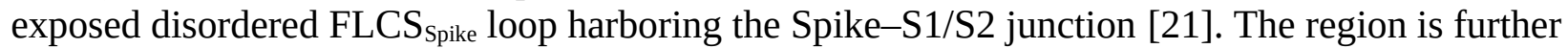
clearly and categorically declared as a "surface exposed disordered loop" [21]. The presence of such 'disordered activation loops' (also called 'natively unstructured loops' [40] or, less technically, 'flexible' loops/regions) at proteolytic sites is common to many protease families (e.g., Caspases, Furins, Rho-activated kinases etc.) and in spite of decades long controversies are still assumed to serve as key structural and kinetic determinants of protease substrates [40]. The loops, often making the substrates more susceptible to protease binding and cleavage [31], proteasomal degradation [41], phospho-regulation and consequent priming of viral pathogenesis [42] are believed to have been evolved often from sensitive and fragile globular-disorder intermediates like coiled-coil assemblies [43]. Having said that, there is little experimental structural information that is available on the cleavage loops for our current subjects, the coronavirus Spikes.

The loop-disorder is further supported by extensive (coarse-grained) multi-microsecond molecular dynamic (MD) simulations of the representative SARS-CoV-2 Spike structures (6VXX, closed state; 6VYB: partially open state, ectodomain) [36] with modeled FLCS Spike $_{\text {loops }}$ (residue ranges: 676-690) [44] that show violation of ergodic hypothesis [36], hinting towards a flexible albeit biased conformation of the activation loop for its function. In other words, during the entire course of these long MD simulations, the loops remained largely unstructured [36]

2 PDB: Protein Data Bank 
sampling several outwardly extended conformations making them attractive and accessible cleavage sites for Furin and other pro-protein convertases. So the loop disorder does not appear to be short-termed, rather dynamically sustained in the free form of the Spike. As an alternative and arguably a complementary approach, the same paper further models an ensemble of loops $a b$ initio [36] via RosettaRemodel [45] from the closed state Spike structure (6VXX). Subsequent to the modeling, followed by clustering and sorting based on energy, the lowest energy conformation was retained and further refined by kinetic closure [46]. In the conclusive remarks [36], the authors comment that the $a b$ initio modeling indicated that there might be formation of short helices near the cleavage site of the loop; however, these observations are not really supported by any data and/or geometric analysis such as the Ramachandran Plot. The very fact that the $\mathrm{FLCS}_{\text {Spike }}$ (in its ${ }_{681} P R R A R_{685}$ ) contains pockets of heavily localized positive charge cloud (a common cause of protein disorder [47]) makes it naturally and intrinsically prone to structural disorder. This makes the analysis even more interesting and non-trivial, and further implies that there needs to be a 'disorder-to-order transition' [47] of the said loop (i.e., the FLCS Spike $_{\text {region) }}$ upon interaction (or binding) with Furin. That is because the binding needs to be strong enough to sustain the disordered substrate $\left(\right.$ FLCS $\left._{\text {Spike }}\right)$ jammed into metastable intermediate conformation(s) that support the efficient cleavage of the Spike-S1/S2 junction by the Furin catalytic triad. Recent experimental studies primarily based on functional assays have referred to

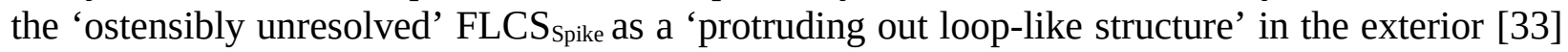

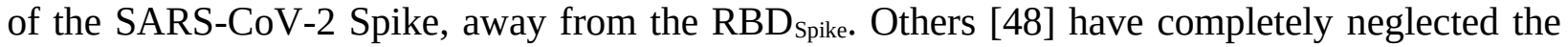
plausible conformational variation (entropy) of the missing patch(es) in their molecular docking and dynamics studies. Thus, little has genuinely been explored structurally on the Spike-Furin interaction. Given this background, here, in this paper, we present a rigorous structural dynamics study with strong theoretical rationales to penetrate deeper into the Spike-Furin interaction in SARS-CoV-2 in an atomistic detail. To the best of our knowledge, we are the first group to report on the key 'disorder-to-order transition' occurring at the SARS-CoV-2 Spike-Furin interface. Based on present knowledge and understanding, the revealed transition together with

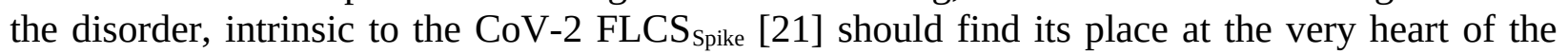
coronavirus molecular pathology. We further demonstrate that the involved 'disorder-to-order transition' in CoV-2 is triggered and sustained by highly persistent interchangeable salt-bridge dynamics - which is often characteristic to protein disorder transitions [49,50]. We also present a novel application of the legendary Ramachandran Plot [51] in probing the said 'transition' at the CoV-2 Spike-Furin interface. The conclusions should also hold true for lately evolving CoV-2 variants $[28,30]$.

\section{Materials and Methods}

\subsubsection{Details and rationales of experimental structural templates used for docking and dynamics}

Following earlier studies on the use of the SARS-CoV-2 Spike glycoprotein [15,52,53], the cryo-EM structure of its pre-fusion form (PDB ID: 6XR8, $2.9 \AA$, 25995 protein atoms [21]) was used as its representative structure (receptor). For the host protease, Furin (ligand), we first made a thorough structural survey of its domains and enzyme active sites, especially that of the catalytic domain and triad). There are only a few experimental (X-ray) structures of Furin to be found presently in the Protein Data Bank [39] (PDB ID: 1P8J, 5JXH) which are of equivalent 
resolutions with insignificant structural deviations (C $C^{\alpha}$-RMSD: $0.31 \AA$ ) upon alignment. More importantly, the region spanning Catalytic residues in both are well conserved. 1P8J, (resolution: 2.6 A), the first Furin structure ever to be solved for Furin [32] is undoubtedly the most studied and best characterized Furin structure to have, also insightful in terms of proteolytic cleavage mechanisms [48,54-57]. It is for these reasons, 1P8J was chosen as the representative host protease (Furin) structure in the current study. Again, 1P8J has 8 identical chains (pairwise RMSD $<0.2 \AA$ ) in its asymmetric unit combining to as many as 11 bio-assemblies. The majority of the bio-assemblies (bio-assemblies: 3-10) are monomeric - which is the interactive molecular species (or, functionally effective bio-assembly) involved in the Spike-Furin interaction [48]. This made us further retain the first chain (chain A) of 1P8J alone.

Further, to set an appropriate baseline for the Spike-Furin interaction in SARS-CoV-2, a second round of docking was performed by taking the same ligand (Furin: 1P8J) and docking it onto the representative homologous Spike structure (PDB ID: 7AKJ) [58] of SARS-CoV (2002/2003). This cryo EM structure was solved in the process of exploring 'the convalescent patient sera option' for the then seemed possible prevention and treatment of COVID-19, as a natural extension of SARS [59]. The neutralizing antibody Fab fragments (chains: D-H \& L in 7AKJ), used to pull down the SARS-CoV Spike, binds at the top of the Spike canopy or the Spike S1 subunit involved in receptor binding [58] - which is situated way too far from the Spike-S1/S2 interface (harboring its FLCS $_{\text {spike }}$ ) to have any realistic interference with the Spike-Furin binding. The Spike-Furin guided docking in SARS-CoV (to be discussed in section 2.3.1.3) was followed by repeating all subsequent baseline structural dynamics calculations for the SpikeFurin complex. To set this appropriate baseline is indispensable particularly for the quantitative interpretation of equilibrium thermodynamic parameters of the Spike-Furin binding, due to be discussed in later sections (section 2.6).

\subsubsection{Dataset of coronavirus Spikes}

For an evolutionary analysis of sequence based disorder predictions, a second dataset was compiled consisting of 44 experimentally solved (exclusively cryo-EM) structures (Table S1, Supplementary Materials) of the pre-fusion form CoV/CoV-2 Spike culled at a resolution of 'not worse than $3 \AA$ ' from the PDB. These coronavirus Spike structures are solved to serve different specific research objectives at various $\mathrm{pH}$ and other varying physico-chemical conditions $[17,58,60,61]$. All these structures were found not to have any experimental (primary) data for its FLCS $_{\text {Spike }}$ patch (located at their Spike-S1/S2 junctions) resulting in missing atomic coordinates (remarked under 'REMARK 465' in the corresponding PDB files) for the said patch (10-12 amino acids).

\subsection{Modeling of missing disordered loops in Spike}

To explore the interaction dynamics between SARS-CoV-2 Spike (PDB ID: 6XR8) and Furin (1P8J), first we needed to have 'all-atom' atomic models for both partners. Furin (1P8J) is much smaller (468 residues) compared to the Spike homo-trimer (3 identical chains with 1149 residues per chain) and has no missing stretches in its X-ray structure but for the terminal most residues. The Spike trimeric structure, however, being a large glycoprotein, characteristically contains missing stretches of residues (of roughly similar lengths) localized at strategic positions, 
adding up to missing coordinates for 42 amino acids in 6XR8. As discussed in the previous subsection (section 2.1.2), to have such missing stretches of residues, especially at the Spike-S1/ S2 junctions, is common to all Spikes (Table S1, Supplementary Materials) irrespective of the particular coronavirus species. These missing stretches map to flexible [36] as well as disordered [21] loop protrusions resulting from the extended internal packing involved in the trimerization of the monomeric $S$ units. The missing disordered stretches in the representative SARS-CoV-2 Spike pre-fusion structure (6XR8) were then modeled by MODELLER (v.10.1) [62] using its full-length (proteomic [63]) sequence (https://www.rcsb.org/fasta/entry/6XR8/display) obtained from the Protein Data Bank [39]. To account for the loop disorder in the modeled missing stretches (in 6XR8), an ensemble modeling approach was adapted. To that end, the 'automodel' module of MODELLER was implemented in an iterative cycle of 500 runs, producing that many conformationally non-redundant 'all-atom' trimeric Spike atomic models, only varying among themselves at the modeled missing stretches.

Missing residues for the baseline structure of SARS-CoV Spike (7AKJ) were also build in a similar fashion using MODELLER, though opting for a much reduced space of conformational sampling (50 runs of 'automodel'). The lowest energy model among these (ranked by the allatom Rosetta energy function, availed through Rosetta@home [64]) was retained as the representative 'all-atom' SARS-CoV Spike structure to be used for all subsequent baseline calculations. This sampling may be considered adequate to represent the mildly varying (reduced) conformational space of the missing FLCS $_{\text {Spike }}$ patch ( ${ }_{664}$ SLLRSTS $_{670}$, https:/www.rcsb.org/fasta/entry/7AKJ/display) in 7AKJ - which is much shorter than its homologous missing patch in 6XR8 ( ${ }_{677}$ QTNSPRRARSVA 689$)$. The FLCS ${ }_{\text {Spike }}$ in SARS-CoV is further composed mostly of either small-polar (serines, threonines) or hydrophobic (leucines) side chains with constrained rotameric variations. Noticeably, the single charged residue (R667) situated midst the 7 residue missing patch in 7AKJ is conserved (as R685) in its evolutionarily descendant sequence in 6XR8 (Figure S1, Supplementary Materials).

\subsubsection{The Spike-Furin Molecular Docking Simulations}

\subsubsection{Blind ab-initio docking in Cluspro 2.0}

Subsequent to filling up for the structural voids in the trimeric SARS-CoV-2 Spike (section 2.2), an ensemble docking approach was adapted (using 'blind docking' in ClusPro 2.0 [65]) wherein Furin (PDB ID: 1P8J, ligand) was docked $a b$ initio onto each unbound 'all atom'

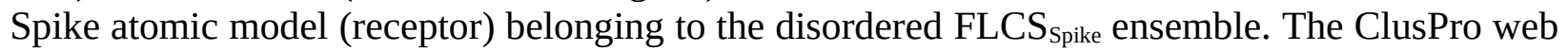
interface performs $a b$ initio blind docking in successive steps, combining course grain sampling and fine grain refinements. First, a grid-based rigid-body docking is performed between the receptor (static, fixed at the center of a cubic box) and the ligand (dynamic, placed on a movable grid) in PIPER [66] using its fast Fourier transform (FFT) correlation approach, sampling billions of conformations. The PIPER computed interaction energies (for poses sampled at each grid point) are produced in the form of correlation functions that make the scoring compatible with FFTs, rendering high numerical efficiency of sampling. The latest PIPER-derived scoring function is an upgraded variant of the original internal energy function (https://www.vajdalab.org/protein-protein-docking) which is based on the sum of terms representing shape complementarity, electrostatic, and desolvation contributions. A large number 
of unrealistic poses are then filtered out by PIPER and the 1,000 lowest-energy poses are retained. These are then undertaken to $\mathrm{RMSD}^{3}$-based structural clustering (using a relaxed $10 \AA$ cutoff for iRMSD [67]), wherein, largest clusters [65] are retained, representing the most likely poses. While the largest clusters do not necessarily contain the most near-native poses, the success rate is generally quite high across families of protein complexes [65]. Usually ten or fewer clusters ( 10-12 pose/cluster) are retained at this stage adding up to 100-120 docked poses per run. The final refinement is then performed on these selected poses by rigorous rounds of energy minimization. The blind 'ensemble docking' thus performed between Furin (ligand) and each Spike model (receptor) does not impose any additional active-site or contact residue constraints. This resultant initial pool of Spike-Furin docked poses under each docked ensemble (i.e., for each Spike model) had in them the ligand docked onto widely varying sites spread all over the trimeric Spike receptor - which were then pulled down into one unsorted set. Since the 'desired interface' would necessarily involve the FLCS ${ }_{\text {Spike, }}$, the pentapeptide motif ( ${ }_{681}$ PRRAR $_{685}$ ) was then used as 'contact residue filters' to discard obviously and/or trivially incorrect docked poses. To that end, buried surface areas (see section 2.3.2.1) and shape complementarities (see section 2.3.2.2) estimated at the desired docked interfaces were used to filter the 'plausible' docked poses in two successive rounds. These filtered 'plausible' poses were then further reranked by a carefully designed scoring function (see section 2.3.2.1) optimally combining the steric effects of the two high-level structural descriptors. Thus, in effect, the blind docking could be made to work in a guided manner. The top ranked docked pose $\left(\mathrm{RR} 1_{\mathrm{CoV}-2}\right)$ thus obtained, was further taken into long MD simulations (see section 2.4) and subsequent analyses of its structural dynamics.

\subsubsection{Cross-validation by guided docking in 'Zdock + IRaPPA re-ranking'}

The cluspro - docking was further cross-validated by Zdock with its combined feature of IRAppA re-ranking [68] implemented. To that end, the Spike chains (receptors) were extracted from the top (re-)ranked (see section 2.3.1.1) cluspro - docked pose, RR1 $1_{\mathrm{CoV}-2}$, and, Furin (1P8J, ligand) was docked onto it with the three arginines (R682, R683, R685, from the first of the three symmetry-related identical Spike chains) pertaining to the ${ }_{681} \mathrm{PRRAR}_{685}$ motif (in $\mathrm{FLCS}_{\text {spike }}$ ) specified as contact residues on the receptor molecule. No contact residues were specified from the ligand molecule. The returned top ranked model $\left(\mathrm{ZR} 1_{\mathrm{Cov}-2}\right)$ was retained and further simulated (see section 2.4) for all subsequent structural dynamics analyses.

\subsubsection{Setting up appropriate baselines: The SARS-CoV Spike-Furin guided docking in 'Zdock + IRaPPA re-ranking'}

In continuation to the earlier discussion (in section 2.1.1) on setting up appropriate baselines to interpret the Spike-Furin interaction in SARS-CoV-2, Furin (1P8J, ligand) was docked onto the SARS-CoV Spike (7AKJ, receptor) in Zdock (+IRaPPA re-ranking) in yet another independent docking exercise. To that end, a direct guided mode of docking (similar to $\mathrm{ZR} 1_{\mathrm{CoV}-2}$ ) was adapted, wherein the whole $\mathrm{FLCS}_{\text {Spike }}$ patch (residues originally missing in the

3 RMSD: Root mean square deviations 
experimental structure of 7AKJ) was specified as plausible contact residues on the Spike

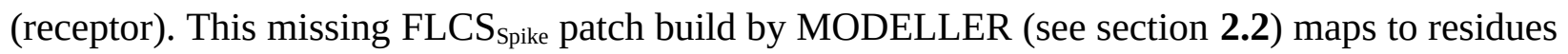
664-670 (664 SLLRSTS $_{670}$ ) and those pertaining to the first of the three symmetry-related identical Spike chains were specified as the Spike contact residues for Furin. Again, (in the same spirit to that of $\left.\mathrm{ZR} 1_{\mathrm{Cov}-2}\right)$ no contact residues were specified from Furin. The returned top ranked model $\left(\mathrm{ZR} 1_{\mathrm{Cov}}\right)$ was retained, simulated (see section 2.4) and used as a baseline for all subsequent structural dynamics calculations, probing for the absence of the 'PRRAR' motif in FLCS ${ }_{\text {Spike. }}$

\subsubsection{Docking scoring, ranking and re-ranking}

\subsubsection{Buried Surface Area calculations}

For initial filtering of (Spike-Furin) docked poses (ClusPro 2.0) the atomic accessible surface areas (ASA) were calculated by NACCESS [69] traditionally following the Lee and Richards algorithm [70] for all heavy atoms pertaining to each partner protein molecule (Furin and Spike) in their bound and unbound forms. For the unbound form, the two partner molecules in the bound docked-pose were artificially physically separated and each of them was considered independently, in isolation. The atomic accessibilities were then summed up for their source residues. For each $\left(i^{\text {th }}\right)$ residue belonging to a docked pose, two ASA(i) values were obtained, one for its bound form (ASA bound $(i))$ and the other for its unbound form (ASA unbound $(i))$. A residue falling in the interface would thus have a net non-zero change ( $\triangle \mathrm{ASA}(\mathrm{i}) \neq 0)$ in its two ASA(i) values. In other words, these would be the residues at the interface which gets buried upon complexation and are characterized by a net non-zero buried surface area (BSA(i) $>0$ ).

$$
B S A(i)=\Delta A S A(i)=\left|\left(A S A_{\text {bound }}(i)-A S A_{\text {unbound }}(i)\right)\right|
$$

For the case of Spike-Furin docking (SARS-CoV-2), BSA was used as an initial filter to select those interfaces alone that harbors the FLCS $_{\text {Spike }}$ loop. In the process, the obviously incorrect poses with Furin docked elsewhere in the Spike were discarded. This was achieved by monitoring $\mathrm{BSA}_{\mathrm{PRRAR}}$, the summed up BSA for the residues belonging to the pentapeptide ${ }_{681} \mathrm{PRRAR}_{685}$ motif. The poses that have $\mathrm{BSA}_{\mathrm{PRRAR}}>0$ were then filtered and retained from the initial pool of $a b$-initio docked poses.

BSA $_{\text {PRRAR }}$ for this BSA - filtered set of docked poses was further normalized by the total $\triangle \mathrm{ASA}$ (summed over all residues pertaining to the docked pose) to render the normalized buried surface area for the docked pentapeptide surface patch (nBSA $\left.A_{\text {PRRAR }}\right)$. The normalization can be expressed by the following equation.

$$
n B S A_{\text {PRRAR }}=\frac{B S A_{\text {PRRAR }}}{\sum_{\text {resi }} \Delta A S A_{\text {Spike }}+\sum_{\text {resi }} \Delta A S A_{\text {Furin }}}
$$

where, although, 'resi' stands for all residues pertaining to the protein chain ('subscripted' to the corresponding $\triangle \mathrm{ASA}$ sum-over term), it is the interfacial residues $(\triangle \mathrm{ASA}(\mathrm{i}) \neq 0)$ that alone actually contribute to the denominator. The use of nBSA defined at protein-protein interfaces 
[71] can be considered analogous to that of the surface overlap parameter [72,73] which has been used extensively in tandem with shape complementarity to study packing within protein interiors.

Wherever applicable, burial (bur) of solvent accessibility for a protein residue (X) was computed (following standard methods [72-75]) by taking the ratio of its ASA when embedded in the protein to that when in a Gly-X-Gly tripeptide fragment with its fully extended conformation.

$$
\operatorname{bur}(X)=\frac{A S A(X)_{\text {protein }}}{A S A(X)_{G l y-X-G l y}}
$$

Standard binning techniques for residues based on burial [72-75] were then adapted, with an ever-so-slight modification (opting for 3 instead of 4 burial bins) based on the current requirement. A protein residue based on its burial (defined in the range of $[0,1]$ ) could thus be classified into one of the three 'burial' classes: (a) buried $(0.0 \leq$ bur $\leq 0.05)$, (b) partially exposed $(0.05<$ bur $\leq 0.30)$ or $(\mathrm{c})$ exposed $($ bur $>0.30)$.

The analysis of residue-wise burial was particularly intended to survey the Furin structure, in

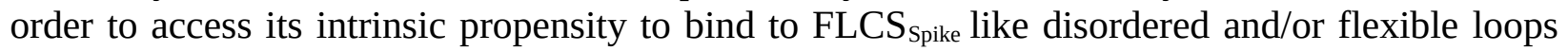
that harbors patches of highly localized and dense positive charge clouds (e.g., the 'PRRAR' pentapeptide motif).

\subsubsection{Shape Complementarity}

For a chosen docked pose which has passed the initial BSA filter (section 2.3.2.1), the shape complementarity [76,77] at its interface was computed by the shape correlation (Sc) statistic originally proposed, formulated and programmed (as the sc program, part of the CCP4 package [78]) by Lawrence and Colman [76]. Sc is a correlation function defined in the range of -1 (perfect anti-complementarity) to 1 (perfect complementarity). It elegantly combines both the alignment as well as the proximity of interacting surfaces and is essentially local in nature (resulting from Van der Wall's packing). Higher the Sc, better the packing at the interface. Wellpacked protein-protein interfaces (irrespective of their biological origin and size) usually hit a thin optimal range of Sc values ( 0.55-0.75) [15,76].

For the case of Spike-Furin docking (SARS-CoV-2), Sc was computed for the BSA - filtered (see section 2.3.2.1) interfaces alone which refers to those docked poses that harbors the FLCS $_{\text {spike }}$ loop in its interface. Furthermore, for Sc, we narrowed down our observation window

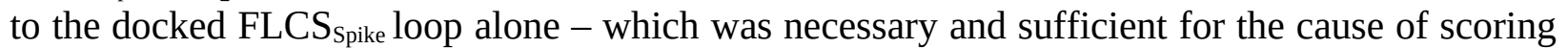
and re-ranking the initially selected (plausible) docked poses. To that end, Sc of the corresponding surface patch $\left(\mathrm{Sc}^{\mathrm{FLCS}}\right)$ was computed against its docked surface patches (combined) coming from Furin. Sc is local in nature and can be directly computed for and segregated among pairs of interacting surface patches in multi-body interactions [72,73]. To render an accurate $\mathrm{Sc}^{\mathrm{FLCS}}$ for each chosen docked pose, the sc (CCP4) [78] - input file was made to retain coordinates of the corresponding docked patch (resi. 677-688) alone for the interacting Spike chain, appended with coordinates for all atoms pertaining to the docked Furin molecule. 


\subsubsection{The $S_{\text {dock }}$ score}

The $\mathrm{S}_{\text {dock }}$ score, designed for the purpose of re-ranking of BSA - filtered (plausible) $a b$ initio docked poses was computed by the following equation.

$$
S_{\text {dock }}=\sqrt{w_{1} \cdot\left(n B S A_{P R R A R}\right)^{2}+\left(S c^{F L C S}\right)^{2}} ; w_{1}=\frac{\operatorname{median}\left(\left\{n B S A_{P R R A R}\right\}\right)}{\operatorname{median}\left(\left\{S c^{F L C S}\right\}\right)}
$$

- where, $\mathrm{w}_{1}$ (=5.78) appropriately weighted the two components (nBSA, Sc) of the score. In effect, the $S_{\text {dock }}$ score elegantly combined the surface fit and overlap at the Spike-Furin interface. The BSA - filtered (plausible) poses were then scored and re-ranked by $S_{\text {dock. }}$ The design of $S_{\text {dock }}$ can be considered analogous to deriving the magnitude of the resultant of two mutually orthogonal vectors in 2D Euclidean space.

\subsection{Molecular Dynamic Simulations}

The top ranked docked SARS-CoV-2 Spike - human Furin complex $\left(\mathrm{RR} 1_{\mathrm{CoV}-2}\right)$ consisting of 60224 atoms (inclusive of Hydrogens) were used as the initial structural template for an explicit water all atom Molecular Dynamics (MD) simulation run. All MD simulations were performed using Gromacs v2021.3 [79] with OPLS-AA force field [80]. Force-field parameters for the surface-bound glycans of SARS-CoV-2 Spike (6XR8) were used directly from a recent earlier study on the same protein from this laboratory [26], while those for SARS-CoV Spike (7AKJ) were built in an identical manner using the glycoprotein builder at GLYCAM-Web (www.glycam.org) [81]. Cubic box of edge dimension $225.1 \AA$, solvated by a total $\left(\mathrm{N}_{\text {sol }}\right)$ of 348608 TIP3P water molecules was used to solvate the protein complex with an application of periodic boundary conditions of $10 \AA$ from the edge of the box. The system was then chargeneutralized $\left(\mathrm{N}_{\text {ion }}\right)$ with $21 \mathrm{Na}^{+}$ions by replacing the TIP3P waters. The size of the hydrated system thus amounted to 899539 (protein+non-protein) atoms. Bond lengths were constrained by LINCS algorithm [82] and all long-range electrostatic interactions were determined using the smooth particle mesh Ewald (PME) method [83]. Energy minimization was performed with steepest descent algorithm until convergence ( 1000 steps) with a maximum number of steps set to 50000. All simulations were performed at 300K. Temperature equilibration was conducted by the isochoric-isothermal NVT ensemble (constant number of particles, volume, and temperature) with a Berendsen thermostat [84] for 100 ps. The system was then subjected to pressure equilibration in the NPT ensemble (constant number of particles, pressure, and temperature) for 100 ps using the Parrinello-Rahman protocol [85], maintaining a pressure of 1 bar. Coordinates were written subsequent to necessary corrections for Periodic Boundary Conditions (PBC) using the GROMACS command 'gmx trjconv' using its -pbc option. Backbone RMSDs were computed using the GROMACS command 'gmx rms' and monitored throughout the trajectory. For $\mathrm{RR} 1_{\mathrm{CoV}-2}$, the production run was set to 300 ns which may be considered sufficiently long as the system showed convergence. For cross-validation purposes, a second MD simulation was set 
up with $\mathrm{ZR} 1_{\mathrm{CoV}-2}$ as the template (see section 2.3.1.2) and run for $100 \mathrm{~ns}$ with identical cubic box dimensions, $\mathrm{N}_{\text {sol }}$ and $\mathrm{N}_{\text {ion }}$. To set up appropriate baselines, yet another third independent simulation was set up and run for $100 \mathrm{~ns}$ using $\mathrm{ZR} 1_{\mathrm{Cov}}$ as the template (see section. 2.3.1.3) with cubic box of edge dimension $190.6 \AA$, solvated by 214418 water molecules and chargeneutralized by $45 \mathrm{Na}^{+}$ions. All three simulation trajectories attained equilibrium, ensuring seamless downstream calculations. For subsequent analyses of structural dynamics, structural snapshots were extracted at a regular interval of $10 \mathrm{ps}$ from all three trajectories leading to 30000 snapshots for $\mathrm{RR} 1_{\mathrm{CoV}-2}$ (the subject), 10000 snapshots for $\mathrm{ZR} 1_{\mathrm{CoV}-2}$ (the subject for crossvalidation) as well as $\mathrm{ZR} 1_{\mathrm{Cov}}$ (the baseline). All simulations were performed on a local workstation with Gromacs v2021.3 [79] with CUDA acceleration v11.2 powered by an NVIDIA RTX 3080 GPU with 8704 CUDA compute cores resulting in an average output simulation trajectory of $\sim 8.4 \mathrm{~ns} /$ day.

\subsubsection{Identifying Salt-bridges at the Spike-Furin interface}

For each selected Spike-Furin interface which could either belong to the static ensemble of top ranked docked poses (see section 2.3.2) or time-frames/snapshots pertaining to MD simulation trajectories (see section 2.4) produced from top ranked selected docked poses, first, its interfacial inter-residue contact map was extracted. An interfacial inter-residue contact at the said interface was defined and detected when two heavy atoms, one coming from a Spike- and one coming from a Furin- residue was found within $4.0 \AA$ of each-other.

Further, from this interfacial contact map, ionic bonds / salt-bridges were identified following standard definitions and computational techniques [49,50,86,87]. To that end, those inter-residue contacts were assembled and characterized as salt-bridges / ionic bonds where two oppositely charged side chain heavy atoms, a nitrogen $\left(\mathrm{N}^{+}\right)$and an oxygen $\left(\mathrm{O}^{-}\right)$, coming from two different amino acid residues from the two molecular partners (Spike and Furin) were found within $4.0 \AA$ of each-other. In a salt-bridge thus formed, the positively charged nitrogen refers to side chain amino groups $\left(-\mathrm{NH}_{3}{ }^{+} /=\mathrm{NH}_{2}{ }^{+}\right)$of lysines / arginines / doubly protonated histidines (His + ) while the negatively charged oxygen refers to side chain carboxylic groups (- $\left.\mathrm{COO}^{-}\right)$of glutamates / aspartates.

\subsubsection{Analyzing salt-bridge dynamics}

\subsubsection{Salt-bridge Persistence and Occurrence}

Following standard analytical methods [49,50], first, all unique salt-bridges occurring at least once in the MD simulation trajectories were identified and accumulated. The dynamic persistence (pers) of each unique (non-redundant) salt-bridge was then calculated as the ratio of the number of structural snapshots to which the salt-bridge was found to form with respect to the total number of snapshots sampled (at regular intervals) in the trajectory. As has been already mentioned (section 2.4) an interval of 10 ps was chosen, leading to 30000 snapshots for the 300 ns trajectory $\left(\mathrm{RR} 1_{\mathrm{CoV}-2}\right)$ and 10000 snapshots for the 100 ns trajectories $\left(\mathrm{ZR} 1_{\mathrm{CoV}-2}\right.$ : crossvalidation, $\mathrm{ZR} 1_{\mathrm{Cov}}$ : baseline). Likewise, for the static ensemble compiled of the top ranked 100 docked poses $\left(\mathrm{RR} 1_{\mathrm{CoV}-2}\right.$ to $\left.\mathrm{RR} 100_{\mathrm{CoV}-2}\right)$, a static equivalent of persistence, namely, occurrence 
(occ) was procured for each salt-bridge occurring at least once in the ensemble using an equivalent ratio to that of the dynamic persistence (pers). Occurrence (occ) for each salt-bridge was defined as the ratio of docked poses to which the salt-bridge had occurred with respect to the total number of selected docked poses $(=100)$ in the ensemble. Even a single occurrence of a salt-bridge in an ensemble was considered accountable in this analysis. Normalized frequency distributions of salt-bridge persistence (and occurrence) were plotted for the corresponding ensembles for further analyses.

\subsubsection{Average Contact Intensities of salt-bridges}

To take care of the variable degrees of intensities (effectively, ionic strengths) of atomic contacts for salt-bridges, contact intensities (CI) were defined and computed for each salt-bridge as the actual number of inter-atomic contacts involved in a salt-bridge. In other words, $\mathrm{CI}$ is the number of ion-pairs to be found within $4 \AA$ between the two interacting side chains in a saltbridge. Considering all unique combinations of possible salt-bridges (Arg $\leftrightarrow$ Glu, Lys $\leftrightarrow$ Asp etc.), CI can vary from 1 to 4 . Time series averages, defined as the average contact intensity (ACI) of these salt-bridges were then computed for each non-redundant salt-bridge from the MD simulation trajectories pertaining to each subject under test (RR1 $\left.1_{\mathrm{Cov}-2}, \mathrm{ZR} 1_{\mathrm{CoV}-2}, \mathrm{ZR} 1_{\mathrm{Cov}}\right)$. Together persistence (pers) and average contact intensity (ACI) can be considered as 'ensemble descriptors of salt-bridges'. To account for their cumulative contribution in terms of salt-bridge strength and sustenance, a weighted persistence term (wpers(i)) was further defined for each $\mathrm{i}^{\text {th }}$ non-redundant salt-bridge in an ensemble, as the direct product of pers(i) and ACI(i).

$$
\text { wpers }(i)=\operatorname{pers}(i) \cdot A C I(i)
$$

By definition, wpers would have a theoretical range of $[0,4]$.

Furthermore, in order to draw a direct comparison between cumulative contact intensities (CCI) of the ionic bond networks formed across the different Spike-Furin interfaces in SARS-CoV $\left(\mathrm{ZR} 1_{\mathrm{CoV}}\right)$ and SARS-CoV-2 (RR1 $\left.1_{\mathrm{CoV}-2}, \mathrm{ZR} 1_{\mathrm{CoV}-2}\right)$ the following sum-over measure was defined, designed and implemented.

$$
C C I=\sum_{i=1}^{N} \operatorname{pers}(i) \cdot A C I(i)
$$

- where, pers(i) and ACI(i) were defined as before (in Eqn. 5) and N is the total number of nonredundant salt-bridges found at least once in a dynamic ensemble. In the current context, CCI can be considered as a measure of structural degeneracy [88] that is made to function as a global network descriptor raising a limiting threshold at the coronavirus Spike-Furin interfaces, allowing, absorbing and accommodating different alternative ionic bond network architectures as long as they are befitting to the task of catalyzing the Spike-S1/S2 cleavage. 


\subsection{Calculation of structure-based equilibrium thermodynamic parameters $(\Delta H, \Delta S, \Delta G)$ for the Spike-Furin binding}

As a mean to probe a highly likely event of 'enthalpy - entropy compensation' associated implicitly with the the Spike-Furin interaction, structure-based equilibrium thermodynamic parameters $(\Delta \mathrm{H}, \Delta \mathrm{S}, \Delta \mathrm{G})$ were calculated for the selected representative structure $\left(\mathrm{RR} 1_{\mathrm{CoV}-2}\right)$ along its entire MD simulation trajectory (300 ns) using the standalone (C++ with boost library) version (v.4) of FoldX (http://foldxsuite.crg.eu/) [89,90]. FoldX has its energy terms carefully parameterized by actual experimental data from protein engineering studies [89] - which together with its high computational speed are definite edges over the traditional $\mathrm{MM}(\mathrm{PB} / \mathrm{GB}) \mathrm{SA}$ approaches [91]. It is for these reasons, FoldX is slowly but surely taking over traditional approaches in structure-based thermodynamic calculations, particularly in the domain of protein engineering and stability analysis [92,93]. FoldX is built on a 'fragment-based strategy' that exploits the power of fragment libraries [94] in the same direction to that of the most compelling 'fragment assembly simulated annealing' approach in protein structure prediction attributed to David Baker and Rosetta [64]. Along with net free-energy changes ( $\Delta \mathrm{G}_{\text {binding/folding }}$ ) the advanced empirical force field of FoldX also returns a plethora of different favorable or disfavored transition enthalpic as well as entropic energy terms for proteins (folding) and PPI complexes (binding) directly from their high-resolution 3D coordinates (using full atomic description). To address the plausible 'enthalpy - entropy compensation' in the current context, as enthalpic terms we included the favorable van der Waals $\left(\Delta \mathrm{H}_{\mathrm{vdwF}}\right)$ and electrostatic $\left(\Delta \mathrm{H}_{\text {electro }}, \Delta \mathrm{H}_{\text {elec-kn }}\right)$ contributions to free energy, as well as the disfavored van der Waals clashes $\left(\Delta \mathrm{H}_{\mathrm{vdw} \text {-clash}}, \Delta \mathrm{H}_{\mathrm{vdw} \text {-clash-backbone }}\right)$; while, to account for the entropic costs, we included the entropic energies for backbone $\left(\mathrm{T} \Delta \mathrm{S}_{\mathrm{mc}}\right)$ and side chain $\left(\mathrm{T} \Delta \mathrm{S}_{\mathrm{sc}}\right)$ conformational changes. The choice of the terms was guided by well-just reviews and discerning followup studies on FoldX $[92,95]$. The enthalpic terms were further summed up according to the nature of forces giving rise to each.

$$
\begin{aligned}
& \Delta H_{v d w}=\Delta H_{v d w F}+\Delta H_{v d w-\text { clash }}+\Delta H_{v d w-\text { clash-backbone }} \\
& \Delta H_{\text {elec }}=\Delta H_{\text {electro }}+\Delta H_{\text {electro-kn }}
\end{aligned}
$$

To that end, structural snapshots were sampled at 10 ps interval from the 300 ns MD simulation trajectory (RR1 $\left.1_{\mathrm{Cov}-2}\right)$ resulting in 30000 time-stamps (or, structural snapshots). Then, for each snapshot, FoldX was run using the command AnalyseComplex with the complexWithDNA parameter set to 'false' and the relevant enthalpic $\left(\Delta \mathrm{H}_{\mathrm{vdw}}, \Delta \mathrm{H}_{\text {elec }}\right)$, entropic $\left(\mathrm{T} \Delta \mathrm{S}_{\mathrm{mc}}, \mathrm{T} \Delta \mathrm{S}_{\mathrm{sc}}\right)$ and free energy terms ( $\left.\Delta \mathrm{G}_{\text {binding }}\right)$, as detailed above, were computed for each run, indexed appropriately and stored. Time averages (denoted by angular braces ' $<>$ ' throughout the paper) were computed for each individual term along with its standard deviations (SD). For a second analyses, focusing purely on the 'entropy arrest' [96-98] presumably implicit to the Spike-Furin binding, conformational entropies for backbone (subscripted as 'mc') and side chains (subscripted as 'sc') were recorded (for each time-stamp) independently for the FoldX-separated (unbound) receptor ( $\Delta S_{m c}^{\text {receptor }}, \Delta S_{s c}^{\text {receptor }}$ ) and ligand $\left(\Delta S_{m c}^{\text {ligand }}, \Delta S_{s c}^{\text {ligand }}\right.$ ), as well as in their bound forms ( $\Delta S_{m c}^{\text {complex }}, \Delta S_{s c}^{\text {complex }}$ ). 
Lastly, from the individual time-series averages of the $\Delta \mathrm{G}_{\text {binding }}$ values obtained for the SpikeFurin binding in SARS-CoV and SARS-CoV-2, $\Delta \Delta \mathrm{G}_{\text {binding }}$ was defined as follows taking care of the cumulative effect of mutational changes at their FLCS ${ }_{\text {Spike. }}$.

$$
\Delta \Delta G_{b i n d i n g}=\Delta G_{b i n d i n g}^{S A R S-C o V 2}-\Delta G_{b i n d i n g}^{S A R S-C o V}
$$

\subsection{Ramachandran Plot (RP) - derived parameters to probe state-transitions (e.g., disorder-to- order)}

The Ramachandran Plot (RP) [51] can effectively be used to probe transitions between disordered and relatively ordered protein structural elements. To achieve this, first, dynamic conformational ensembles need to be assembled, representative of two protein states, say, an unbound (highly disordered) and a bound (relatively ordered) state. Since, RP is essentially based on local steric clashes, the analysis can further be locally restricted to a 'contiguous region of interest' (or, a concerned structural patch) wherein residues are supposed to undergo a twostate transition (say, disorder-to-order). In the current study, this 'contiguous region of interest'

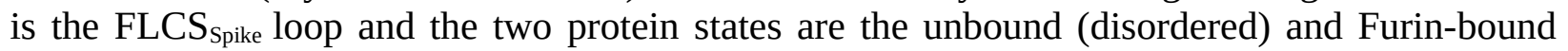
(presumably more ordered) states of the SARS-CoV-2 Spike. The Ramachandran angles $(\Phi, \psi)$ can then be computed for residues comprising the concerned structural patch and plotted in an RP for each atomic model / frame in an ensemble state. The individual RPs can then be overlaid for 'disordered' or 'relatively ordered' states. In order to identify whether a connected structural patch (especially, relevant for protein loops) supports a finite set of (restrained) structural conformations, the 2-dimensional euclidean distance in the $\Phi-\psi$ vector space of RP were computed for each internal residue comprising the concerned structural patch. The distance between Ramachandran angles of $\mathrm{i}^{\text {th }}$ and $\mathrm{j}^{\text {th }}$ residues in $\Phi-\psi$ space can be interpreated in terms of the extent of local conformational mismatches between $\mathrm{i}^{\text {th }}$ and $\mathrm{j}^{\text {th }}$ residues and may be formally represented as follows:

$$
\delta(i, j)=\sqrt{\left(\left(\phi_{i}-\phi_{j}\right)^{2}+\left(\psi_{i}-\psi_{j}\right)^{2}\right)}
$$

The maximum value of $\delta(i, j)$ represents the maximum spread of $(\Phi, \psi)$ within the concerned structural patch. It naturally follows that the concerned patch is structurally relatively more ordered when this spread is comparatively less and vice-versa. For some statistical reference, say $<\delta>_{9 \mathrm{~d}}$ be the $9^{\text {th }}$ decile of $\delta(\mathrm{i}, \mathrm{j})$. This indicates that $90 \%$ of the residues are separated by some distance less than $\langle\delta\rangle_{9 \mathrm{~d}}$ in the $\{\Phi, \psi\}$ space. Consequently, higher structural order is reflected through lesser values of $\langle\delta\rangle_{9 \mathrm{~d}}$ which increases with the increasing disorder in the structure. We took three statistics from the distribution of these distances $\delta(\mathrm{i}, \mathrm{j})$ obtained for each state: (i) the median (50 percentile, $<\delta>_{\text {median }}$ ), (ii) the $3^{\text {rd }}$ quartile (75 percentile, $<\delta>_{3 q}$ ) and (iii) the $9^{\text {th }}$ decile (90 percentile, $<\delta>_{9 \mathrm{~d}}$ ) which are adequate to collectively render a comparison across states. Use of such a combined statistics instead of the maximum value of $\delta(i, j)$ also implicitly avoid possible outlier effects. 
It is also necessarily important to estimate the local coherence of structural conformation within the concerned structural patch in general. Such a measure (metric) could be informative in terms of the local tendencies within (say) a protein loop to attain certain restrained local structural conformations. To estimate local structural coherence within the concerned structural patch, the average euclidean distance (along with the standard deviation) of $(\Phi, \psi)$ points between consecutive residues comprising the concerned structural patch was designed and computed in the following way:

$$
\left|\delta_{c}\right|=\frac{1}{N-1} \sum_{i=1}^{N-1} \delta(i, i+1) \text { and } \sigma\left(\delta_{c}\right)=\sqrt{\frac{\sum_{i=1}^{N-1}\left(\delta(i, i+1)-\left|\delta_{c}\right|\right)^{2}}{N-1}}
$$

where $|\delta c|$ and $\sigma\left(\delta_{c}\right)$ are defined for each atomic model / frame falling within an ensemble (state) and the 'contiguous region of interest' (or concerned structural patch) is N-residues long. Relative lower values of $\left|\delta_{c}\right|$ represents higher local structural coherence and $\sigma\left(\delta_{c}\right)$ presents a measure of dispersion in local structural coherence within a protein loop. Hence, these ordered parameters may be computed to collectively render a comparison of local structural coherence across states.

\subsection{Quantifying a change between two $N$-binned frequency distributions and assessing its statistical significance in terms of $\chi^{2}$}

A $\chi^{2}$ test (wherever applicable) was conducted to discriminate between two frequency distributions (say, that of an unbound and a bound state of a protein region spanning different contoured regions the RP) with the $X^{2}$-statistic being computed (for an N-bin model; $\mathrm{df}^{4}=\mathrm{N}-1$ ) by the following equation.

$$
\chi 2=\sum_{i=1}^{N} \frac{(E(i)-O(i))^{2}}{E(i)}
$$

- where $E(i)$ represents the frequency 'under the null hypothesis' expected for the $\mathrm{i}^{\text {th }}$ bin, while, $\mathrm{O}(\mathrm{i})$ denotes the actually observed frequency for that same $\left(\mathrm{i}^{\text {th }}\right)$ bin.

\section{Results and Discussion}

\subsection{Structural insight into the Furin cleavage mechanisms}

From a structural, biochemical as well as from a biophysical perspective, it is crucial to unravel the reaction mechanisms and the involved enzyme kinetics of the Furin cleavage demanding quantum chemical and/or $\mathrm{QM} / \mathrm{MM}^{5}$ studies. To that end, a preceding step would be to explore the nature of binding involved in the Spike-Furin interactions via the disordered

4 df: degree of freedom

5 QM/MM: Quantum Mechanics / Molecular Mechanics 
FLCS $_{\text {Spike }}$ - loops in SARS-CoV and SARS-CoV-2, compare them and contrast. To address this, here we adapted a combined approach of ensemble molecular docking and dynamic simulations followed by conformational analyses. As briefed in the Introduction, the coronavirus Spike

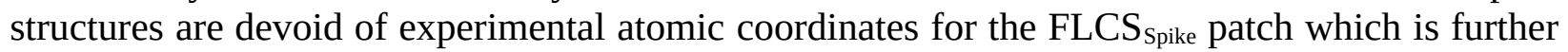
revealed to be a "surface exposed disordered loop" [21] for the pre-fusion structure of SARSCoV-2 Spike. There are as many as 44 experimentally solved (exclusively cryo-EM) structures (Table S1, Supplementary Materials) currently to be found in the PDB (see section. 2.1.2, Materials and Methods) for the pre-fusion form CoV/CoV-2 Spike, culled at a resolution of not worse than $3 \AA$. These coronavirus Spike structures are solved to serve different specific research objectives at various $\mathrm{pH}$ [99] and other varying physico-chemical conditions [17,58,60,61], therefore, often requiring stabilizing (engineered) mutations at the FLCS ${ }_{\text {Spike }}$ patches [24,38]. It is almost intriguing that even with stabilizing modulations, there's not a single cryo-EM structure that has any experimental (primary) data for its FLCS $_{\text {spike }}$ patch. As a result, atomic coordinates of the ${ }_{681} P R R A R R_{685}$ motif in the SARS-CoV-2 Spike (and, equivalent homologous sequence motifs from other coronavirus Spike) along with short flanking regions at both ends (adding up to a stretch of 10-12 amino acids) are missing experimentally [39] and hence require computational modeling (Figure S2, Supplementary Materials). To have such disordered loops appears quite characteristic of the SARS-CoV-2 Spike trimer which contains a total of four missing stretches of roughly similar lengths at strategic positions, adding up to 42 amino acids in PDB ID: 6XR8. This is perhaps reasonable given the extended internal packing involved in the trimerization of the monomeric $S$ units. The highly localized positive charge cloud concentrated over the arginine-rich ${ }_{681}$ PRRAR $_{685}$ region of the loop further boosts the said probability as this would instigate electrostatic self-repulsion of the loop adding to its conformational instability. The presence of Proline (P681), the well-known helix breaker, within the SARS-CoV-2

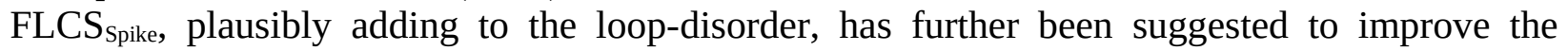
protease active site accessibility for Furin as well as for other proteases [100].

\section{2. 'More the arginines, more the disorder' in the FLCS ${ }_{\text {spike }}$ activation loops}

In order to have a general idea as to how the presence of polybasic sequences (arginines) influence the evolutionarily manifested disorder in the FLCS $_{\text {spike, we started the proceedings with }}$ an evolutionary analysis of the loop-disorder on compiled coronavirus Spike sequences. There are several $\mathrm{AI}^{6}$-trained sequence based disorder predictors [101-103] that return residue-wise disorder probability scores, which are trained primarily on evolutionary sequence data (e.g., mutational co-variance matrices). These sequence-based disorder predictors have their known limits in accuracy [104,105], for not explicitly accounting for the actual three-dimensional structural dynamics of the protein(s) / peptide(s), but, can serve as a good first test of the comparative FLCS $_{\text {Spike }}$ loop-disorder among its close evolutionary homologs. A representative set of Spike structures (CoV/CoV-2) were culled (resolution $\leq 3 \AA$ ), accumulated (see subsection 2.1, Materials and Methods), and their UNIPROT sequences (in FASTA format) derived from proteomics data [63], were extracted from corresponding entries in the Protein Data Bank [39]. The full-length Spike sequences were aligned using MUSCLE [106] and those containing gap(s)

6 AI: Artificial Intelligence 
at their aligned position(s) homologous to the ${ }_{681}$ PRRAR $_{685}$ pentapeptide motif (FLCS ${ }_{\text {spike, }}$ SARS$\mathrm{CoV}-2)$ were removed. The final set consisted of all unique and non-redundant pentapeptide sequence motifs (PSGAG, PGSAS, PASVG, PSRAG, PSRAS, PRRAA, PRARR) to be found

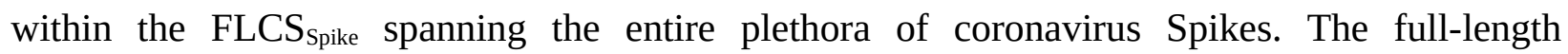
sequences were then run in the PrDos web-server [102] - which combines local sequence information and homology templates using iterative (psi) BLAST. Since the loop-disorder is highly contextual to its neighboring / flanking sequences and to that of the 'highly conserved' trimeric Spike structures (C ${ }^{\alpha}$-RMSD: $2.3 \AA$ ), the default setting of 'template-based' prediction (with the PrDos-FPR ${ }^{\ddagger 7}$ set to $5 \%$ ) was retained as 'turned on’. For all representative full-length

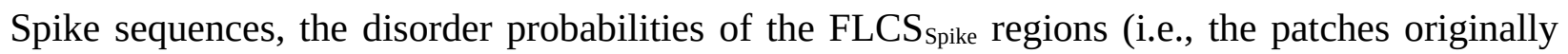
missing in the corresponding experimental structures) were unanimously found to increase sharply in the $\mathrm{N} \rightarrow \mathrm{C}$ direction around the pentapeptide motifs trending to local maximums (Figure 1). The regions, consequently mapped to the ascending halves of the corresponding curve-humps (Figure 1.A) - which should effectively mean 'growing disorders' associated with

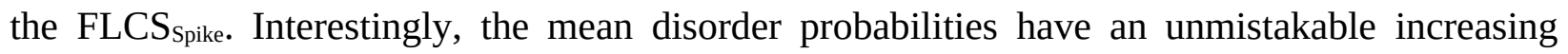
trend ( 0.45 for PSGAG $\rightarrow \rightarrow 0.55$ for PRARR) with the successive gradual incorporation of arginines in the pentapeptide sequence motif.

\subsection{Filling up the voids in the Spike structures: the FLCS $S_{\text {spike }}$ disordered ensembles}

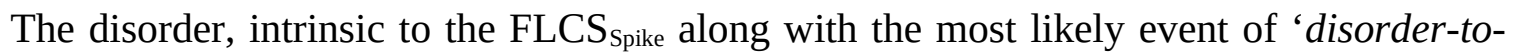
order transition' upon binding to Furin (see Introduction) makes the Spike-Furin iteration an interesting mechanistic chapter both in the context of coronavirus evolution and also in the general framework of proteolytic cleavages [41-43]. Particularly intriguing (and, counterintuitive almost) is the fact that the bait the SARS-CoV-2 Spike uses to attract their specific and dedicated host-proteases (e.g., Furin for SARS-CoV-2 Spike) are themselves structurally highly nonspecific or conformationally varied by virtue of their intrinsic disorder. Again, in the bound

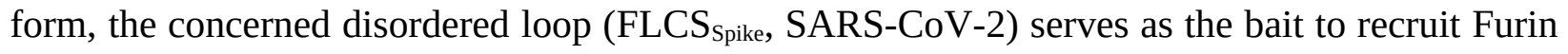
subsequent to which it needs to be jammed into a much restricted set of conformations for its efficient (proteolytic) cleavage [32]. The collective dynamics of the Spike-Furin interaction (SARS-CoV-2) would thus naturally lead to a conformational selection of the disordered FLCS $_{\text {Spike }}$ in its Furin-bound state. To sustain such an energetically costly 'conformational selection', an energy-source is hence required to compensate for such a high entropy-loss of the

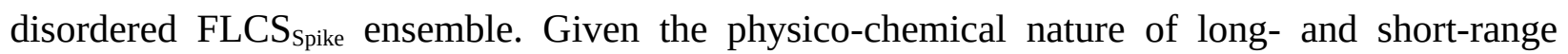
forces sustaining the native bio-molecular environment, such compensating energies would naturally be enthalpic in nature. Thus, an enthalpy - entropy compensation seems necessary. So, the obvious question that follows next would be as to what might be the source of this enthalpic energy to compensate for such a high entropy-loss?

7 FPR: False Positive Rate 
To address this, first and foremost, the missing disordered stretches in the representative SARSCoV-2 Spike pre-fusion structure (PDB ID: 6XR8) were ensemble-modeled (see, section 2.2, Materials and Methods) using its full-length amino acid sequence, derived from proteomic

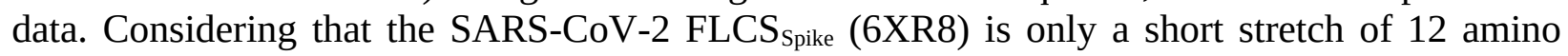
acid residues ( ${ }_{677}$ QTNSPRRARSVA $_{689}$ ), 500 uniquely varied loop-conformations were sampled for the missing patch, eventually leading to that many 'all-atom' trimeric SARS-CoV-2 Spike atomic models. Such an ensemble can essentially and adequately represent the conformational

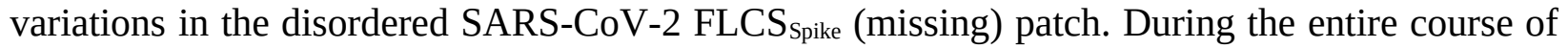
this modeling, the atoms that were already experimentally solved were retained as they were. The average $\mathrm{C}^{\alpha}$-RMS deviations, upon aligning the models pairwise in PyMol (https://pymol.org/ 2/), were found to be appreciably higher (4.71 $\AA$; SD: 0.82$)$ even for the short (101 atoms)

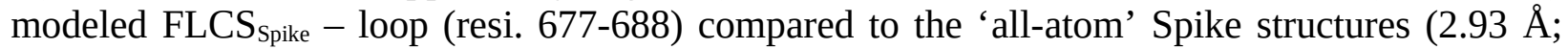
SD: 0.54 for 26940 aligned atoms). This further confirmed the disorder, intrinsic to the FLCS Spike $_{\text {; }}$ in SARS-CoV-2. To serve as a baseline, a similar approach was adapted to model the homologous missing patch $\left({ }_{664} \mathrm{SLLRSTS}_{670}\right)$ in SARS-CoV Spike (see, section 2.2, Materials and Methods) that was originally missing in the representative experimental structure (PDB ID: 7AKJ), albeit with a lower degree of conformational sampling proportional to a lower degree of

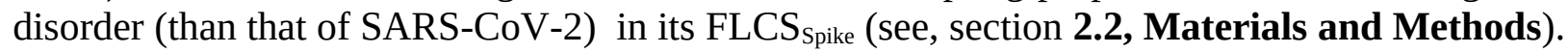

\subsection{Docking Furin onto Spike: using the pentapeptide activation loop to filter and accumulate correctly docked poses}

Once the (unbound) disordered FLCS $_{\text {Spike }}$ ensemble (SARS-CoV-2) was made ready (section 3.3), a series of blind docking experiments was conducted in ClusPro 2.0 [65] (see 2.3.1, Materials and Methods), wherein Furin (ligand) was docked ab-initio onto each of the 500 Spike 'all-atom' atomic models (receptors) without imposing any additional 'active-site / contact residue' constraints. This resulted in an initial pool of 53215 Spike-Furin unsorted docked poses (Figure S3, Supplementary Materials) with the ligand docked at widely varying sites spread all over the trimeric Spike receptor. The extra-large sample space of returned docked poses (of the order of fifty thousands) ensured necessary and sufficient coverage of the ligand - receptor orientational space in docking while the detailed iterative blind docking protocol (Cluspro 2.0) maintained the fine-grain qualities of the docked poses. All docked poses for each ensembledocked set (consisting of 500 receptor templates unique for their $\mathrm{FLCS}_{\text {spike }}$ ) were then

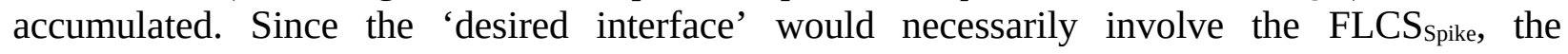
pentapeptide motif $\left({ }_{681} \mathrm{PRRAR}_{685}\right.$ ) was used as 'contact residue filters' to discard obviously and/or trivially incorrect docked poses. To that end, buried surface areas (BSA) (see section 2.3.2.1, Materials and Methods) were computed for all residues in each docked pose and the interfacial residues (BSA $\neq 0$, see section 2.8) were identified. In addition, the BSA values for

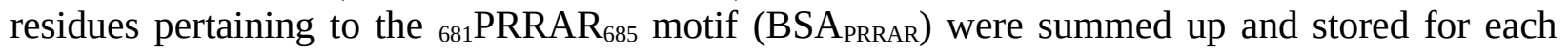
docked pose. If the interfacial residues, so identified, happen to contain any fraction of the pentapeptide motif (i.e., BSA $\mathrm{PRRAR}>0$ ), the docked pose was considered plausible and worthy to be carried for the next round (i.e., filtered in). All three chains of the Spike - trimer (harboring this FLCS $_{\text {Spike }}$ ) were treated equally likely to serve as the docking site. This simple technique ensured that all filtered docked poses (7184 of them) have the desired Spike-Furin interface harboring the FLCS $_{\text {Spike }}$ [48]. It was interesting to note that in the top-ranked docked poses 
(ranked by Cluspro's internal score) for almost all ensemble-docked batches (in 497 out of 500 cases) Furin had an unmistakable preference to dock at the FLCS $_{\text {Spike }}$ (i.e., BSA PRRAR $>0$ ) even with this unbiased $a b$-initio blind docking protocol.

After the initial filtering of 'plausible' docked poses (those that harbors the FLCS Spike $_{\text {at }}$ the Furin - Spike interface), $\mathrm{Sc}^{\mathrm{FLCS}}$ (see section 2.3.2.2, Materials and Methods) was computed for the BSA - filtered interfaces alone. To render a discerning scoring function appropriately combining both surface fit and overlap at the interface, BSA PRRAR was further normalized to nBSA PRRAR (see section 2.3.2.1, Materials and Methods). A second subsequent filtering was performed on the filtered set wherein nominal relaxed cutoffs (slightly more restraint than merely non-zero) on nBSA $_{\text {PRRAR }}$ and $\mathrm{Sc}^{\mathrm{FLCS}}$ were jointly implemented (nBSA $\mathrm{PRRAR}>0.05, \mathrm{Sc}^{\mathrm{FLCS}}>0.2$ ) based on their general trends [71,77]. In effect, each docked pose had to attain minimum thresholds in terms of both the goodness of fit of the interacting surfaces and their extent of conjointness. The second round of filtering thus resulted in more realistic poses and further reduced their number from 7184 to 5735 . These poses were then scored and re-ranked by an appropriately weighted scoring function $\left(\mathrm{S}_{\text {dock }}\right)$ combining $\mathrm{nBSA}$ and $\mathrm{Sc}$ (see section 2.3.2.3, Materials and Methods). The reranking ensured careful and combined monitoring of both the geometric fit of the interacting surface patches at the interface and also the extent of that fit. The average $\left\{<\right.$ nBSA $\left.A_{\text {PRRAR }}\right\rangle$, $\left\langle\mathrm{Sc}^{\mathrm{FLCS}}>\right\}$ values for the top 10 (re-)ranked docked poses (Table S2, Supplementary Materials) were suggestive of their elevated surface fit $\left(\left\langle\mathrm{Sc}^{\mathrm{FLCS}}\right\rangle: 0.70, \mathrm{SD}^{8}: 0.04\right)$ and decent surface overlap ( $<$ nBSA $_{\text {PRRAR }}>$ : 0.211, SD: 0.034) at the interface. The same averages for the top 100 (re-)ranked docked poses were 0.66 (SD: 0.06), 0.129 (SD: 0.053) for Sc ${ }^{\mathrm{FLCS}}$, nBSA ${ }_{\text {PRRAR }}$ (Table S2, Supplementary Materials) - which fall very well within the range of values obtained for the same or equivalent parameters surveyed at native protein interiors [72-74] as well as at native/near-native protein interfaces [71,77]. Top ranked docked poses were all visually surveyed and scrutinized in PyMol. No noticeable inconsistencies were observed. The top ranked docked pose $\left(\mathrm{RR} 1_{\mathrm{CoV}-2}\right)$ with a $\mathrm{S}_{\text {dock }}$ score of 0.972 was also ranked first in terms of surface

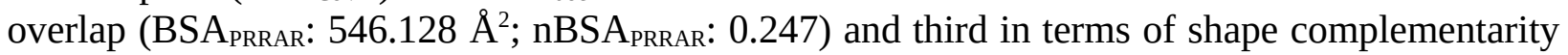
(Sc ${ }^{\mathrm{FLCS}}$ : 0.77$)$ to be attained at the Spike-Furin interface. $\mathrm{RR} 1_{\mathrm{CoV}-2}$ was then taken to energy minimization cycles followed by a (sufficiently long) explicit water, all atom molecular dynamic simulation (see, section 2.4, Materials and Methods) to account for the structural dynamics of the Spike-Furin interaction in SARS-CoV-2.

In contrast, a guided docking approach using 'Zdock + IRaPPA re-ranking' (see sections 2.3.1.2, 2.3.1.3, Materials and Methods) was directly adapted in parallel as a mean to cross-validate the results obtained from $\mathrm{RR} 1_{\mathrm{CoV}-2}$ as well as for the baseline subject in SARS-CoV leading to two more top-ranked models, $\mathrm{ZR} 1_{\mathrm{CoV}-2}$, $\mathrm{ZR} 1_{\mathrm{Cov}}$ respectively - both of which were then undertaken long (100 ns) MD simulation runs, subsequent to equivalent rounds of energy minimization.

\subsection{Plausible 'disorder-to-order' transition triggered by salt-bridge dynamics at the Spike- Furin interface: the 'Salt-bridge hypothesis'}

One common intuition to jam the high-entropy FLCS $_{\text {spike }}$ disordered loop (SARS-CoV-2) into the Furin bound enzyme-substrate complex would be to stabilize the highly localized

8 SD: Standard Deviation 
positive charge cloud electrostatically. It is rather well known that electrostatic interactions play an important role in the dynamic sustenance and transitions associated with protein disorder

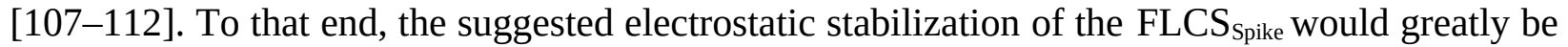
benefited by the structural proximity of oppositely charged (i.e., anionic) amino acids (coming from Furin) by triggering the formation of Spike-Furin interfacial salt-bridges. The plausibility of the 'salt-bridge hypothesis' is further enhanced by the presence of a surface groove around the

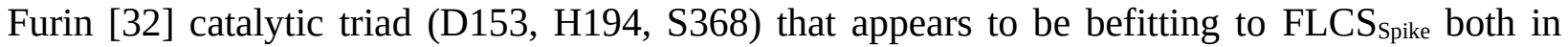
terms of shape and electrostatics (Figure 2). This groove serves as a potentially attractive

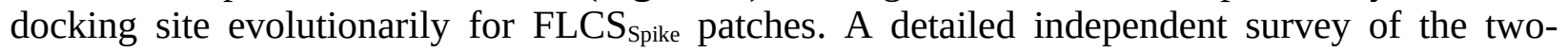
domain Furin structure (PDB ID: 1P8J, chain A, see section 2.1.1, Materials and Methods) further reveals that it has an abundance of anionic residues (30 aspartates and 25 glutamates) which are spread rather homogeneously all over its catalytic and P domains (see Introduction). While the majority (58\%) of these residues are either partially or completely exposed to the solvent (bur $\geq 0.05$ ) (see section 2.3.2.1, Materials and Methods), those that are part of the catalytic domain (Figure 2) are of interest to the given context. Note that even partially exposed anionic residues with lesser exposure (say, $0.05<$ bur $\leq 0.15$ ) in vicinity of the triad can, in principle, seldom flip into a more extended and exposed conformation during the course of the Spike-Furin binding (till the Spike-S1/S2 cleavage). Hence, these may also potentially contribute to stabilize the proposed dynamically interchangeable ionic bond networks at the Spike-Furin interface (SARS-CoV-2). To have a closer look into these 'residues of interest', first, a subset of anionic residues in Furin was assembled, where at least one heavy side chain atom from each residue is located (in its crystalline equilibrium state) within a sphere of radius $20 \AA$ centered at the side chain centroid of the Furin catalytic triad (D153, H194, S368). This subset contained 25 residues, most of which (18 out of 25) are 'partially exposed' or 'exposed' (Table S3, Supplementary Materials) and hence should be amenable and approachable for formation of interfacial salt-bridges. Again, some of these partially or completely exposed anionic residues (viz., D191, D228, E236, E257, D258, D259, D355, D306; see Table S3, Supplementary Materials) are rather proximal to the catalytic triad (within $15 \AA$ from its side chain centroid). Together, these constitute a non-rigid set of anionic Furin residues that

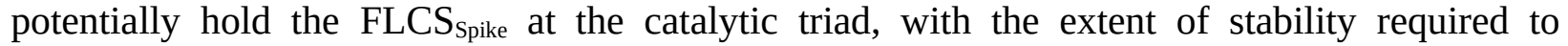
encompass the cleavage, by virtue of dynamically engaging themselves into ionic bond interactions with the $\mathrm{FLCS}_{\text {Spike}}$-arginines coming from ${ }_{681} \mathrm{PRRAR}_{685}$. The positioning of these anionic side chains also appears to be strategic (Figure 2) such that cumulatively the salt-bridges almost never get dissolved during the entire course of the Spike-Furin binding. Such dynamic networks of potentially interchangeable ionic bonds could also be a prime source for the enthalpy required to compensate for the presumably high entropic loss of the FLCS spike $_{\text {upon binding to }}$ Furin. The process would also necessarily accompany a concomitant transition of the FLCS Spike from a disordered (unbound) to a relatively ordered (Furin bound) state.

\subsection{Validations and cross-validations of the 'salt-bridge hypothesis'}

3.6.1. In $R R 1_{\mathrm{CoV}-2}$

In order to test the validity of the 'salt-bridge hypothesis', an all-atom explicit water MD simulation (of 300 ns) was run (see section 2.4, Materials and Methods) for the top ranked Spike-Furin docked pose $\left(\mathrm{RR} 1_{\mathrm{CoV}-2}\right)$, and, the trajectories were tested for the presence of salt- 
bridges (see section 2.5.1, Materials and Methods). Prior to the production run, the structural template $\left(\mathrm{RR} 1_{\mathrm{Cov}-2}\right)$ was undertaken rigorous rounds of energy-minimization with temperature equilibration (see section 2.4, Materials and Methods). Structural snapshots were sampled at 10 ps (regular) intervals leading to 30000 snapshots (or time-stamps) accounting for the $300 \mathrm{~ns} \mathrm{long}$ MD simulation trajectory and salt-bridges were extracted from each snapshot from its interfacial contact map (see section 2.5.1, Materials and Methods). To set a reference, ionic bond networks were also surveyed in the pool of 100 top ranked static docked poses (Table S2, Supplementary Materials). In both ensembles, dynamic and static, the identification of saltbridges were followed by their individual survey and also a statistical analyses of dynamic or static 'ensemble descriptors of salt-bridges' (e.g., persistence/occurrence and average contact intensities; see section 2.5.2, Materials and Methods). Together these parameters can effectively be used to decipher and interpret the complex nature of salt-bridge dynamics associated with protein disorder transitions [49,50]. One of the trademark features of salt-bridge dynamics associated with disorder transitions is a trade-off or an optimal balance between transience and persistence of salt-bridges which serves to sustain a disordered state while a shift of this balance leads to disorder transitions [49,50].

To that end, explicit lists of ionic bond interactions independently for the dynamic (Table 1), static ensembles (Table S4, Supplementary Materials) were sorted based on their persistence (dynamic), occurrence (static) (see section 2.5.2.2., Materials and Methods). Given that the static ensemble consisted of near-native as well as less near-native poses (Figure S4, Supplementary Materials), it was interesting to find that all (or almost all) Spike-Furin interfacial salt-bridges that had occurred (at least once) in the static ensemble were also found (at least ephemerally) in the dynamic ensemble. The persistence/occurrence profiles collectively reveal that the Spike-Furin interaction has a preferential set of ionic bonds in terms of forming dynamically interchangeable salt-bridges - which may vary in their occurrence among the plausible docked poses. Since, RR $1_{\mathrm{Cov}-2}$ presents possibly the most preferred conformation of the FLCS $_{\text {spike }}$ in its Furin bound state (in SARS-CoV-2), the dynamically sustained high persistence arginine - salt-bridges (in ${ }_{681} \mathrm{PRRAR}_{685}$ ) found in $\mathrm{RR} 1_{\mathrm{CoV}-2}$ are the most plausible, frequently forming Spike-Furin interfacial salt-bridges (Figure S4, Supplementary Materials) among alternatives.

The first noticeable observation in the dynamic ensemble (pertaining to $R R 1_{\mathrm{CoV}-2}$ ) was that the three arginines (R682, R683, R685) in the ${ }_{681}$ PRRAR $_{685}$ almost always remain engaged in dynamically interchangeable ionic bonds formed with anionic side chains coming from the host Furin. These amenable anionic side chains (D191, E230, D233, E236, D259, D264, D306, see Table S4, Supplementary Materials) are indeed physically proximal to the catalytic triad

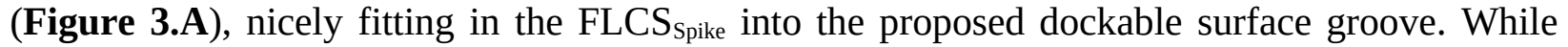
most (if not all) of them fall into the non-rigid 'expected' set (Table S3, Supplementary Materials), they should collectively present some correlated movements with FLCS Spike $_{\text {in order }}$ to remain conformationally viable for dynamically stable ionic bond networks. Further, as reflected from the distribution of salt-bridge persistence (see Table 1) the three arginines in ${ }_{681}$ PRRAR $_{685}$ were often involved in multiple and interchangeable ionic bonds. In other words, the same arginine (target) in ${ }_{681} \mathrm{PRRAR}_{685}$ can simultaneously be in contact (at a given instance) 
with more than one negatively charged residues coming from the host Furin (neighbor). This will lead to the formation of non-disjoint sets of target-neighbor ionic bond pairs (i.e., salt-bridges) for the same target. In other words, sets of salt-bridges having distinct anionic partners for a given target arginine ( ${ }_{681} \mathrm{PRRAR}_{685}$ ) would thus be non-disjoint. This implies that the persistence values of the arginine - salt-bridges (in ${ }_{681} \mathrm{PRRAR}_{685}$ ) may add up to more than unity (see Table 1). In fact, dynamic interchangeability of counter-ions are common characteristic features of saltbridges formed at disordered protein regions and/or [49,50] disorder-globular interfaces [86,110] wherein the formed ion pairs keep changing their counter-ionic partners [49]. Such collective dynamics results in salt-bridges of varying persistence and multiplicity across the trajectory, including persistent as well as transient salt-bridges [49,50,109,111]. Transient and/or unfavorable salt-bridges have been revealed to be functionally optimized in proteins $[49,112]$ and are often found on enzyme surfaces [112] as well as on strategic locations sparsed around extended disordered protein regions [49]. For the later case, one of the evolved key mechanisms towards achieving this functional optimization is to make their charged side chains often amenable to proximally approached ordered protein interactors. The transient nature facilitates the exchange of their counter-ionic partners, thereby triggering the switch from intra- to intermolecular (interfacial) salt-bridges. The multiplicity (or promiscuity [49]) in the choice of the counter-ionic partner in a salt-bridge can further be chemically and electrochemically rationalized from the bifurcated nature of side chain groups with degenerate charge centers in four out of the five charged amino acids (guanidium ${ }^{+}$: Arg; carboxylate: Asp, Glu; imidazol ${ }^{+}$: His + ) in proteins. However, in spite of this observed multiplicity, each of the three arginines in ${ }_{681}$ PRRAR $_{685}$ seemed to have their own preference for particular anionic partners: E230 for R682 (pers: 0.93), E236 for R683 (pers: 0.85) and D306 for R685 (pers: 0.90) (see Figure 3.C). These three key anionic residues involved in the highest persistent arginine - salt-bridges (in ${ }_{681}$ PRRAR $_{685}$ ) was further envisaged to form a combined molecular entity (let's call it the 'anionic triad') made up of discrete molecular components (anionic side chains). The anionic triad and the catalytic triad (D153, H194, S368) remained proximal and approachable (average centroid-to-centroid distance between side chain atoms: $13.16 \AA$, SD: 0.72 ) throughout the 300 ns trajectory $\left(\mathrm{RR} 1_{\mathrm{CoV}-2}\right)$ with the display of correlated movements as if like an ordered pair (Figure 4.A). To analytically confirm the visually apparent correlated movement, the inter-triad angle $(\varepsilon)$, defined as the planer internal angle subtended by the two position vectors originated from the Furin molecular centroid that connect the centroids of the two triads (Figure 4.B), was surveyed across the trajectory. The inter-triad angle was found to be strictly constrained $\left(<\varepsilon>=45.1^{\circ}, \mathrm{SD}=4.7\right.$ ) having an apparently bi-modal distribution with two modes at $\sim 42.1^{\circ}$ and $\sim 52.8^{\circ}$.

Other interfacial salt-bridges barring those involving the three arginines in ${ }_{681} \mathrm{PRRAR}_{685}$ $\left(\right.$ FLCS $\left._{\text {Spike }}\right)$ was also surveyed in the same details. Among these, one salt-bridge, 'E654 $4_{\text {sike }} \leftrightarrow$ R193 ${ }_{\text {Furin }}$, (see Table 1) was noticeable both in terms of its high persistence (pers: 0.65) and the opposite trend in the distribution of its charge centers (negative in Spike and positive in Furin, for a change) in contrast to the arginine - salt-bridges (in ${ }_{681} \mathrm{PRRAR}_{685}$ ). Other salt-bridges with brief/instantaneous occurrences (pers $<0.1$ ) could be considered ephemeral. The collective interplay of these fleeting salt-bridges triggers a 'transient dynamics' in disordered protein regions that is indispensable in retaining their flexibility [49] and is also pivotal towards 
imparting a critical behavior in associated disorder transitions among multiple self-similar fractal states [50]. The presence of transient salt-bridges (pers<0.1) in significant fractions $(70.5 \%$ in the dynamic ensemble of $\mathrm{RR} 1_{\mathrm{CoV}-2}$, see Table 1) signals for relatively ordered metastable Furinbound states of the FLCS Spike which together retain enough flexibility (see Video S1, Supplementary Materials) to favor the Spike-S1/S2 proteolytic cleavage [33,35].

\subsubsection{In $\mathrm{ZR} 1_{\mathrm{CoV}-2}$}

As a cross-validation of the 'salt-bridge hypothesis', an independent guided docking was performed in Zdock using IRaPPA re-ranking (see section 2.3.1.2, Materials and Methods) and the returned top ranked docked pose $\left(\mathrm{ZR} 1_{\mathrm{CoV}-2}\right)$ was simulated in yet another independent $\mathrm{MD}$ simulation run for 100 ns (see section 2.4, Materials and Methods). Following on, structural snapshots were sampled at $10 \mathrm{ps}$ intervals (likewise to that of $\mathrm{RR} 1_{\mathrm{CoV}-2}$ ) leading to 10000 snapshots. Salt-bridges were then extracted from each snapshot from its interfacial contact map (see section 2.5.1, Materials and Methods) and further sorted based on their dynamic persistence. A second sorted list, equivalent to that of $\mathrm{RR} 1_{\mathrm{CoV}-2}$ (Table 1), was procured for ZR1 $1_{\mathrm{CoV}-2}$ (Table S5, Supplementary Materials). Counter-ionic partners in most high persistence arginine - salt-bridges (in ${ }_{681} \mathrm{PRRAR}_{685}$ ) were preserved in both (dynamic) ensembles ( $\left.R R 1_{\mathrm{CoV}-2}, \mathrm{ZR} 1_{\mathrm{CoV}-2}\right)$ pairing either with the same $\left(\mathrm{R} 682_{\text {Spike }} \leftrightarrow \mathrm{E} 230_{\text {Furin }}\right.$, pers: 0.93, 0.99 respectively) or altered partners (R683 ${ }_{\text {Spike }} \leftrightarrow$ E236 $6_{\text {Furin }}$, pers: 0.85 in RR1 $1_{\text {CoV-2}}$; R685 $5_{\text {Spike }}$

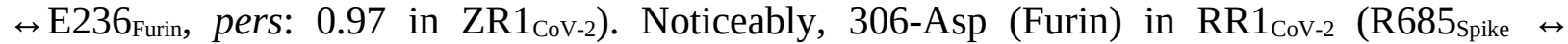
D306 Furin, pers: 0.90) was replaced by 259-Asp (Furin) in ZR1 $1_{\mathrm{CoV}-2}\left(\mathrm{R} 683_{\text {Spike }} \leftrightarrow \mathrm{D} 259_{\text {Furin, }}\right.$ pers: 0.49). This suggests that there might be multiple plausible conformations (docked poses) mapping to unique (i.e., non-degenerate) yet befitting ionic bond network archetypes - all of which could enable the Spike-Furin binding. In fact to have such essential and nominal degrees of freedom generally in bio-molecular fitting (including self-fitting or folding) allows the system to breathe and is of no great surprise (at least) in protein science, often directed by satisfying optimized global physico-chemical constraints while retaining their structural degeneracy. The revelation of secondary and super-secondary structural motifs [113], packing motifs within native globular protein interiors [73], composite salt-bridge motifs within proteins and protein complexes [86] as well as the plausibility of the partly proven idea of alternative packing modes potentially leading to the same native protein fold (or a befitting hydrophobic core) $[114,115]$ each and all are instances of the phenomena.

The Spike-Furin interfacial salt-bridges (in both $\mathrm{RR} 1_{\mathrm{CoV}-2}$ and $\mathrm{ZR} 1_{\mathrm{CoV}-2}$ ) generally varied in terms of their contact intensities (CI) (see section 2.5.2.2, Materials and Methods) while the high persistence salt-bridges (formed near the Furin catalytic triad) were by and large, densely connected throughout their entire simulation runs (Figure 5), hitting appreciably high ACI values in most cases (Table 1, Table S5). Most high persistence salt-bridges also frequently retained maximally connected $\left(\mathrm{CI}=\mathrm{CI}_{\max }=4\right)$ closed ionic bond (bipartite) motifs between bifurcated oppositely charged side chain groups in both subjects (Figure 5, insets).

$\mathrm{RR} 1_{\mathrm{CoV}-2}$ and $\mathrm{ZR} 1_{\mathrm{CoV}-2}$ also had great resemblance in their frequency distribution profiles for the Spike-Furin interfacial salt-bridge persistence(s) (unweighted as well as weighted: see section 2.5.2.2, Materials and Methods) [49] (Figure S5, Supplementary Materials). To quantify this 
resemblance, the entire theoretical range of persistence (pers) [0,1] was partitioned into 20 equally spaced bins, and for each ensemble, the normalized frequencies of salt-bridges falling within each persistence-bin (of bin-width: 0.05) were computed. A similar approach was adapted for weighted persistence (wpers) (see section 2.5.2.2, Materials and Methods) which maps to a theoretical range of $[0,4]$, equaling a bin-width of 0.2 for a 20-bin model. The Pearson Correlation between these obtained normalized frequencies from the two ensembles (RR $1_{\mathrm{Cov}-2}$, $\mathrm{ZR} 1_{\mathrm{CoV}-2}$ ) was found to be 0.97 for persistence (Figure S5.A) and 0.93 for weighted persistence (Figure S5.B) (P-value $<0.00001$ for both which is significant at $99.9 \%$ level). The same correlation (Pearson's) was found to be 0.66 (P-value: 0.001445, significant at 99.9\% level) between the frequency distribution profiles $\left(\mathrm{RR} 1_{\mathrm{CoV}-2}, \mathrm{ZR} 1_{\mathrm{CoV}-2}\right)$ which are plotted for average contact intensities (ACI) of ionic bonds formed at the Spike-Furin interface (Figure S5.A, inner set, Supplementary Materials).

\subsubsection{In $\mathrm{ZR1} 1_{\mathrm{CoV}}$, the baseline}

As introduced in section 2.3.1.3 (Materials and Methods), $\mathrm{ZR} 1_{\mathrm{CoV}}$ (the representative Spike-Furin interaction in SARS-CoV, 2002/2003) served as the baseline for the Spike-Furin interaction in SARS-CoV-2. As has been already discussed (see section 2.2, Materials and Methods), the $\mathrm{FLCS}_{\text {Spike }}$ patch in $\mathrm{ZR} 1_{\mathrm{Cov}}$ (664 $\mathrm{SLLRSTS}_{670}$, originally missing in 7AKJ) is much shorter than its homologous missing patch in 6XR8 ( ${ }_{677}$ QTNSPRRARSVA 689 ) mapping to their corresponding degrees of disorder (higher in the later). The relative composition of the two patches and their pairwise alignments (Figure S1, Supplementary Materials) further support the observation that indeed a lesser degree of disorder is expected for the concerned patch in SARS-CoV than that in SARS-CoV-2. Most notably, the third arginine (R685) of ${ }_{681}$ PRRAR $_{685}$ in CoV-2 FLCS Spike $_{\text {is evolutionarily conserved (as R667) also in CoV FLCS }}$ Spike (see section 2.2) (Figure S1, Supplementary Materials). A closer look into the pairwise alignments of the two sequences (Figure S1, Supplementary Materials) also reveals the strategic insertion of a dipeptide unit $\left({ }_{681} \mathrm{PR}_{682}\right)$ followed by two non-synonymous replacements (L665 $\rightarrow \mathrm{R} 683$, L666 $\rightarrow$ A684) in the disordered activation loop (see Introduction) of the CoV-2 Spike (6XR8) with respect to its ancestral homologous Spike in CoV (7AKJ). With this background, when we had a good look at the MD simulation trajectories of $\mathrm{ZR} 1_{\mathrm{Cov}}$ we found something very insightful. The conserved arginine (R667) in 7AKJ was found to cover much space in ZR1 $1_{\text {Cov }}$ throughout its entire dynamic trajectory (100 ns) together with an accompanying nearby lysine (K672), feeling up for the absence of the other arginines (in reference to ${ }_{681} \mathrm{PRRAR}_{685}, \mathrm{CoV}-2$ ) leading to the formation of a homologous dynamically persistent network of interfacial salt-bridges in CoV. The conserved arginine, R667 alone seemed to engage as many as three counter-ionic Furin side chains (E230, E257, D258) forming two high persistent (pers: 0.62, 0.95) and one moderately persistent (pers: 0.28) salt-bridges (see Table S6, Supplementary Materials), while the neighboring lysine (K672) was found in pair with D258 (Furin) with a persistence of 0.58. Notably, D258 among the Furin anionic residues, shared persistent salt-bridges simultaneously with K672 and R667 (see Table S6, Supplementary Materials). In contrast to the ${ }_{681}$ PRRAR $_{685}$ (CoV-2 Spike), here, in context to ${ }_{664}$ SLLRSTS $_{670}$ (CoV Spike), the absence of the long and electrostatically repelling neighboring arginine side chains offers R667 the physical space to remain substantially flexible to be simultaneously involved in multiple high persistence saltbridges. The formation of these interfacial salt-bridges are favored by the proximal looping of 
the flanking lysine (see Figure S6, Supplementary Materials). The two non-adjacent basic residues (R667, K672) together serves to sustain the homologous Spike-Furin interface in CoV, also, by the formation of several dynamically persistent ionic bonds. To that end, if the emergence of the 'PRRAR' motif (in CoV-2 Spike) is to be considered a solution that is optimized for the most efficient Spike-S1/S2 cleavage at the Spike-Furin interface, the interplay of R667 and K672 in context to the homologous FLCS Spike $_{\text {in }} \mathrm{CoV}$ appears to be analogous to the event of structural relaxation in mutant protein cores. In other words, the way R667 and K672 cover up the physical space in SARS-CoV to sustain the Furin and catalyze the Spike-S1/S2 cleavage appear to resemble with the collective conformational readjustments of neighboring residues, filling up for packing defects and/or cavities/holes introduced upon hydrophobic substitutions/truncation in native protein core(s) $[116,117]$. Having said that, the total number of non-redundant Spike-Furin interfacial salt-bridges were found to be literally doubled by the incorporation of the additional arginines (PRRAR) in CoV-2 (17: RR1 $1_{\mathrm{CoV}-2}, 20$ : ZR1 $1_{\mathrm{CoV}-2}$ ) compared to the baseline (9: $\mathrm{ZR} 1_{\mathrm{Cov}}$ ) in $\mathrm{CoV}$. Also, the ionic bond networks seemed to be certainly more dense and intense in CoV-2 with a CCI (see section 2.5.2.2, Materials and Methods) of 7.65, 10.16 in $\mathrm{RR} 1_{\mathrm{CoV}-2}, \mathrm{ZR} 1_{\mathrm{CoV}-2}$ compared to 5.48 in $\mathrm{CoV}\left(\mathrm{ZR} 1_{\mathrm{CoV}}\right)$. These are signatures of the proposed optimization characteristic of 'gain of function' mutational studies (see Introduction).

\subsection{Enthalpy - entropy compensation involved in the Spike-Furin interaction}

The 'salt-bridge hypothesis' (see section 3.5) was proposed based on the intuition that the

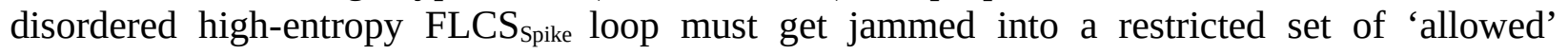
conformations into Furin that are favorable for the Spike-S1/S2 cleavage. Such a conformational selection should necessarily accompany electrostatic stabilization of the highly localized positive charge cloud on ${ }_{681} \mathrm{PRRAR}_{685}$ (FLCS $_{\text {Spike}}$ ). The Spike-Furin interaction thus implicitly speaks in favor of a 'disorder-to-order transition' that needs an enthalpic source to compensate for the high entropic cost (loss) intrinsic to the supposed transition. One prime source for such enthalpic compensation are salt-bridges for they impart local rigidity in proteins by jamming conformations [86]. To that end, the 'salt-bridge hypothesis' was only found more plausible by the detection of a potentially dockable surface groove to fit in the FLCS $S_{\text {spike }}$ near the Furin catalytic triad (see section 3.5), surrounded by exposed anionic residues that seemingly have the potential to the form salt-bridges with the $\mathrm{FLCS}_{\text {Spike }}-$ arginines (in ${ }_{681} \mathrm{PRRAR}_{685}$ ). All structural dynamics analyses (see section 3.6) unanimously and collectively reveal that the FLCS $_{\text {Spike }}$ fits nicely and stably into the proposed dockable groove, stabilized by the formation and sustenance of dynamically interchangeable interfacial salt-bridges (validated in $\mathrm{RR} 1_{\mathrm{Cov}-2}$, and, crossvalidated in $\left.\mathrm{ZR} 1_{\mathrm{CoV}-2}\right)$. Together these results speak in favor of an 'enthalpy entropy compensation' intrinsic to the transition from the disordered (free) to the relatively ordered

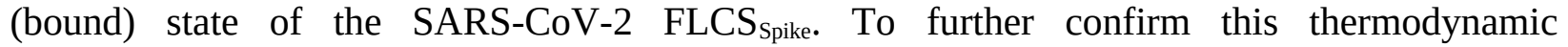
phenomenon, we computed actual structure - based all atom thermodynamic parameters by FoldX (see section 2.6, Materials and Methods) for the respective MD simulation trajectories pertaining to $\mathrm{RR} 1_{\mathrm{CoV}-2}, \mathrm{ZR} 1_{\mathrm{CoV}-2}(300 \mathrm{~ns}, 100 \mathrm{~ns}$ ) and compared the relevant transition enthalpic $\left(\Delta \mathrm{H}_{\mathrm{vdw}}, \Delta \mathrm{H}_{\text {elec }}\right)$ and entropic $\left(\Delta \mathrm{S}_{\mathrm{mc}}, \Delta \mathrm{S}_{\mathrm{sc}}\right)$ terms associated with the Spike-Furin binding / complexation. Both molecules in their integral forms were considered for the FoldX energy calculations. To set up an appropriate baseline, $\mathrm{ZR} 1_{\mathrm{Cov}}$ was also included in the calculations and 
comparison. All relevant transition enthalpic and transition entropic terms individually as well as collectively had retained a counter trend $\left(\Delta \mathrm{H}_{\mathrm{vdw} / \mathrm{elec}}<0, \Delta \mathrm{S}_{\mathrm{mc} / \mathrm{sc}}>0\right)$ throughout the entire trajectories of all subjects, $\mathrm{RR} 1_{\mathrm{CoV}-2}, \mathrm{ZR} 1_{\mathrm{CoV}-2}$, as well as the baseline, $\mathrm{ZR} 1_{\mathrm{CoV}}$ (Table 2, Figure 6). This is suggestive of enthalpy - entropy compensations accompanying both Spike-Furin binding events (in CoV-2, CoV). However, as is reflected from the relative magnitudes of the $\Delta \mathrm{H}, \Delta \mathrm{S}$ terms (Table 2, Figure 6), the binding in $\mathrm{CoV}-2\left(\mathrm{RR} 1_{\mathrm{CoV}-2}, \mathrm{ZR} 1_{\mathrm{CoV}-2}\right)$ is attributed with higher entropic costs of the event and therefore with a concomitant higher degree of enthalpic compensation than that in $\mathrm{CoV}\left(\mathrm{ZR} 1_{\mathrm{CoV}}\right)$.

As a second test, the individual main chain and side chain conformational entropies for the receptor (Spike) and the ligand (Furin) were also surveyed in $\mathrm{RR} 1_{\mathrm{CoV}-2}, \mathrm{ZR} 1_{\mathrm{CoV}-2}, \mathrm{ZR} 1_{\mathrm{CoV}}$ (throughout their respective trajectories) and compared between their unbound ( $\Delta S_{m c}^{\text {receptor } / \text { ligand }}$ ) and bound ( $\Delta S_{m c / s c}^{\text {complex }}$ ) states. Entropic terms derived independently from both binding partners (Spike and Furin) in their unbound states $\left(\Delta S_{m c}^{\text {receptor }}, \Delta S_{m c}^{\text {ligand }}, \Delta S_{s c}^{\text {receptor }}, \Delta S_{s c}^{\text {ligand }}\right.$; see section 2.6, Materials and Methods) were found to be fairly stable over time (Figure S7, Supplementary Materials) - all of which get drastically reduced $\left(\Delta S_{m c}^{\text {receptor } / l i g a n d}>>\right.$ $\Delta S_{m c / s c}^{\text {complex }}$; see Table 3) confirming the 'entropy arrest' (refer to section 2.6, Materials and Methods) implicit to the Spike-Furin complexation in both systems (CoV-2, CoV). Though, the comparative transition entropy profiles and the time-averages were in the same range of values for both systems, literally all the surveyed terms had a rise of about $3-5 \%$ in terms of their average trends from the former to the later complex (Table 3). Perhaps with no great surprise, the most prominent rise $(\mathrm{CoV} \rightarrow \mathrm{CoV}-2)$ was found for the side chain conformational entropies (5.7\%) of the receptor molecule ( $\Delta S_{s c}^{\text {receptor }}$, i.e., Spike) undergoing the transition, naturally for the sequence differences pertaining to the $\mathrm{FLCS}_{\text {Spike }}$ in both.

The binding free energy overall was mildly disfavored (i.e., $\Delta \mathrm{G}_{\text {binding }}$ mildly positive) in both Spike-Furin binding events (Table 2) suggesting perhaps to the characteristic formation of metastable and multi-stable interfaces throughout the coronavirus lineage. A strict negative $\Delta \mathrm{G}_{\text {binding }}$ was obtained in $17.1 \%, 21.5 \%$ of the time-frames in the CoV-2 trajectories: $\mathrm{RR} 1_{\mathrm{CoV}-2}$, $\mathrm{ZR} 1_{\mathrm{CoV}-2}$, while the same fraction was found to be merely $1.8 \%$ in $\mathrm{ZR} 1_{\mathrm{Cov}}$, the baseline (in CoV). The metastabilities (suggesting an 'on-and-off' mode of binding) appear to be of no great surprise and perhaps anticipated given that the Spike-Furin binding works like a preface to the cleavage of a desired peptide bond that seems to be favored upon the transient formation of certain energetically favorable intermediate conformations in the bound FLCS $S_{\text {Spike. }}$ In fact, given that such disordered activation loops (like that of FLCS $_{\text {Spike }}$ ) are known to serve as key structural and kinetic determinants of protease substrates [40], it would be worth exploring (via future studies) across other families of proteases (see Introduction) harboring such cleavage loops, as to whether the metastabilities also holds true in them. Apart from the revealed characteristic metastabilities, the comparative free energy values for the Spike-Furin binding were roughly twice as much in magnitude in $\mathrm{CoV}\left(<\Delta \mathrm{G}_{\text {binding }}>=6.429 \mathrm{kcal} \mathrm{mol}^{-1}, \mathrm{SD}=3.094: \mathrm{ZR} 1_{\mathrm{Cov}}\right)$ compared to those in CoV-2 $\left(<\Delta \mathrm{G}_{\text {binding }}>=3.687,3.043 \mathrm{kcal} \mathrm{mol}^{-1}, \mathrm{SD}=3.868,3.843: \mathrm{RR} 1_{\mathrm{CoV}-2}, \mathrm{ZR} 1_{\mathrm{CoV}-2}\right)$.

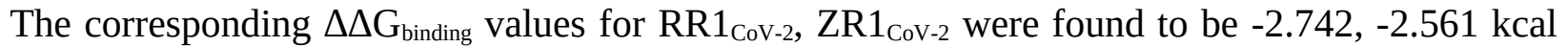
$\mathrm{mol}^{-1}$ (see section 2.6, Materials and Methods) - which speak directly in favor of a much more 
facilitated transition in the evolutionarily later event, signaling for the intended optimization (irrespective of whether natural or not) to have indeed occurred in SARS-CoV-2.

\subsection{Using the Ramachandran Plot to probe the 'disorder-to-order transition' of the SARS- CoV-2 FLCS $S_{\text {Spike }}$ loop upon Furin binding}

Finally, the paper takes the opportunity to demonstrate a novel use of the legendary Ramachandran Plot (RP) [51] in probing the 'disorder-to-order transition' of the SARS-CoV-2 FLCS $_{\text {spike }}$ loop upon Furin binding. The unbound disordered state was taken to be the ensemble of 500 'all-atom' SARS-CoV-2 Spike atomic models (see section 3.2) built with its experimentally missing patches modeled with uniquely varied loop-conformations (see section 2.2, Materials and Methods). On the other hand, 500 time-frames sampled at equal temporal interval of 600 ps from the 300 ns MD simulation trajectory of the Spike-Furin complex (simulated from the rank-1 docked pose in the ClusPro blind-docking) were assembled to represent the bound (presumably ordered) state. The Ramachandran backbone torsion angles ( $\Phi$, $\psi$ ) were then computed for each atomic model under each ensemble (bound, unbound) for all

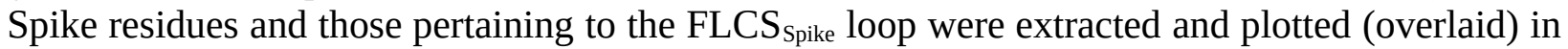
the RP (Figure 7). The overlaid distributions clearly show more scatter for $(\Phi, \psi)$ points in the unbound (Figure 7.A) compared to bound (Figure 7.B) states. In addition, a re-view of the RPs were felt necessarily important with a sense of contiguity for the connected pentapeptide sequence motif (-681 PRRAR $\left._{685}{ }^{-}\right)$embedded in the FLCS $_{\text {Spike }}$ loop. Such a sense of contiguity is also essential in terms of backbone tracing in protein crystallography [118] and depicting secondary structural elements [119]. To that end, a simple line-drawing of the successively connected residues belonging to the $-\mathrm{P}_{681}-\mathrm{R}_{682}-\mathrm{R}_{683}-\mathrm{A}_{684}-\mathrm{R}_{685}-$ pentapeptide motif was performed (Figure S8, Supplementary Materials), over and above the standard 'scattered points representation' of the RP.

For the unbound state, the successive points clearly hovers around extreme ends of the RP resembling a highly multi modal distribution in terms of occupying different regions in RP indicating high structural conflicts or disorder. In comparison, the same successive points clearly gets shrunk into a constrained distribution for the Furin-bound state, directed to an extended 'generously allowed' region [120] of the RP. More interestingly, this extended region almost perfectly maps to the extended bridged territory of the originally proposed allowed regions [51] for beta-sheets and right handed alpha- (as well as $3_{10^{-}}$) helices upon the relaxation of the bondangle, tau ( $\tau$ : $\mathrm{N}-\mathrm{C} \alpha-\mathrm{C}$ ) [121]. Motivated by these very interesting observations, we further

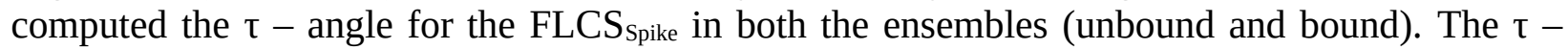
angle in the Furin-bound state (time-averaged) was indeed found to be more relaxed $\left(<\tau_{\text {Furin-bound }}>\right.$ : 109.6 ${ }^{\circ}$; $\mathrm{SD}^{9}: 4.0^{\circ}$ ) and trending to its ideal value of $109.5^{\circ}$ for a tetrahedral $\mathrm{sp}^{3}$ carbon [122], compared to its unbound state where the average value $\left(<_{\text {unbound }}>\right.$ : $107.4^{\circ}$; SD: $\left.2.3^{\circ}\right)$ was somewhat left-shifted from its tetrahedral ideal value. When surveyed for the ${ }_{6}{ }_{61} \mathrm{PRRAR}_{685^{-}}$ pentapeptide motif, the two $<\tau>$ values were even more separated with similar ratio of their standard deviations ( $<\tau_{\text {unbound }}>$ : $107.9^{\circ}$; SD: $2.4^{\circ} ;<_{\text {Furin-bound }}>$ : $110.8^{\circ}$; SD: $4.2^{\circ}$ ). In both cases (FLCS $_{\text {Spike, }}{ }_{681}$ PRRAR $_{685}{ }^{-}$), the standard deviations were 1.7 times more in the bound state (i.e., more relaxed $\tau$ - angles) than in the unbound state. Moreover, from the overlaid RPs plotted for

9 SD: Standard Deviation 


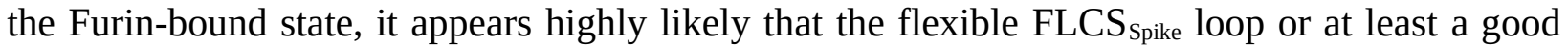
part of the loop is in dynamic equilibrium with multiple short transient secondary structural elements (e.g., short helical turns, beta-strands etc.) in its bound state. Given the trends in $\{\Phi$, $\psi\}$, rationalized by the comparatively relaxed $\tau$ - angle in the bound state, this appears especially relevant to the pentapeptide (-681 $\left.\mathrm{PRRAR}_{685^{-}}\right)$motif (Figure 7.B).

Subsequent to the visual inspection and comparison, the RP derived parameters (see section 2.7, Materials and Methods) were computed for the FLCS ${ }_{\text {Spike }}$ patch independently for each state (unbound and Furin-bound) to quantify the distribution of $(\Phi, \psi)$ points spanning across the two plots (Figure 7.A \& 7.B) and to assess whether this difference is of any significance. From definition (see section 2.7, Materials and Methods), smaller values of $\left|\delta_{c}\right|$ (say, $<30^{\circ}$ ) statistically indicates that the consecutive residues are conformationally alike or close which effectively leads to a local structural coherence and relative structural order for the FLCS Spike, $_{\text {, }}$ while a larger value suggest regular structural conflicts and consequently structural disorder. Thus, from definition, $\left|\delta_{c}\right|$ also gives an estimate of how much the FLCS $S_{\text {Spike }}$ is conformationally varied (or, in other words, distributed among varying structural conformations) on an average. Lesser values of $\left|\delta_{c}\right|$ indicate greater tendencies (on an average) of the FLCS ${ }_{\text {Spike }}$ to attain closely related structural conformations, while as the value increases, structural degeneracy [50] is manifested within the FLCS $_{\text {Spike }}$.

All the RP-derived parameters (see section 2.7, Materials and Methods) are spread descriptors of some sort and all of them unequivocally drop (i.e., shrink) in the bound state (Table 4) compared to the unbound state. The relative decrease from state-1 (Spike, unbound) to state-2 (Spike, Furin-bound) in this two-state transition is $32.5 \%$ in $\left\langle\delta>_{3 q}\right.$ and $55.7 \%$ in $\langle\delta\rangle_{9 \mathrm{~d}}$. This indicates that the distance ( $\delta$, defined in the $\Phi-\psi$ space) by which $90 \%$ of points are seperated in the two RPs (states) is increased more than 1.5 times in the unbound state, compared to the bound state. Together, the visual and the quantitative analyses clearly and directly portray (from actual structural dynamics data) the transition of the unbound disordered FLCS Spike $_{\text {to a relatively }}$ ordered Furin-bound state in SARS-CoV-2 Spike.

Lastly, to render a statistical significance to the change in the obtained distributions of $(\Phi, \psi)$ points in the RP associated with the two state transition (unbound $\rightarrow$ bound) of the FLCS Spike $x^{2}$ test was performed. Ten distinct bins corresponding to disjoint regions in the RP was considered. We adapted the Procheck [123] version of the RP to reproduce the Ramachandran $(\Phi, \psi)$ contours (Figure 7) and used the MATLAB inbuilt function 'inpolygon' for the frequency distribution of $(\Phi, \psi)$ points into these bins. For reasons of simplicity, the later-extended generously allowed regions [120,123] of the RP were avoided. The 10 bins thus represented 3 allowed regions for regular secondary structural elements ( $\beta$-sheets, R $\alpha$-helices, L $\alpha$-helics $)^{10}, 6$ partially allowed regions (of largely varying areas) across the plot, and, the entire left-over disallowed region, pulled into the $10^{\text {th }}$ bin. The null hypothesis was taken to be 'no or little (i.e., insignificant) changes caused in the unbound $(\Phi, \psi)$ points $\left(F L C S_{\text {spike }}\right)$ upon binding to Furin (i.e., Expected: unbound; Observed: bound)'. The $\chi^{2}$ (see section 2.8, Materials and Methods)

10 R: Right handed; L: Left handed 
value obtained from the differential counts (Figure 7) of points (unbound $\rightarrow$ bound) in this 10bin distribution $\left(\mathrm{df}^{11}=9\right)$ was found to be 3650.32 which is 131 times higher than that of the upper-tail critical $\chi^{2}$ value for $\mathrm{df}=9$ at $99.9 \%$ level of significance $\left(\chi_{0.001}^{2}=27.88\right)$. Based on these numbers, the null hypothesis was rejected which should mean that the 'unbound $\rightarrow$ bound' change was indeed significant in the $F L C S_{\text {spike }}$ in terms of their relative RP distributions even at the $99.9 \%$ level. In other words, the 'disorder $\rightarrow$ order transition' of the $F L C S_{\text {Spike }}$ upon binding to Furin was evident and unmistakable.

The RP has previously been used to probe transitions among $\alpha$-helix, $\Pi$-helix and turns in context to the Phosphorylation of Smooth Muscle Myosin [124]. Having said that, the visual impact of simple line-drawing (to portray sequence contiguity) as well as the collective use of RP derived metrices, to the best of our knowledge and belief, together presents yet another novel use of the evergreen and multifaceted Ramachandran Plot.

\section{Conclusion and Perspective}

In parallel to the ongoing efforts to find a sustainable therapeutic solution to curb the coronavirus pandemic, debates are also ongoing regarding the origin of SARS-CoV-2. The current paradigm is that of an accidental 'lab escape' of SARS-CoV-2 giving rise to COVID-19 (from an ongoing 'gain of function' mutational studies) which has found great support from Genome comparison studies of late (see Introduction) revealing the sudden emergence of the ${ }_{681}$ PRRAR $_{685}$ motif in the SARS-CoV-2 Spike, absent in other related respiratory viruses. The

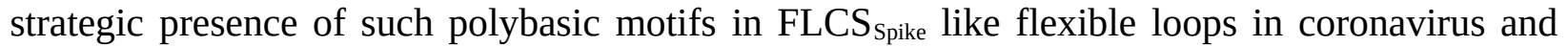
other related respiratory viral lineages leads to local protein disorder [21], intrinsic to these activation loops and the one in SARS-CoV-2 is believed to play a key role in the drastic increase in viral host cell entry and transmissibility. To the very best of our knowledge, the current study is the first of its kind that entraps a 'disorder-to-order transition' in the SARS-CoV-2 FLCS Spike while it undergoes host Furin binding that is optimized for a more efficient proteolytic cleavage of its S1/S2 junction than that in SARS-CoV. The optimization and the consequent increase in proteolytic cleavage efficiency is unambiguous from all analyses performed (sections 3.5-3.8) but is perhaps the most clear and direct from the fairly negative $\Delta \Delta \mathrm{G}_{\text {binding }}$ values returned from the two events (section 3.7). The study further reveals the key role of dynamically interchangeable, persistent salt-bridges at the Spike-Furin interface - which seem to be an evolutionarily conserved feature of the coronavirus lineage and is substantially enhanced in the case of SARS-CoV-2 due to the presence of the three arginines (R682, R683, R685) in the ${ }_{681}$ PRRAR $_{685}$ motif amid its FLCS ${ }_{\text {Spike }}$. The host Furin, orchestrated with a preponderance of exposed amenable anionic residues (E230, E236, D259, D264, D306) strategically positioned around its catalytic triad overwhelmingly favors polybasic disordered substrates like that of the ${ }_{681}$ PRRAR $_{685}$ motif (SARS-CoV-2) for binding, cleavage and consequent host cell entry of the virus (sections 3.5-3.6). The resultant Spike-Furin interfacial salt-bridges not only serves as a prominent enthalpy source for the process (compensating for the entropic loss of the FLCS Spike undergoing 'disorder-to-order transition') but also favors the system to retain its characteristic metastabilities favorable for proteolytic cleavages targeted at flexible protein loops (section 3.7). The current study also helps to open up new research avenues across other related protease

11 df: Degree of Freedom 
families harboring such cleavage loops, as to whether this revealed metastabilities also holds true in them. The findings are perfectly consistent with the established theories of salt-bridge dynamics in context to IDPs serving to retain their characteristic structural plasticity by the continuous triggering of phase transitions among their self-similar disordered states [49,50]. Further, from the combined results of salt-bridge and thermodynamic analyses (see section 3.6, 3.7) it strongly appears that the Furin cleavage seeks opportunities for transient formation of

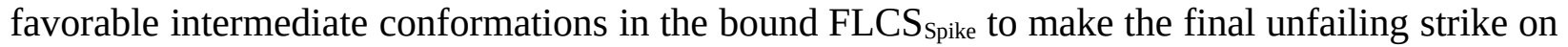
the desired peptide bond. The probabilities to have this successful strike is naturally far greater in SARS-CoV-2 (than in SARS-CoV) since it has the more intense salt-bridge networks formed and sustained in its Spike-Furin interface (for the presence of the ${ }_{681} \mathrm{PRRAR}_{685}$ - arginines). These findings further rationalize the substantially greater extent of cleavage (59.6\%) of the SARS-CoV-2 Spike (into its S1/S2 products) in the wild-type virion than in its $\triangle$ PRRA mutant (14.5\%) [33]. In conclusion, over and above offering a novel perspective into the coronavirus molecular evolution, the study also makes the SARS-CoV-2 Spike-Furin interaction mechanistically insightful adding new dimensions to the existing theories of proteolytic cleavages per se. 


\section{Display items (Figures and Tables)}

\section{Figures}

Figure 1. PrDos disorder probability scores plotted for coronavirus Spike sequences. The sequences cover the entire non-redundant sequence space up to the evolution of SARS-CoV-2 for the pentapeptide sequence motifs (e.g., ${ }_{681} P R R A R_{685}$ in SARS-CoV-2) embedded in FLCS Spike. Panel (A) portrays the residue-wise disorder probabilities (with the FLCS $_{\text {Spike }}$-residues highlighted with deferentially colored thick circles) while panel (B) plots their mean values

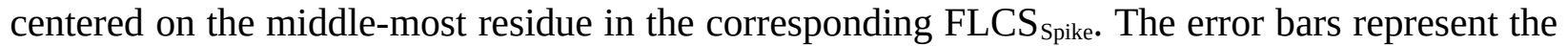
corresponding standard deviations.
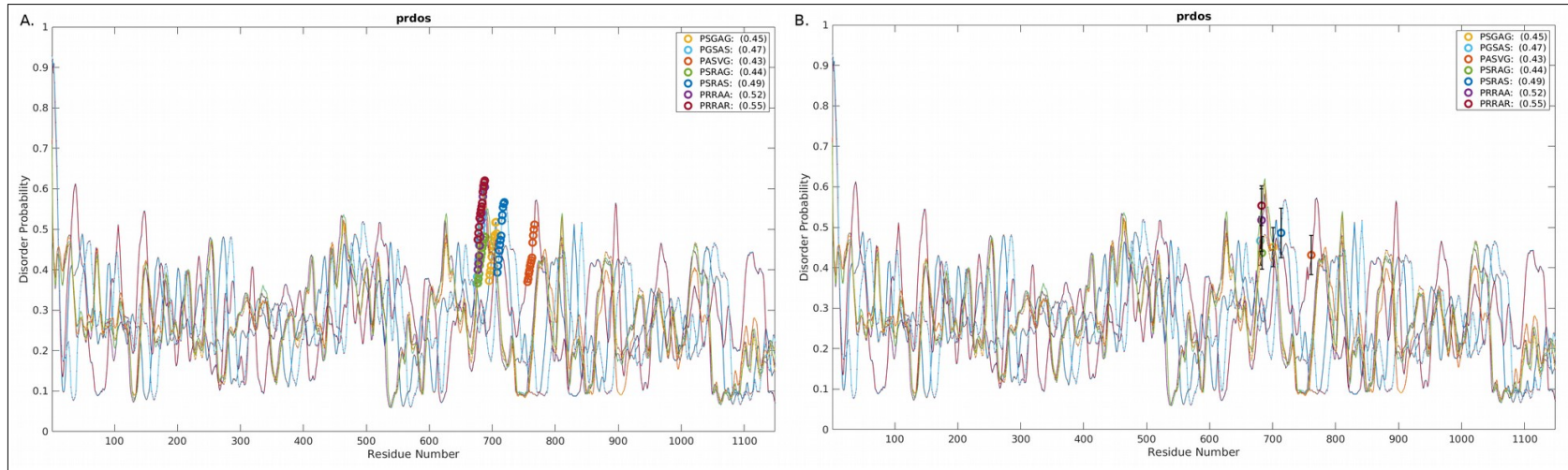
Figure 2. The proposed dockable surface groove for FLCS $_{\text {Spike }}$ in Furin, proximal to its catalytic triad. The catalytic triad (D153, H194, S368) is highlighted dots and labeled in PyMol color: 'density' (panel A) over and above the cartoon display. All exposed and partially exposed anionic residues are shown in 'balls and sticks'. Among these, the ones that are in close vicinity to the dockable groove surface (panel $\mathbf{B}$ ) are further labeled with residue identities in both panels. These residues are amenable to form interfacial salt-bridges with the FLCS spike $_{\text {-arginines }}$ (in ${ }_{681}$ PRRAR $_{685}$ ) and collectively add to the potential of forming dynamically interchangeable ionic bond networks at the Spike-Furin interface.
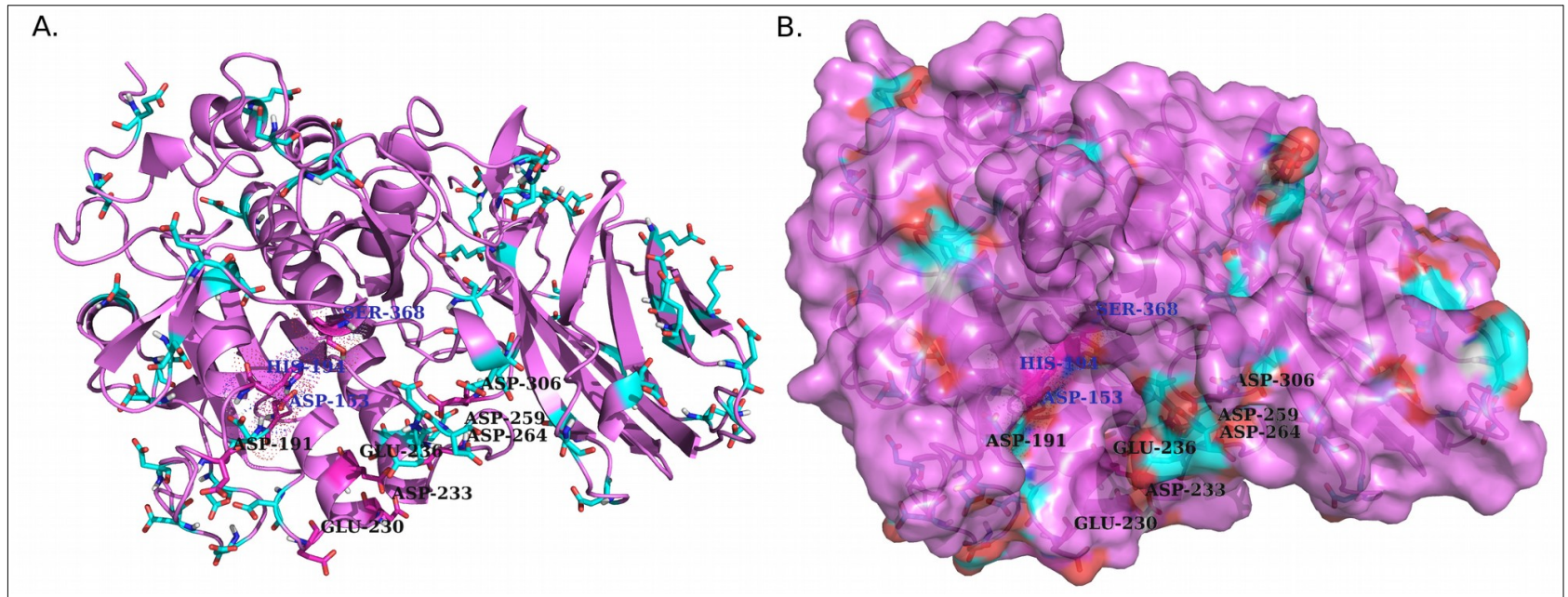


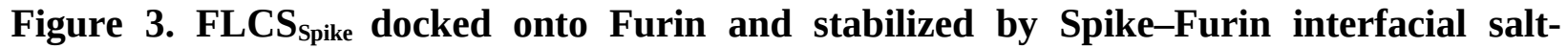

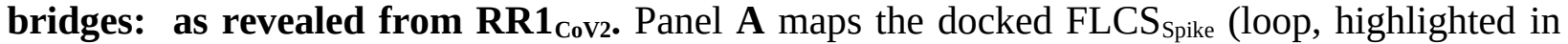
yellow, flanked at either end by beta-strands colored in cyan) at the Furin docking site in RR1 $1_{\mathrm{Cov} 2}$ while the rest of the Spike that is visible in this closeup view is in ribbon (PyMol). A direct visual comparison can be made between Figure 2 portraying the proposed dockable surface groove (see corresponding main-text, section 3.4) near the Furin catalytic triad and Figure 3.A

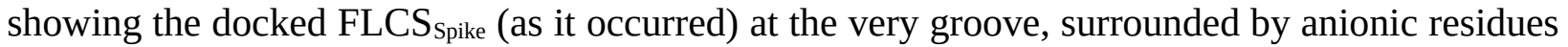
amenable to form salt-bridges with the FLCS $_{\text {spike }}$ - arginines (R682, R683, R685). Panel B highlights the highest persistence Spike-Furin interfacial salt-bridges (thick yellow dashed lines) for the three arginines in the ${ }_{681} \mathrm{PRRAR}_{685}\left(\mathrm{FLCS}_{\text {Spike }}\right)$ along its $300 \mathrm{~ns}$ MD simulation trajectory (produced from $\mathrm{RR} 1_{\mathrm{Cov} 2}$ ). Only a close up view of the interface is portrayed to highlight the key (highly persistent) salt-bridges (pers $>0.85$ ) sustaining the interface. Only the Furin - proximal part (FLCS $_{\text {spike }}$ with short flanking regions at either end) of the Spike (chain S) is shown in magenta cartoon (with R682, R683, R685 highlighted in 'balls and sticks') while the Furin chain (chain F) is drawn in green cartoon with its counter-ionic residues involved in the (aforementioned) key Spike-Furin interfacial salt-bridges highlighted in 'balls and sticks'. Residues comprising the catalytic triad are presented in 'balls and sticks' colored in navy blue and highlighted by dot surface points. Figures were built in PyMol.

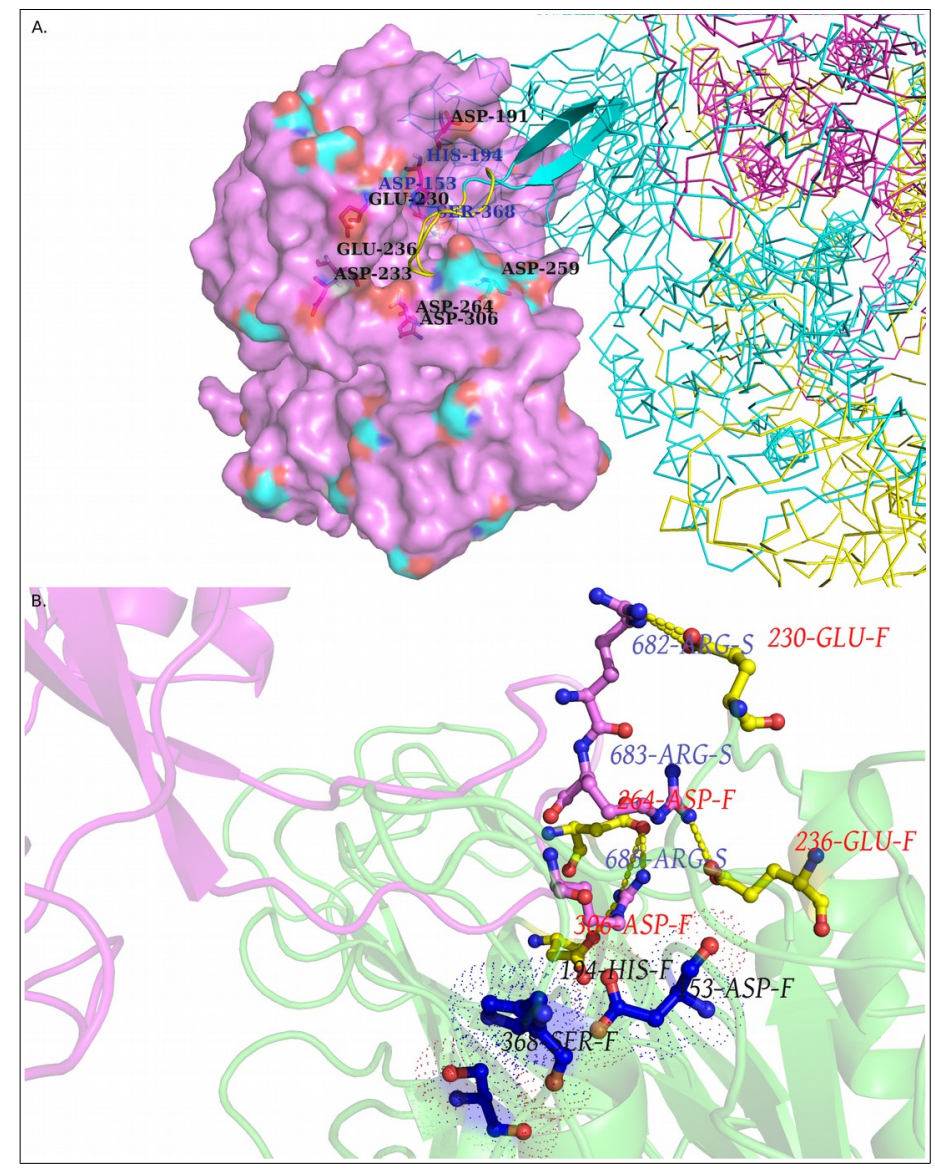


Figure 4. The anionic and the catalytic triad of the Spike - bound Furin in correlated movements throughout the 300 ns MD simulation trajectory (RR1).

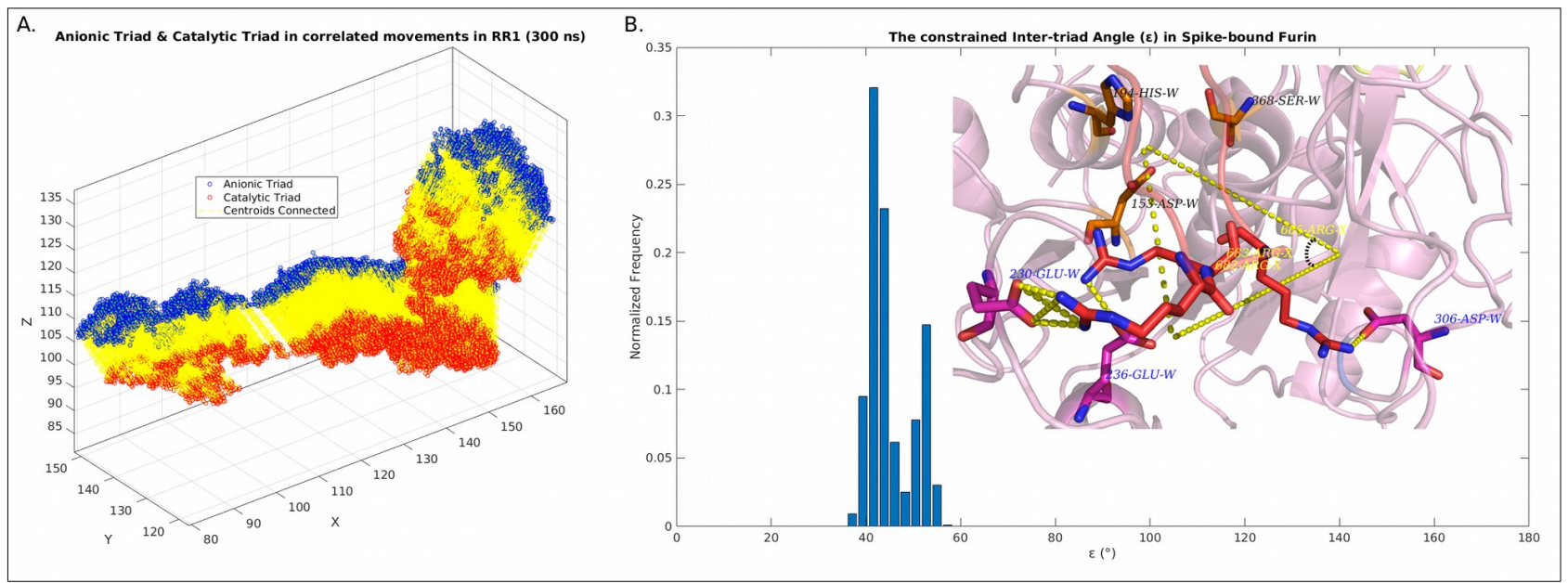


Figure 5. Densely connected composite ionic bond motifs in the persistent salt-bridges formed at the Spike-Furin interface. Panels A. and B. plots a representative snapshots of the interface (with a close-up view for the ionic bond motifs) randomly picked from the trajectories of RR1 $1_{\mathrm{Cov} 2}$ and ZR1 $1_{\mathrm{Cov} 2}$ respectively.

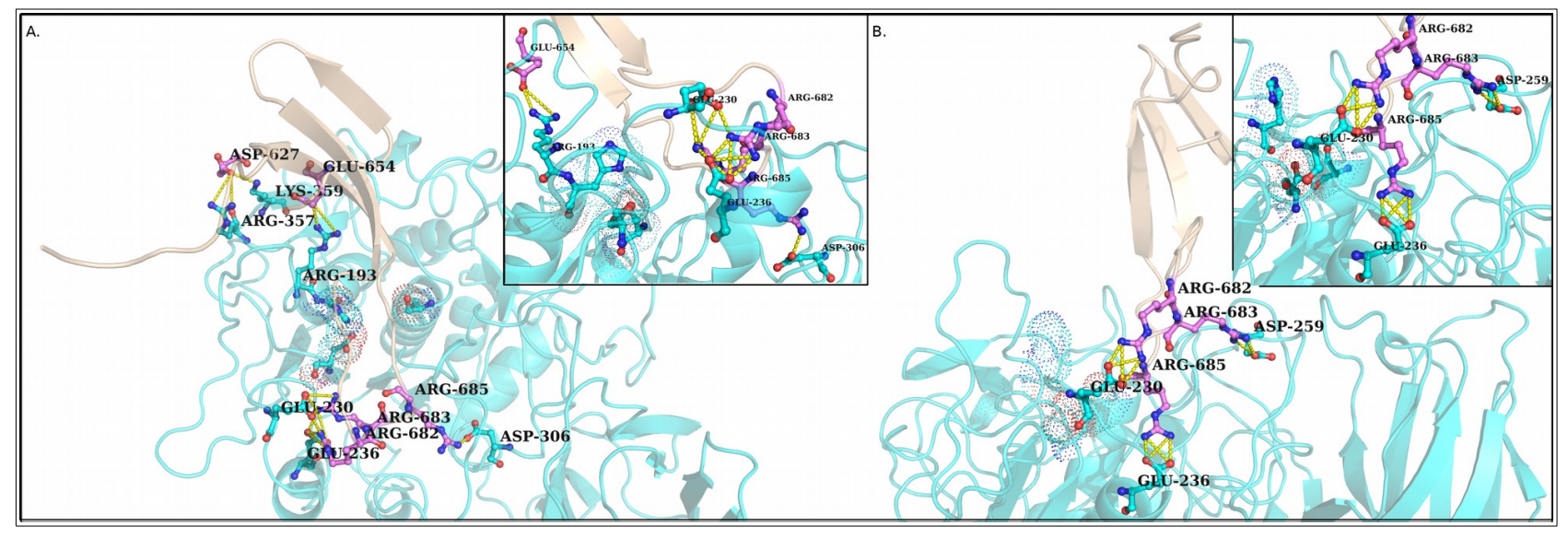


Figure 6. Interaction energy profiles (FoldX) for the top re-ranked Spike-Furin complexes

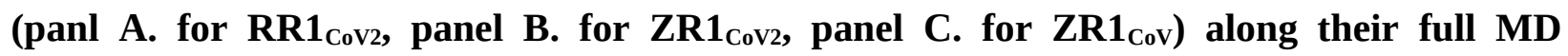
simulation trajectories (300 ns, $100 \mathrm{~ns}, 300 \mathrm{~ns})$. The different transition enthalpic $\left(\Delta \mathrm{H}_{\mathrm{vdw}}\right.$, $\Delta \mathrm{H}_{\text {elec}}$ ) and entropic $\left(\mathrm{T} \Delta \mathrm{S}_{\mathrm{mc}}, \mathrm{T} \Delta \mathrm{S}_{\mathrm{sc}}\right)$ terms along with the net $\Delta \mathrm{G}_{\text {binding }}$ are plotted in different colors as has been mentioned in the corresponding legend-boxes. All thermodynamic parameters are essentially energy terms and are plotted in the units of $\mathrm{kcal} \mathrm{mol}^{-1}$.

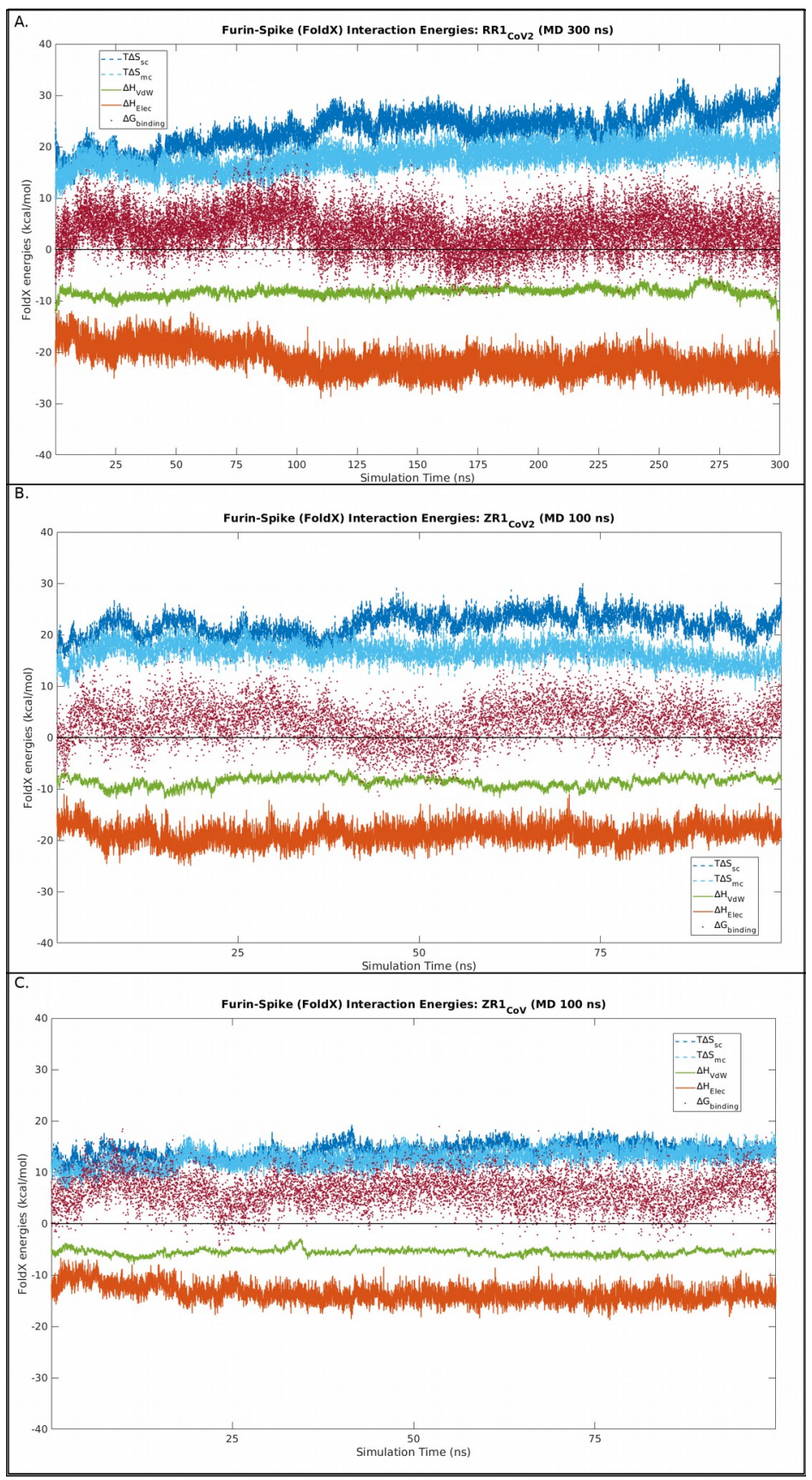


Figure 7. Overlaid Ramachrandran Plots for FLCS $_{\text {Spike }}$ pertaining to (A) unbound and (B) Furin-bound Spike states. Each plot is overlaid with 100 atomic models belonging to the same state (unbound or bound). Within each individual plot (i.e., pertaining to each atomic model), the $\{\Phi, \Psi\}$ points for residues in the FLCS $_{\text {Spike }}$ loop is plotted as black dots encircled by green while those for residues belonging to the $-\mathrm{P}_{681}-\mathrm{R}_{682}-\mathrm{R}_{683}-\mathrm{A}_{684}-\mathrm{R}_{685}-$ pentapeptide motif are highlighted by red dots encircled by blue.
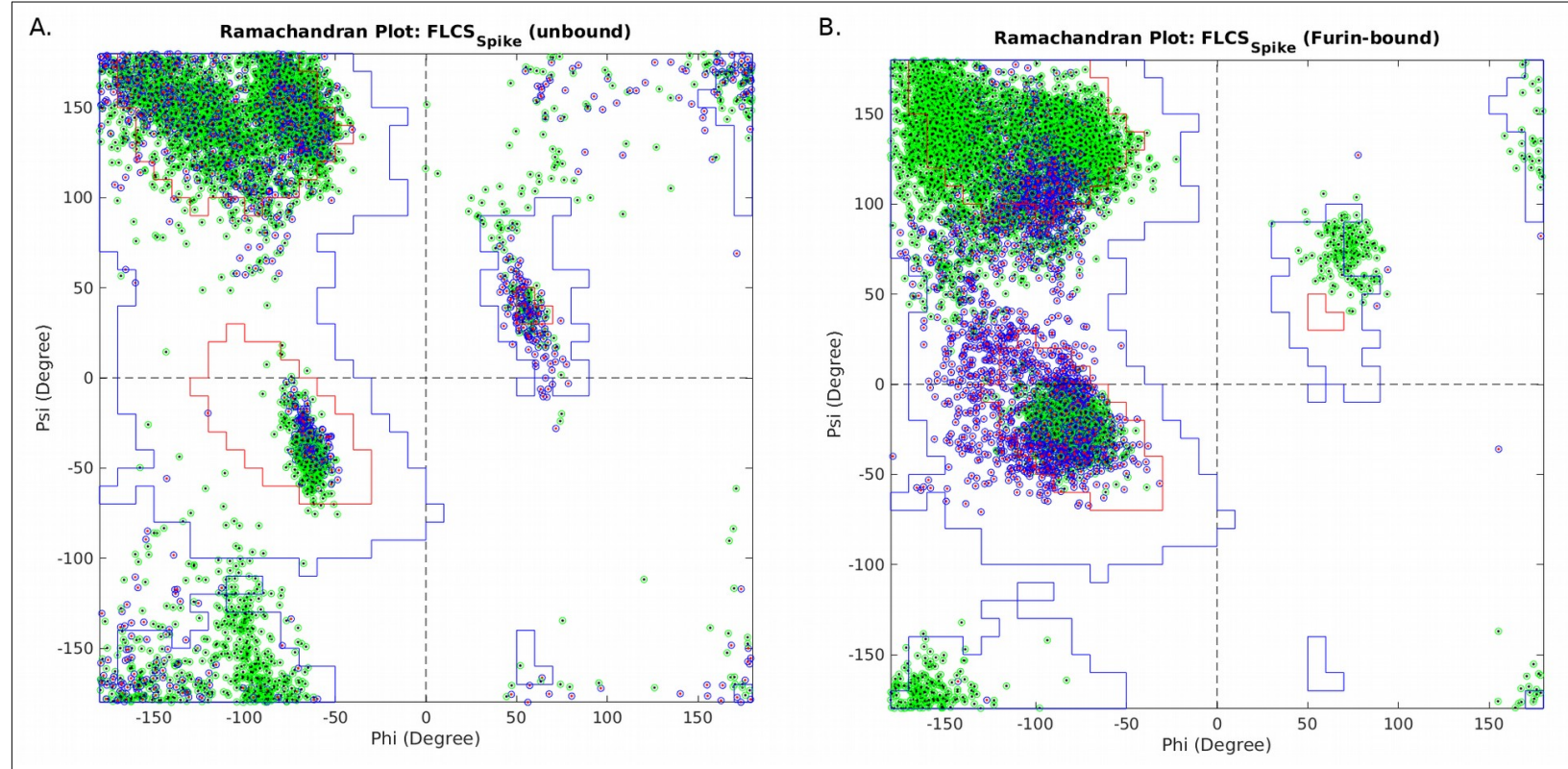
Figure 8. Distribution of differential counts obtained from binning of the RP for unbound and bound states of $\mathbf{F L C S}_{\text {spike. }}$ For binning details please refer to main-text. Bin-1, 2 \& 8 represents Ramachandran allowed regions for $\beta$-sheets, R $\alpha$-helices, $L \alpha$-helices while 3-7, 9 maps to 6 disjoint partially allowed regions and bin-10 stands for the pulled-down disallowed region.

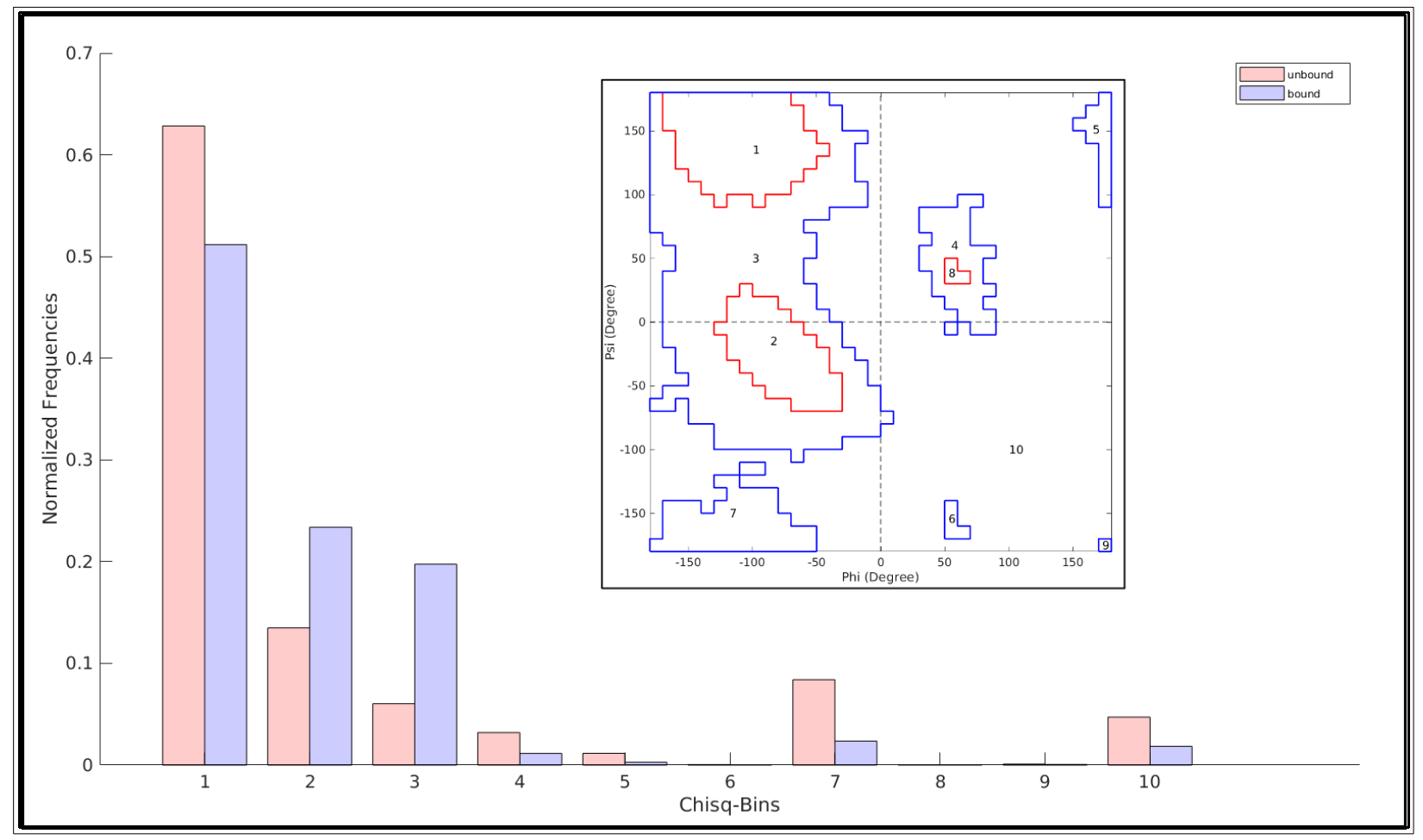




\section{Tables}

Table 1. Persistence and average contact intensities of all unique salt-bridges at the SARSCoV2 Spike-Furin interface for $R R 1_{\mathrm{CoV} 2}$ (the top re-ranked docked pose) along its $300 \mathrm{~ns}$ MD simulation trajectory. ' $-S$ ' \& ' $-F$ ' in the salt-bridge descriptor strings refer to the receptor and the ligand chains respectively. Rows corresponding to the Arginine-salt-bridges falling within the pentapeptide ${ }_{681} \mathrm{PRRAR}_{685}$ motif (activation loop of FLCS ${ }_{\text {Spike) }}$ ) is highlighted in three different font colors for R682, R683, R685.

\begin{tabular}{|c|c|c|c|c|}
\hline Salt-bridge & TotC & Frames $_{\boldsymbol{p}}$ & ACI & Pers \\
\hline $294-A S P-S \leftrightarrow 349-L Y S-F$ & 1 & 1 & 1.00 & 0.00003 \\
\hline $683-A R G-S \leftrightarrow 191-A S P-F$ & 4 & 4 & 1.00 & 0.00013 \\
\hline $654-G L U-S \leftrightarrow 357-A R G-F$ & 23 & 23 & 1.00 & 0.00077 \\
\hline $683-A R G-S \leftrightarrow 264-A S P-F$ & 196 & 64 & 3.06 & 0.00213 \\
\hline $682-A R G-S \leftrightarrow 233-A S P-F$ & 73 & 73 & 1.00 & 0.00243 \\
\hline $654-G L U-S \leftrightarrow 359-L Y S-F$ & 149 & 131 & 1.14 & 0.00437 \\
\hline $683-A R G-S \leftrightarrow 230-G L U-F$ & 247 & 185 & 1.34 & 0.00617 \\
\hline $685-A R G-S \leftrightarrow 236-G L U-F$ & 251 & 148 & 1.70 & 0.00493 \\
\hline $214-A R G-S \leftrightarrow 112-G L U-F$ & 729 & 276 & 2.64 & 0.00920 \\
\hline $627-A S P-S \leftrightarrow 359-L Y S-F$ & 1247 & 1173 & 1.06 & 0.03910 \\
\hline $627-A S P-S \leftrightarrow 357-A R G-F$ & 2763 & 1460 & 1.89 & 0.04867 \\
\hline $682-A R G-S \leftrightarrow 236-G L U-F$ & 6374 & 1657 & 3.85 & 0.05523 \\
\hline $685-A R G-S \leftrightarrow 264-A S P-F$ & 16141 & 7146 & 2.26 & 0.23820 \\
\hline $654-G L U-S \leftrightarrow 193-A R G-F$ & 45873 & 19387 & 2.37 & 0.64623 \\
\hline $685-A R G-S \leftrightarrow 306-A S P-F$ & 37148 & 26969 & 1.38 & 0.89897 \\
\hline $683-A R G-S \leftrightarrow 236-G L U-F$ & 43275 & 25546 & 1.69 & 0.85153 \\
\hline $682-A R G-S \leftrightarrow 230-G L U-F$ & 75112 & 27762 & 2.71 & 0.92540 \\
\hline
\end{tabular}

TotC: Total Counts (Ion-pairs)

Frames $_{p}$ : Number of frames the salt-bridge (Residue-Pair) is found in

ACI: Average Contact Intensity $=$ TotC/ Frames

Pers: Persistence $=$ Frames $_{p} /$ Frames $_{t}$

Frames $_{t}=$ Total number of frames $=30000$ (sampled at 10 ps interval) 
Table 2. Structure-based equilibrium thermodynamic parameters (FoldX) obtained from the $\mathrm{MD}$ simulation trajectories of $\mathrm{RR} 1_{\mathrm{CoV} 2}, \mathrm{ZR}_{\mathrm{CoV} 2}, \mathrm{ZR} \mathbf{1}_{\mathrm{Cov}}$ representing the Spike-Furin binding in SARS-CoV2 (subject, cross-validation) and SARS-CoV (baseline) respectively. Time-series averages (in kcal $\mathrm{mol}^{-1}$ ) over the corresponding trajectories for each subject $\left(\mathrm{RR} 1_{\mathrm{CoV} 2}, \mathrm{ZR} 1_{\mathrm{CoV} 2}, \mathrm{ZR} 1_{\mathrm{Cov}}\right)$ are given for each energy term (including enthalpic, entropic, freeenergy terms) with their standard deviations given in parenthesis.

\begin{tabular}{|c|c|c|c|c|c|}
\hline & $<\Delta \mathrm{H}_{\mathrm{vdw}}>$ & $<\Delta \mathrm{H}_{\mathrm{elec}}>$ & $<\Delta \mathrm{S}_{\mathrm{mc}}>$ & $<\Delta \mathrm{S}_{\mathrm{sc}}>$ & $<\Delta \mathrm{G}_{\text {binding }}>$ \\
\hline $\mathrm{RR} 1_{\mathrm{CoV} 2}$ & -21.722 & -8.296 & 17.890 & 23.386 & 3.687 \\
& $(2.669)$ & $(0.871)$ & $(2.276)$ & $(3.432)$ & $(3.868)$ \\
\hline $\mathrm{ZR} 1_{\mathrm{CoV} 2}$ & -18.748 & -8.617 & 16.498 & 22.004 & 3.043 \\
& $(1.871)$ & $(0.956)$ & $(1.889)$ & $(2.243)$ & $(3.843)$ \\
\hline $\mathrm{ZR}_{\mathrm{CoV}}$ & -13.566 & -5.556 & 12.663 & 14.218 & 6.468 \\
& $(1.695)$ & $(0.582)$ & $(1.746)$ & $(1.506)$ & $(3.135)$ \\
\hline
\end{tabular}


Table 3. Average entropic changes pertaining to the Spike-Furin interaction in RR1 $1_{\mathrm{CoV} 2}$ and $\mathbf{Z R} 1_{\mathrm{Cov} 2}$. The statistics is done over the entire $300 \mathrm{~ns}$ trajectory (RR1). Standard deviations given in parentheses. The subscripts in the $\Delta \mathrm{S}$ terms refer to mc: main-chain, sc: side-chain.

\begin{tabular}{|c|c|c|c|c|c|c|c|c|c|}
\hline & \multicolumn{3}{|c|}{$\begin{array}{c}\text { Receptor } \\
\text { (Spike) }\end{array}$} & \multicolumn{3}{c|}{$\begin{array}{c}\text { Ligand } \\
\text { (Furin) }\end{array}$} & \multicolumn{3}{c|}{$\begin{array}{c}\text { Complex } \\
\text { (Spike-Furin) }\end{array}$} \\
\cline { 2 - 10 } & $\mathrm{RR}_{\mathrm{CoV} 2}$ & $\mathrm{ZR}_{\mathrm{CoV} 2}$ & $\mathrm{ZR}_{\mathrm{CoV}}$ & $\mathrm{RR}_{\mathrm{CoV} 2}$ & $\mathrm{ZR}_{\mathrm{CoV} 2}$ & $\mathrm{ZR}_{\mathrm{CoV}}$ & $\mathrm{RR}_{\mathrm{CoV} 2}$ & ZR1 $_{\mathrm{CoV} 2}$ & $\mathrm{ZR}_{\mathrm{CoV}}$ \\
\hline $\begin{array}{c}<\Delta \mathrm{S}_{\mathrm{mc}}> \\
(\mathrm{kcal}\end{array}$ & 6573.9 & 6564.5 & 6344.2 & 2691.4 & 2701.4 & 2613.6 & 17.9 & 16.5 & 12.7 \\
$\left.\mathrm{~mol}^{-1}\right)$ & $(21.8)$ & $(23.8)$ & $(23.5)$ & $(16.1)$ & $(14.8)$ & $(14.3)$ & $(2.3)$ & $(1.9)$ & $(1.8)$ \\
\hline $\begin{array}{c}<\mathrm{S}_{\mathrm{sc}}> \\
(\mathrm{kcal}>\end{array}$ & 2207.2 & 2185.1 & 2077.4 & 860.8 & 846.8 & 821.9 & 23.4 & 22.0 & 14.2 \\
$\left.\mathrm{~mol}^{-1}\right)$ & $(12.9)$ & $(14.7)$ & $(21.4)$ & $(6.5)$ & $(7.4)$ & $(9.1)$ & $(3.4)$ & $(2.2)$ & $(1.5)$ \\
\hline
\end{tabular}




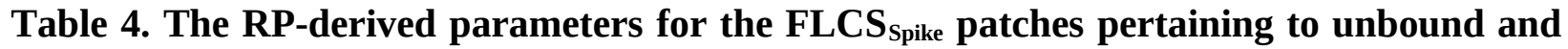
bound Spike states in SARS-CoV-2. The parameters in the header-cells $\left(\left|\delta_{c}\right|, \sigma\left(\delta_{c}\right),<\delta>_{\text {median, }}\right.$, $<\delta>_{3 q},<\delta>_{9 d}$ ) are as defined in section 2.7, Materials and Methods.

\begin{tabular}{|c|c|c|c|c|}
\hline $\begin{array}{c}\text { Spike } \\
\text { States }\end{array}$ & $\begin{array}{c}\left|\delta_{c}\right| \\
\left(\sigma\left(\delta_{\mathrm{c}}\right)\right)\end{array}$ & $<\delta>_{\text {median }}$ & $<\delta>_{3 \mathrm{q}}$ & $<\delta>_{9 \mathrm{~d}}$ \\
\hline $\begin{array}{c}\text { State-1 } \\
\text { (Unbound) }\end{array}$ & $\begin{array}{c}138.5 \\
(30.6)\end{array}$ & 101.0 & 212.0 & 305.2 \\
\hline $\begin{array}{c}\text { State-2 } \\
\text { (Bound) }\end{array}$ & $\begin{array}{c}118.7 \\
(26.2)\end{array}$ & 94.7 & 160.5 & 196.0 \\
\hline $\begin{array}{c}\text { Relative } \\
\text { increase } \\
\text { from state-1 } \\
\text { to state-2 } \\
(\%)\end{array}$ & -16.7 & -6.7 & -32.1 & -55.7 \\
\hline
\end{tabular}




\section{Acknowledgment and Funding}

We convey our sincerest gratitude to Prof. Raghavan Varadarajan, Molecular Biophysics Unit, IISc, Bangalore, India and Prof. Dhananjay Bhattacharyya, Computational Science Division, Saha Institute of Nuclear Physics, Kolkata, India (retired) for their constant motivations and helpful discussions during the work. The project was self-funded.

\section{Author's Contributions}

Conceptualization: SB; Design: SB, AB; Setting up and performing the MD simulations: SR; Formal analysis and investigation: SB, AB, SR, PG; Methodology and validation: SB, AB, SR; Literature review: PG, SR, SB; Writing-original draft preparation: SB, AB; writing-review and editing SB, AB, PG, SR. All authors have read and approved the final manuscript.

\section{Supplementary Information}

Enclosed, please find the Supplementary Materials File (in PDF format) containing eight Supplementary Figures, six Supplementary Tables and one Supplementary Video.

\section{References}

1. Balaram, P. The Murky Origins of the Coronavirus SARS-CoV-2, the Causative Agent of the COVID-19 Pandemic. Curr. Sci. 2021, 120, 4.

2. Zhou, P.; Yang, X.-L.; Wang, X.-G.; Hu, B.; Zhang, L.; Zhang, W.; Si, H.-R.; Zhu, Y.; Li, B.; Huang, C.-L.; et al. A Pneumonia Outbreak Associated with a New Coronavirus of Probable Bat Origin. Nature 2020, 579, 270-273, doi:10.1038/s41586-020-2012-7.

3. Xu, X.; Chen, P.; Wang, J.; Feng, J.; Zhou, H.; Li, X.; Zhong, W.; Hao, P. Evolution of the Novel Coronavirus from the Ongoing Wuhan Outbreak and Modeling of Its Spike Protein for Risk of Human Transmission. Sci. China Life Sci. 2020, 63, 457-460, doi:10.1007/s11427-020-1637-5.

4. Andersen, K.G.; Rambaut, A.; Lipkin, W.I.; Holmes, E.C.; Garry, R.F. The Proximal Origin of SARS-CoV-2. Nat. Med. 2020, 26, 450-452, doi:10.1038/s41591-020-0820-9.

5. Calisher, C.; Carroll, D.; Colwell, R.; Corley, R.B.; Daszak, P.; Drosten, C.; Enjuanes, L.; Farrar, J.; Field, H.; Golding, J.; et al. Statement in Support of the Scientists, Public Health Professionals, and Medical Professionals of China Combatting COVID-19. The Lancet 2020, 395, e42-e43, doi:10.1016/S0140-6736(20)30418-9.

6. The Origin of COVID: Did People or Nature Open Pandora's Box at Wuhan? Bull. At. Sci. 2021.

7. Malaiyan, J.; Arumugam, S.; Mohan, K.; Gomathi Radhakrishnan, G. An Update on the Origin of SARS-CoV-2: Despite Closest Identity, Bat (RaTG13) and Pangolin Derived Coronaviruses Varied in the Critical Binding Site and O-Linked Glycan Residues. J. Med. Virol. 2021, 93, 499-505, doi:10.1002/jmv.26261. 
8. Casadevall, A.; Weiss, S.R.; Imperiale, M.J. Can Science Help Resolve the Controversy on the Origins of the SARS-CoV-2 Pandemic? mBio 2021, 12, doi:10.1128/mBio.01948-21.

9. Imperiale, M.J.; Casadevall, A. Rethinking Gain-of-Function Experiments in the Context of the COVID-19 Pandemic. mBio 2020, 11, e01868-20, doi:10.1128/mBio.01868-20.

10. Bloom, J.D.; Chan, Y.A.; Baric, R.S.; Bjorkman, P.J.; Cobey, S.; Deverman, B.E.; Fisman, D.N.; Gupta, R.; Iwasaki, A.; Lipsitch, M.; et al. Investigate the Origins of COVID-19. Science 2021.

11. Gillum, D.; Moritz, R. Why Gain-of-Function Research Matters Available online: http://theconversation.com/why-gain-of-function-research-matters-162493 (accessed on 10 September 2021).

12. Sciences, B. on L.; Studies, D. on E. and L.; Committee on Science, T.; Affairs, P. and G.; Policy, B. on H.S.; Council, N.R.; Medicine, I. of Gain-of-Function Research: Background and Alternatives; National Academies Press (US), 2015;

13. Engineering a Virus: What Is Gain of Function Research? | Faculty of Sciences | University of Adelaide Available online:

https://sciences.adelaide.edu.au/news/list/2021/07/21/engineering-a-virus-what-is-gain-offunction-research (accessed on 10 September 2021).

14. Willingham, E. Why Scientists Tweak Lab Viruses to Make Them More Contagious Available online: https://www.scientificamerican.com/article/why-scientists-tweak-labviruses-to-make-them-more-contagious1/ (accessed on 10 September 2021).

15. Basu, S.; Chakravarty, D.; Bhattacharyya, D.; Saha, P.; Patra, H.K. Plausible Blockers of Spike RBD in SARS-CoV2-Molecular Design and Underlying Interaction Dynamics from High-Level Structural Descriptors. J. Mol. Model. 2021, 27, 191, doi:10.1007/s00894-02104779-0.

16. Chowdhury, R.; Boorla, V.S.; Maranas, C.D. Computational Biophysical Characterization of the SARS-CoV-2 Spike Protein Binding with the ACE2 Receptor and Implications for Infectivity. Comput. Struct. Biotechnol. J. 2020, 18, 2573-2582, doi:10.1016/j.csbj.2020.09.019.

17. Shang, J.; Ye, G.; Shi, K.; Wan, Y.; Luo, C.; Aihara, H.; Geng, Q.; Auerbach, A.; Li, F. Structural Basis of Receptor Recognition by SARS-CoV-2. Nature 2020, 581, 221-224, doi:10.1038/s41586-020-2179-y.

18. Ou, X.; Liu, Y.; Lei, X.; Li, P.; Mi, D.; Ren, L.; Guo, L.; Guo, R.; Chen, T.; Hu, J.; et al. Characterization of Spike Glycoprotein of SARS-CoV-2 on Virus Entry and Its Immune Cross-Reactivity with SARS-CoV. Nat. Commun. 2020, 11, 1620, doi:10.1038/s41467020-15562-9.

19. Hoffmann, M.; Hofmann-Winkler, H.; Pöhlmann, S. Priming Time: How Cellular Proteases Arm Coronavirus Spike Proteins. Act. Viruses Host Proteases 2018, 71-98, doi:10.1007/978-3-319-75474-1_4.

20. Millet, J.K.; Whittaker, G.R. Physiological and Molecular Triggers for SARS-CoV Membrane Fusion and Entry into Host Cells. Virology 2018, 517, 3-8, doi:10.1016/j.virol.2017.12.015.

21. Cai, Y.; Zhang, J.; Xiao, T.; Peng, H.; Sterling, S.M.; Walsh, R.M.; Rawson, S.; RitsVolloch, S.; Chen, B. Distinct Conformational States of SARS-CoV-2 Spike Protein. Science 2020, doi:10.1126/science.abd4251. 
22. Yuan, Y.; Cao, D.; Zhang, Y.; Ma, J.; Qi, J.; Wang, Q.; Lu, G.; Wu, Y.; Yan, J.; Shi, Y.; et al. Cryo-EM Structures of MERS-CoV and SARS-CoV Spike Glycoproteins Reveal the Dynamic Receptor Binding Domains. Nat. Commun. 2017, 8, 15092, doi:10.1038/ncomms15092.

23. Gui, M.; Song, W.; Zhou, H.; Xu, J.; Chen, S.; Xiang, Y.; Wang, X. Cryo-Electron Microscopy Structures of the SARS-CoV Spike Glycoprotein Reveal a Prerequisite Conformational State for Receptor Binding. Cell Res. 2017, 27, 119-129, doi:10.1038/cr.2016.152.

24. Walls, A.C.; Park, Y.-J.; Tortorici, M.A.; Wall, A.; McGuire, A.T.; Veesler, D. Structure, Function, and Antigenicity of the SARS-CoV-2 Spike Glycoprotein. Cell 2020, 181, 281292.e6, doi:10.1016/j.cell.2020.02.058.

25. Wrapp, D.; Wang, N.; Corbett, K.S.; Goldsmith, J.A.; Hsieh, C.-L.; Abiona, O.; Graham, B.S.; McLellan, J.S. Cryo-EM Structure of the 2019-NCoV Spike in the Prefusion Conformation. Science 2020, 367, 1260-1263, doi:10.1126/science.abb2507.

26. Basu, S.; Saha, P.; Bhattacharyya, D.; Patra, H.K. Plausible Blockers of Spike RBD in SARS-CoV2 - Molecular Design and Underlying Interaction Dynamics from High-Level Structural Descriptors. 2020, doi:10.20944/preprints202009.0006.v1.

27. Papa, G.; Mallery, D.L.; Albecka, A.; Welch, L.G.; Cattin-Ortolá, J.; Luptak, J.; Paul, D.; McMahon, H.T.; Goodfellow, I.G.; Carter, A.; et al. Furin Cleavage of SARS-CoV-2 Spike Promotes but Is Not Essential for Infection and Cell-Cell Fusion. PLOS Pathog. 2021, 17, e1009246, doi:10.1371/journal.ppat.1009246.

28. Peacock, T.P.; Sheppard, C.M.; Brown, J.C.; Goonawardane, N.; Zhou, J.; Whiteley, M.; Consortium, P.V.; Silva, T.I. de; Barclay, W.S. The SARS-CoV-2 Variants Associated with Infections in India, B.1.617, Show Enhanced Spike Cleavage by Furin; 2021; p. 2021.05.28.446163;

29. Nagy, A.; Basiouni, S.; Parvin, R.; Hafez, H.M.; Shehata, A.A. Evolutionary Insights into the Furin Cleavage Sites of SARS-CoV-2 Variants from Humans and Animals. Arch. Virol. 2021, 166, 2541-2549, doi:10.1007/s00705-021-05166-z.

30. How Ominous Is the Omicron Variant (B.1.1.529)? Available online: https://asm.org/Articles/2021/December/How-Ominous-is-the-Omicron-Variant-B-1-1-529 (accessed on 27 December 2021).

31. Bertram, S.; Glowacka, I.; Müller, M.A.; Lavender, H.; Gnirss, K.; Nehlmeier, I.; Niemeyer, D.; He, Y.; Simmons, G.; Drosten, C.; et al. Cleavage and Activation of the Severe Acute Respiratory Syndrome Coronavirus Spike Protein by Human Airway Trypsinlike Protease. J. Virol. 2011, 85, 13363-13372, doi:10.1128/JVI.05300-11.

32. Henrich, S.; Cameron, A.; Bourenkov, G.P.; Kiefersauer, R.; Huber, R.; Lindberg, I.; Bode, W.; Than, M.E. The Crystal Structure of the Proprotein Processing Proteinase Furin Explains Its Stringent Specificity. Nat. Struct. Biol. 2003, 10, 520-526, doi:10.1038/nsb941.

33. Johnson, B.A.; Xie, X.; Bailey, A.L.; Kalveram, B.; Lokugamage, K.G.; Muruato, A.; Zou, J.; Zhang, X.; Juelich, T.; Smith, J.K.; et al. Loss of Furin Cleavage Site Attenuates SARSCoV-2 Pathogenesis. Nature 2021, 591, 293-299, doi:10.1038/s41586-021-03237-4. 
34. Shang, J.; Wan, Y.; Luo, C.; Ye, G.; Geng, Q.; Auerbach, A.; Li, F. Cell Entry Mechanisms of SARS-CoV-2. Proc. Natl. Acad. Sci. 2020, 117, 11727-11734, doi:10.1073/pnas.2003138117.

35. Mustafa, Z.; Zhanapiya, A.; Kalbacher, H.; Burster, T. Neutrophil Elastase and Proteinase 3 Cleavage Sites Are Adjacent to the Polybasic Sequence within the Proteolytic Sensitive Activation Loop of the SARS-CoV-2 Spike Protein. ACS Omega 2021, 6, 7181-7185, doi:10.1021/acsomega.1c00363.

36. Lemmin, T.; Kalbermatter, D.; Harder, D.; Plattet, P.; Fotiadis, D. Structures and Dynamics of the Novel S1/S2 Protease Cleavage Site Loop of the SARS-CoV-2 Spike Glycoprotein. J. Struct. Biol. X 2020, 4, 100038, doi:10.1016/j.yjsbx.2020.100038.

37. Jaimes, J.A.; André, N.M.; Chappie, J.S.; Millet, J.K.; Whittaker, G.R. Phylogenetic Analysis and Structural Modeling of SARS-CoV-2 Spike Protein Reveals an Evolutionary Distinct and Proteolytically Sensitive Activation Loop. J. Mol. Biol. 2020, 432, 3309-3325, doi:10.1016/j.jmb.2020.04.009.

38. Gobeil, S.M.-C.; Janowska, K.; McDowell, S.; Mansouri, K.; Parks, R.; Manne, K.; Stalls, V.; Kopp, M.F.; Henderson, R.; Edwards, R.J.; et al. D614G Mutation Alters SARS-CoV-2 Spike Conformation and Enhances Protease Cleavage at the S1/S2 Junction. Cell Rep. 2021, 34, 108630, doi:10.1016/j.celrep.2020.108630.

39. Berman, H.M.; Westbrook, J.; Feng, Z.; Gilliland, G.; Bhat, T.N.; Weissig, H.; Shindyalov, I.N.; Bourne, P.E. The Protein Data Bank. Nucleic Acids Res. 2000, 28, 235-242, doi:10.1093/nar/28.1.235.

40. Timmer, J.C.; Zhu, W.; Pop, C.; Regan, T.; Snipas, S.J.; Eroshkin, A.M.; Riedl, S.J.; Salvesen, G.S. Structural and Kinetic Determinants of Protease Substrates. Nat. Struct. Mol. Biol. 2009, 16, 1101-1108, doi:10.1038/nsmb.1668.

41. Belizario, J.E.; Alves, J.; Garay-Malpartida, M.; Occhiucci, J.M. Coupling Caspase Cleavage and Proteasomal Degradation of Proteins Carrying PEST Motif. Curr. Protein Pept. Sci. 2008, 9, 210-220, doi:10.2174/138920308784534023.

42. Örd, M.; Faustova, I.; Loog, M. The Sequence at Spike S1/S2 Site Enables Cleavage by Furin and Phospho-Regulation in SARS-CoV2 but Not in SARS-CoV1 or MERS-CoV. Sci. Rep. 2020, 10, 16944, doi:10.1038/s41598-020-74101-0.

43. Gagliardi, P.A.; Primo, L. Irreversible Activation of Rho-Activated Kinases Resulted from Evolution of Proteolytic Sites within Disordered Regions in Coiled-Coil Domain. Mol. Biol. Evol. 2019, 36, 376-392, doi:10.1093/molbev/msy229.

44. Shaw, D.E.; Grossman, J.P.; Bank, J.A.; Batson, B.; Butts, J.A.; Chao, J.C.; Deneroff, M.M.; Dror, R.O.; Even, A.; Fenton, C.H.; et al. Anton 2: Raising the Bar for Performance and Programmability in a Special-Purpose Molecular Dynamics Supercomputer. In Proceedings of the SC14: International Conference for High Performance Computing, Networking, Storage and Analysis; IEEE: New Orleans, LA, USA, November 2014; pp. 41-53.

45. Huang, P.-S.; Ban, Y.-E.A.; Richter, F.; Andre, I.; Vernon, R.; Schief, W.R.; Baker, D. RosettaRemodel: A Generalized Framework for Flexible Backbone Protein Design. PLOS ONE 2011, 6, e24109, doi:10.1371/journal.pone.0024109. 
46. Mandell, D.J.; Coutsias, E.A.; Kortemme, T. Sub-Angstrom Accuracy in Protein Loop Reconstruction by Robotics-Inspired Conformational Sampling. Nat. Methods 2009, 6, 551-552, doi:10.1038/nmeth0809-551.

47. Basu, S.; Söderquist, F.; Wallner, B. Proteus: A Random Forest Classifier to Predict Disorder-to-Order Transitioning Binding Regions in Intrinsically Disordered Proteins. $J$. Comput. Aided Mol. Des. 2017, 1-14, doi:10.1007/s10822-017-0020-y.

48. Vankadari, N. Structure of Furin Protease Binding to SARS-CoV-2 Spike Glycoprotein and Implications for Potential Targets and Virulence. J. Phys. Chem. Lett. 2020, 11, 6655-6663, doi:10.1021/acs.jpclett.0c01698.

49. Basu, S.; Biswas, P. Salt-Bridge Dynamics in Intrinsically Disordered Proteins: A Trade-off between Electrostatic Interactions and Structural Flexibility. Biochim. Biophys. Acta BBA Proteins Proteomics 2018, 1866, 624-641, doi:10.1016/j.bbapap.2018.03.002.

50. Bandyopadhyay, A.; Basu, S. Criticality in the Conformational Phase Transition among Self-Similar Groups in Intrinsically Disordered Proteins: Probed by Salt-Bridge Dynamics. Biochim. Biophys. Acta BBA - Proteins Proteomics 2020, 1868, 140474, doi:10.1016/j.bbapap.2020.140474.

51. Ramachandran, G.N.; Ramakrishnan, C.; Sasisekharan, V. Stereochemistry of Polypeptide Chain Configurations. J. Mol. Biol. 1963, 7, 95-99.

52. Greaney, A.J.; Loes, A.N.; Crawford, K.H.D.; Starr, T.N.; Malone, K.D.; Chu, H.Y.; Bloom, J.D. Comprehensive Mapping of Mutations in the SARS-CoV-2 Receptor-Binding Domain That Affect Recognition by Polyclonal Human Plasma Antibodies. Cell Host Microbe 2021, 29, 463-476.e6, doi:10.1016/j.chom.2021.02.003.

53. Benton, D.J.; Wrobel, A.G.; Xu, P.; Roustan, C.; Martin, S.R.; Rosenthal, P.B.; Skehel, J.J.; Gamblin, S.J. Receptor Binding and Priming of the Spike Protein of SARS-CoV-2 for Membrane Fusion. Nature 2020, 588, 327-330, doi:10.1038/s41586-020-2772-0.

54. Seidah, N.G.; Mayer, G.; Zaid, A.; Rousselet, E.; Nassoury, N.; Poirier, S.; Essalmani, R.; Prat, A. The Activation and Physiological Functions of the Proprotein Convertases. Int. J. Biochem. Cell Biol. 2008, 40, 1111-1125, doi:10.1016/j.biocel.2008.01.030.

55. Dahms, S.O.; Arciniega, M.; Steinmetzer, T.; Huber, R.; Than, M.E. Structure of the Unliganded Form of the Proprotein Convertase Furin Suggests Activation by a SubstrateInduced Mechanism. Proc. Natl. Acad. Sci. 2016, 113, 11196-11201, doi:10.1073/pnas.1613630113.

56. Chen, X.; Zaro, J.L.; Shen, W.-C. Fusion Protein Linkers: Property, Design and Functionality. Adv. Drug Deliv. Rev. 2013, 65, 1357-1369, doi:10.1016/j.addr.2012.09.039.

57. Duckert, P.; Brunak, S.; Blom, N. Prediction of Proprotein Convertase Cleavage Sites. Protein Eng. Des. Sel. 2004, 17, 107-112, doi:10.1093/protein/gzh013.

58. Fedry, J.; Hurdiss, D.L.; Wang, C.; Li, W.; Obal, G.; Drulyte, I.; Du, W.; Howes, S.C.; van Kuppeveld, F.J.M.; Förster, F.; et al. Structural Insights into the Cross-Neutralization of SARS-CoV and SARS-CoV-2 by the Human Monoclonal Antibody 47D11. Sci. Adv. 2021, 7, eabf5632, doi:10.1126/sciadv.abf5632.

59. Casadevall, A.; Pirofski, L. The Convalescent Sera Option for Containing COVID-19. J. Clin. Invest. 2020, 130, 1545-1548, doi:10.1172/JCI138003. 
60. Song, W.; Gui, M.; Wang, X.; Xiang, Y. Cryo-EM Structure of the SARS Coronavirus Spike Glycoprotein in Complex with Its Host Cell Receptor ACE2. PLoS Pathog. 2018, 14, e1007236, doi:10.1371/journal.ppat.1007236.

61. Zhang, J.; Cai, Y.; Xiao, T.; Lu, J.; Peng, H.; Sterling, S.M.; Walsh, R.M.; Rits-Volloch, S.; Zhu, H.; Woosley, A.N.; et al. Structural Impact on SARS-CoV-2 Spike Protein by D614G Substitution. Science 2021, 372, 525-530, doi:10.1126/science.abf2303.

62. Eswar, N.; Webb, B.; Marti-Renom, M.A.; Madhusudhan, M. s.; Eramian, D.; Shen, M.; Pieper, U.; Sali, A. Comparative Protein Structure Modeling Using Modeller. Curr. Protoc. Bioinforma. 2006, 15, 5.6.1-5.6.30, doi:10.1002/0471250953.bi0506s15.

63. The UniProt Consortium UniProt: The Universal Protein Knowledgebase in 2021. Nucleic Acids Res. 2021, 49, D480-D489, doi:10.1093/nar/gkaa1100.

64. Alford, R.F.; Leaver-Fay, A.; Jeliazkov, J.R.; O’Meara, M.J.; DiMaio, F.P.; Park, H.; Shapovalov, M.V.; Renfrew, P.D.; Mulligan, V.K.; Kappel, K.; et al. The Rosetta All-Atom Energy Function for Macromolecular Modeling and Design. J. Chem. Theory Comput. 2017, 13, 3031-3048, doi:10.1021/acs.jctc.7b00125.

65. Kozakov, D.; Hall, D.R.; Xia, B.; Porter, K.A.; Padhorny, D.; Yueh, C.; Beglov, D.; Vajda, S. The ClusPro Web Server for Protein-Protein Docking. Nat. Protoc. 2017, 12, 255-278, doi:10.1038/nprot.2016.169.

66. Kozakov, D.; Brenke, R.; Comeau, S.R.; Vajda, S. PIPER: An FFT-Based Protein Docking Program with Pairwise Potentials. Proteins 2006, 65, 392-406, doi:10.1002/prot.21117.

67. Méndez, R.; Leplae, R.; De Maria, L.; Wodak, S.J. Assessment of Blind Predictions of Protein-Protein Interactions: Current Status of Docking Methods. Proteins Struct. Funct. Bioinforma. 2003, 52, 51-67, doi:10.1002/prot.10393.

68. Pierce, B.G.; Wiehe, K.; Hwang, H.; Kim, B.-H.; Vreven, T.; Weng, Z. ZDOCK Server: Interactive Docking Prediction of Protein-Protein Complexes and Symmetric Multimers. Bioinforma. Oxf. Engl. 2014, 30, 1771-1773, doi:10.1093/bioinformatics/btu097.

69. Hubbard S, Thornton J (1993) NACCESS. Computer Program, Department of Biochemistry and Molecular Biology, University College London. - Open Access Library Available online: http://www.oalib.com/references/5299711 (accessed on 1 March 2017).

70. Lee, B.; Richards, F.M. The Interpretation of Protein Structures: Estimation of Static Accessibility. J. Mol. Biol. 1971, 55, 379-400.

71. Basu, S.; Wallner, B. Finding Correct Protein-Protein Docking Models Using ProQDock. Bioinforma. Oxf. Engl. 2016, 32, i262-i270, doi:10.1093/bioinformatics/btw257.

72. Banerjee, R.; Sen, M.; Bhattacharya, D.; Saha, P. The Jigsaw Puzzle Model: Search for Conformational Specificity in Protein Interiors. J. Mol. Biol. 2003, 333, 211-226.

73. Basu, S.; Bhattacharyya, D.; Banerjee, R. Mapping the Distribution of Packing Topologies within Protein Interiors Shows Predominant Preference for Specific Packing Motifs. BMC Bioinformatics 2011, 12, 195, doi:10.1186/1471-2105-12-195.

74. Basu, S.; Bhattacharyya, D.; Banerjee, R. Self-Complementarity within Proteins: Bridging the Gap between Binding and Folding. Biophys. J. 2012, 102, 2605-2614, doi:10.1016/j.bpj.2012.04.029.

75. Basu, S.; Bhattacharyya, D.; Banerjee, R. Applications of Complementarity Plot in Error Detection and Structure Validation of Proteins. Indian J. Biochem. Biophys. 2014, 51, 188200. 
76. Lawrence, M.C.; Colman, P.M. Shape Complementarity at Protein/Protein Interfaces. J. Mol. Biol. 1993, 234, 946-950, doi:10.1006/jmbi.1993.1648.

77. Basu, S. CPdock: The Complementarity Plot for Docking of Proteins: Implementing MultiDielectric Continuum Electrostatics. J. Mol. Model. 2017, 24, 8, doi:10.1007/s00894-0173546-y.

78. SC (CCP4: Supported Program) - CCP4Docs Documentation Available online: https://www.ccp4.ac.uk/html/sc.html (accessed on 11 November 2021).

79. Berendsen, H.J.C.; van der Spoel, D.; van Drunen, R. GROMACS: A Message-Passing Parallel Molecular Dynamics Implementation. Comput. Phys. Commun. 1995, 91, 43-56, doi:10.1016/0010-4655(95)00042-E.

80. Siu, S.W.I.; Pluhackova, K.; Böckmann, R.A. Optimization of the OPLS-AA Force Field for Long Hydrocarbons. J. Chem. Theory Comput. 2012, 8, 1459-1470, doi:10.1021/ct200908r.

81. Grant, O.C.; Montgomery, D.; Ito, K.; Woods, R.J. Analysis of the SARS-CoV-2 Spike Protein Glycan Shield: Implications for Immune Recognition. bioRxiv 2020, 2020.04.07.030445, doi:10.1101/2020.04.07.030445.

82. Hess, B.; Bekker, H.; Berendsen, H.J.C.; Fraaije, J.G.E.M. LINCS: A Linear Constraint Solver for Molecular Simulations. J. Comput. Chem. 1997, 18, 1463-1472, doi:10.1002/(SICI)1096-987X(199709)18:12<1463::AID-JCC4>3.0.CO;2-H.

83. Essmann, U.; Perera, L.; Berkowitz, M.L.; Darden, T.; Lee, H.; Pedersen, L.G. A Smooth Particle Mesh Ewald Method. J. Chem. Phys. 1995, 103, 8577-8593, doi:10.1063/1.470117.

84. Berendsen, H.J.C.; Postma, J.P.M.; van Gunsteren, W.F.; DiNola, A.; Haak, J.R. Molecular Dynamics with Coupling to an External Bath. J. Chem. Phys. 1984, 81, 3684-3690, doi:10.1063/1.448118.

85. Parrinello, M.; Rahman, A. Polymorphic Transitions in Single Crystals: A New Molecular Dynamics Method. J. Appl. Phys. 1981, 52, 7182-7190, doi:10.1063/1.328693.

86. Basu, S.; Mukharjee, D. Salt-Bridge Networks within Globular and Disordered Proteins: Characterizing Trends for Designable Interactions. J. Mol. Model. 2017, 23, 206, doi:10.1007/s00894-017-3376-y.

87. Musafia, B.; Buchner, V.; Arad, D. Complex Salt Bridges in Proteins: Statistical Analysis of Structure and Function. J. Mol. Biol. 1995, 254, 761-770, doi:10.1006/jmbi.1995.0653.

88. Edelman, G.M.; Gally, J.A. Degeneracy and Complexity in Biological Systems. Proc. Natl. Acad. Sci. 2001, 98, 13763-13768, doi:10.1073/pnas.231499798.

89. Guerois, R.; Nielsen, J.E.; Serrano, L. Predicting Changes in the Stability of Proteins and Protein Complexes: A Study of More than 1000 Mutations. J. Mol. Biol. 2002, 320, 369387, doi:10.1016/S0022-2836(02)00442-4.

90. Schymkowitz, J.; Borg, J.; Stricher, F.; Nys, R.; Rousseau, F.; Serrano, L. The FoldX Web Server: An Online Force Field. Nucleic Acids Res. 2005, 33, W382-388, doi:10.1093/nar/gki387.

91. Li, M.; Simonetti, F.L.; Goncearenco, A.; Panchenko, A.R. MutaBind Estimates and Interprets the Effects of Sequence Variants on Protein-Protein Interactions. Nucleic Acids Res. 2016, 44, W494-W501, doi:10.1093/nar/gkw374. 
92. Buß, O.; Rudat, J.; Ochsenreither, K. FoldX as Protein Engineering Tool: Better Than Random Based Approaches? Comput. Struct. Biotechnol. J. 2018, 16, 25-33, doi:10.1016/j.csbj.2018.01.002.

93. Broom, A.; Jacobi, Z.; Trainor, K.; Meiering, E.M. Computational Tools Help Improve Protein Stability but with a Solubility Tradeoff. J. Biol. Chem. 2017, 292, 14349-14361, doi:10.1074/jbc.M117.784165.

94. Vanhee, P.; Verschueren, E.; Baeten, L.; Stricher, F.; Serrano, L.; Rousseau, F.; Schymkowitz, J. BriX: A Database of Protein Building Blocks for Structural Analysis, Modeling and Design. Nucleic Acids Res 2011, 39, doi:10.1093/nar/gkq972.

95. Kamisetty, H.; Ramanathan, A.; Bailey-Kellogg, C.; Langmead, C.J. Accounting for Conformational Entropy in Predicting Binding Free Energies of Protein-Protein Interactions. Proteins 2011, 79, 444-462, doi:10.1002/prot.22894.

96. Brackley, C.A.; Liebchen, B.; Michieletto, D.; Mouvet, F.; Cook, P.R.; Marenduzzo, D. Ephemeral Protein Binding to DNA Shapes Stable Nuclear Bodies and Chromatin Domains. Biophys. J. 2017, 112, 1085-1093, doi:10.1016/j.bpj.2017.01.025.

97. Zanotti, J.-M.; Gibrat, G.; Bellissent-Funel, M.-C. Hydration Water Rotational Motion as a Source of Configurational Entropy Driving Protein Dynamics. Crossovers at 150 and 220 K. Phys. Chem. Chem. Phys. 2008, 10, 4865-4870, doi:10.1039/B808217K.

98. Angell, C.A. Entropy and Fragility in Supercooling Liquids. J. Res. Natl. Inst. Stand. Technol. 1997, 102, 171-185, doi:10.6028/jres.102.013.

99. Zhou, T.; Tsybovsky, Y.; Gorman, J.; Rapp, M.; Cerutti, G.; Chuang, G.-Y.; Katsamba, P.S.; Sampson, J.M.; Schön, A.; Bimela, J.; et al. Cryo-EM Structures of SARS-CoV-2 Spike without and with ACE2 Reveal a PH-Dependent Switch to Mediate Endosomal Positioning of Receptor-Binding Domains. Cell Host Microbe 2020, 28, 867-879.e5, doi:10.1016/j.chom.2020.11.004.

100. Cueno, M.E.; Ueno, M.; Iguchi, R.; Harada, T.; Miki, Y.; Yasumaru, K.; Kiso, N.; Wada, K.; Baba, K.; Imai, K. Insights on the Structural Variations of the Furin-Like Cleavage Site Found Among the December 2019-July 2020 SARS-CoV-2 Spike Glycoprotein: A Computational Study Linking Viral Evolution and Infection. Front. Med. 2021, 8, 240, doi:10.3389/fmed.2021.613412.

101. Jones, D.T.; Cozzetto, D. DISOPRED3: Precise Disordered Region Predictions with Annotated Protein-Binding Activity. Bioinformatics 2015, 31, 857-863, doi:10.1093/bioinformatics/btu744.

102. Ishida, T.; Kinoshita, K. PrDOS: Prediction of Disordered Protein Regions from Amino Acid Sequence. Nucleic Acids Res. 2007, 35, W460-464, doi:10.1093/nar/gkm363.

103. Mészáros, B.; Erdős, G.; Dosztányi, Z. IUPred2A: Context-Dependent Prediction of Protein Disorder as a Function of Redox State and Protein Binding. Nucleic Acids Res. 2018, 46, W329-W337, doi:10.1093/nar/gky384.

104. Katuwawala, A.; Kurgan, L. Comparative Assessment of Intrinsic Disorder Predictions with a Focus on Protein and Nucleic Acid-Binding Proteins. Biomolecules 2020, 10, 1636, doi:10.3390/biom10121636.

105. Katuwawala, A.; Oldfield, C.J.; Kurgan, L. Accuracy of Protein-Level Disorder Predictions. Brief. Bioinform. 2020, 21, 1509-1522, doi:10.1093/bib/bbz100. 
106. Edgar, R.C. MUSCLE: A Multiple Sequence Alignment Method with Reduced Time and Space Complexity. BMC Bioinformatics 2004, 5, 113, doi:10.1186/1471-2105-5-113.

107. Wong, E.T.C. Electrostatics in Intrinsically Disordered Proteins, University of British Columbia, 2012.

108. Liu, C.; Wang, T.; Bai, Y.; Wang, J. Electrostatic Forces Govern the Binding Mechanism of Intrinsically Disordered Histone Chaperones. PLOS ONE 2017, 12, e0178405, doi:10.1371/ journal.pone.0178405.

109. Basu, S.; Bahadur, R.P. Conservation and Coevolution Determine Evolvability of Different Classes of Disordered Residues in Human Intrinsically Disordered Proteins. Proteins Struct. Funct. Bioinforma. n/a, doi:10.1002/prot.26261.

110. Wong, E.T.C.; Na, D.; Gsponer, J. On the Importance of Polar Interactions for Complexes Containing Intrinsically Disordered Proteins. PLoS Comput. Biol. 2013, 9, e1003192, doi:10.1371/journal.pcbi.1003192.

111. Tedeschi, G.; Salladini, E.; Santambrogio, C.; Grandori, R.; Longhi, S.; Brocca, S. Conformational Response to Charge Clustering in Synthetic Intrinsically Disordered Proteins. Biochim. Biophys. Acta BBA - Gen. Subj. 2018, 1862, 2204-2214, doi:10.1016/j.bbagen.2018.07.011.

112. Cui, H.; Eltoukhy, L.; Zhang, L.; Markel, U.; Jaeger, K.-E.; Davari, M.D.; Schwaneberg, U. Less Unfavorable Salt Bridges on the Enzyme Surface Result in More Organic Cosolvent Resistance. Angew. Chem. Int. Ed. 2021, 60, 11448-11456, doi:10.1002/anie.202101642.

113. Richards, F.M.; Kundrot, C.E. Identification of Structural Motifs from Protein Coordinate Data: Secondary Structure and First-Level Supersecondary Structure. Proteins 1988, 3, 7184, doi:10.1002/prot.340030202.

114. Behe, M.J.; Lattman, E.E.; Rose, G.D. The Protein-Folding Problem: The Native Fold Determines Packing, but Does Packing Determine the Native Fold? Proc. Natl. Acad. Sci. U. S. A. 1991, 88, 4195-4199.

115. Rose, G.D.; Roy, S. Hydrophobic Basis of Packing in Globular Proteins. Proc. Natl. Acad. Sci. U. S. A. 1980, 77, 4643-4647.

116. Takano, K.; Yamagata, Y.; Yutani, K. A General Rule for the Relationship between Hydrophobic Effect and Conformational Stability of a Protein: Stability and Structure of a Series of Hydrophobic Mutants of Human Lysozyme. J. Mol. Biol. 1998, 280, 749-761, doi:10.1006/jmbi.1998.1906.

117. Demchenko, A.P. Structural Relaxation in Protein Molecules Studied by Fluorescence Spectroscopy. J. Mol. Struct. 1984, 114, 45-48, doi:10.1016/S0022-2860(84)87201-4.

118. Ramachandran Plot - an Overview | ScienceDirect Topics Available online: https://www.sciencedirect.com/topics/biochemistry-genetics-and-molecular-biology/ ramachandran-plot (accessed on 5 November 2021).

119. Heinig, M.; Frishman, D. STRIDE: A Web Server for Secondary Structure Assignment from Known Atomic Coordinates of Proteins. Nucleic Acids Res. 2004, 32, W500-W502, doi:10.1093/nar/gkh429.

120. Kleywegt, G.J.; Jones, T.A. Phi/Psi-Chology: Ramachandran Revisited. Structure 1996, 4, 1395-1400, doi:10.1016/S0969-2126(96)00147-5.

121. Ramachandran, G.N.; Sasisekharan, V. Conformation of Polypeptides and Proteins. Adv. Protein Chem. 1968, 23, 283-438. 
122. Zhou, A.Q.; O’Hern, C.S.; Regan, L. Revisiting the Ramachandran Plot from a New Angle. Protein Sci. Publ. Protein Soc. 2011, 20, 1166-1171, doi:10.1002/pro.644.

123. Laskowski, R.A.; MacArthur, M.W.; Moss, D.S.; Thornton, J.M. PROCHECK: A Program to Check the Stereochemical Quality of Protein Structures. J. Appl. Crystallogr. 1993, 26, 283-291, doi:10.1107/S0021889892009944.

124. Michel Espinoza-Fonseca, L.; Kast, D.; Thomas, D.D. Molecular Dynamics Simulations Reveal a Disorder-to-Order Transition on Phosphorylation of Smooth Muscle Myosin. Biophys. J. 2007, 93, 2083-2090, doi:10.1529/biophysj.106.095802. 
A.

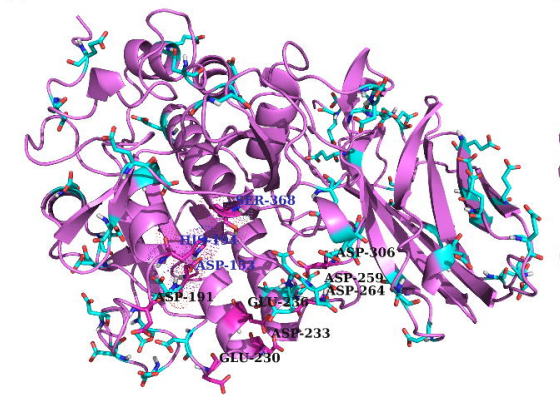

B.

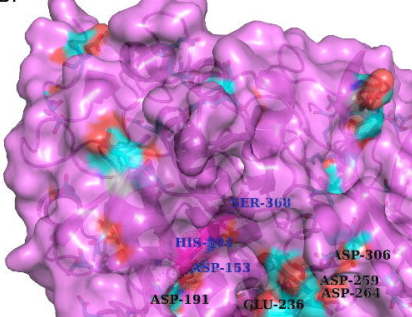
ASP-191 GuU-236 ASP-264 
A.

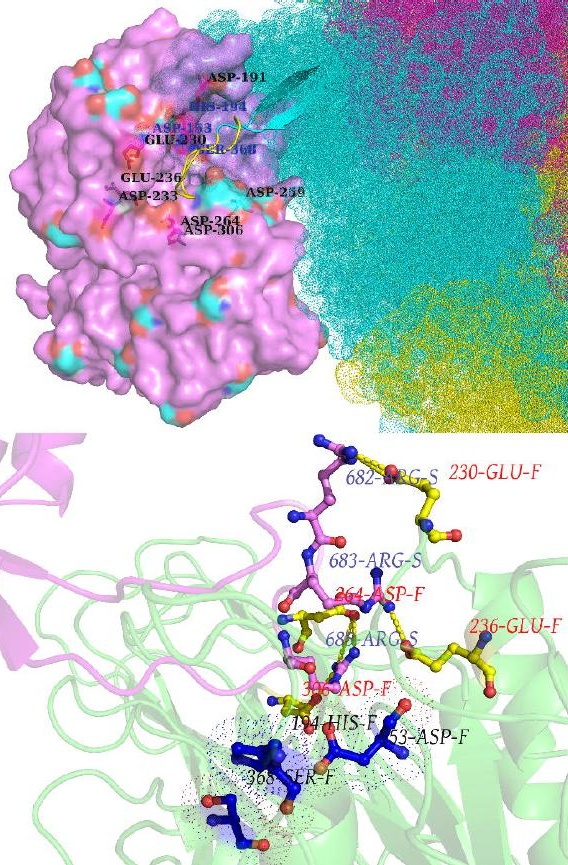


A. Anionic Triad \& Catalytic Triad in correlated movements in RR1 (300 ns)

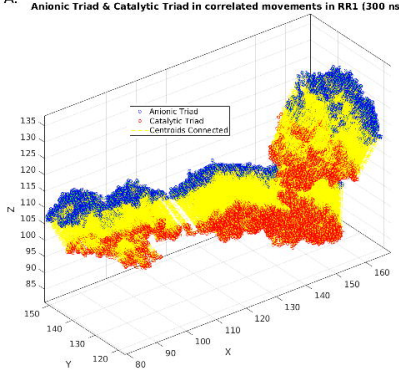

B.

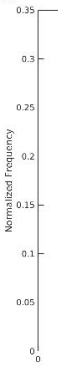

The constrained Inter-triad Angle ( $\varepsilon$ ) in Spike-bound Furin

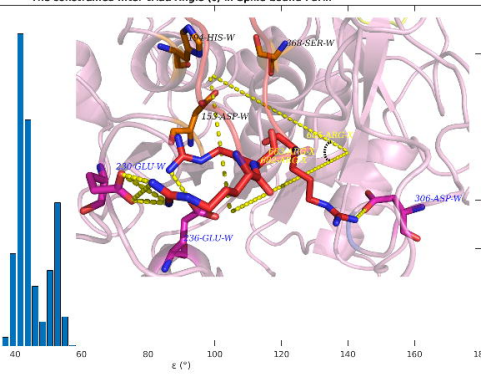




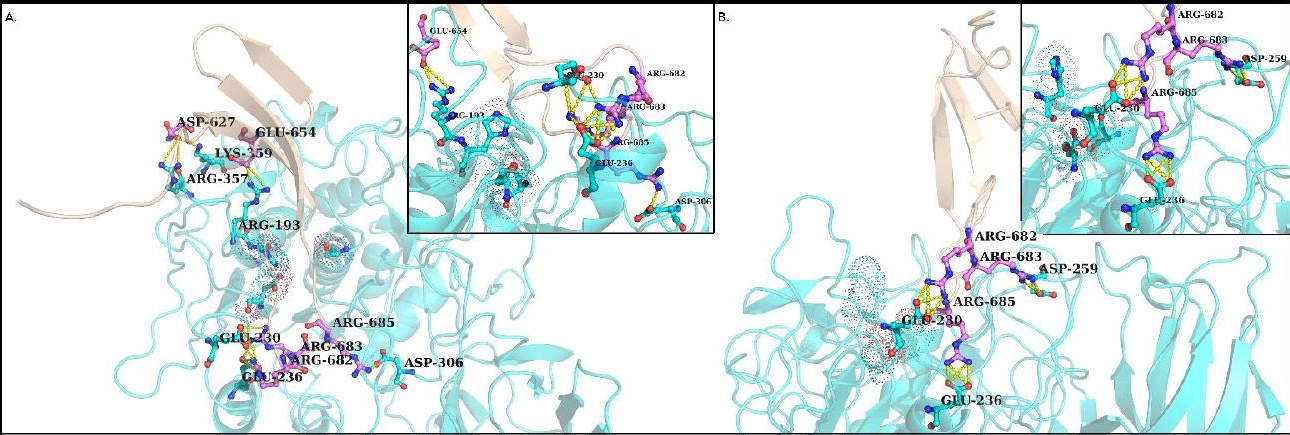




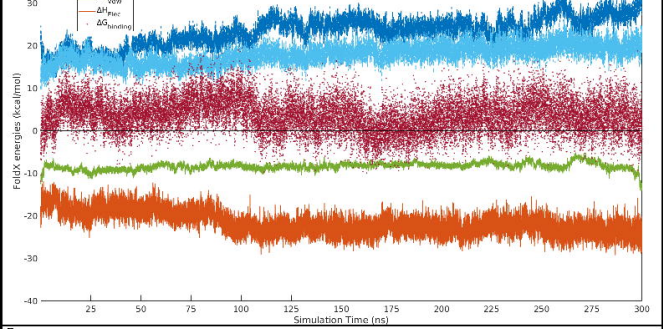

B.

Furin-Spike (FoldX) Interaction Energies: $\mathrm{ZR}_{\mathrm{CoV} 2}$ (MD $100 \mathrm{~ns}$ )

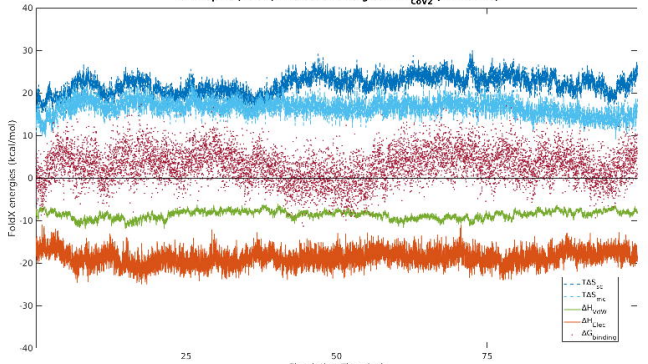

Simulation Time (ns)

C.

Furin-Spike (FoldX) Interaction Energies: ZR1 cov (MD $100 \mathrm{~ns}$ )

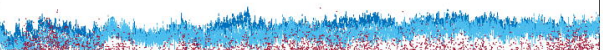

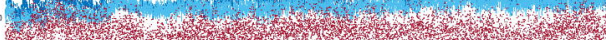
3. (1) nomplimpov

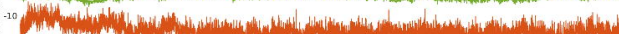
$-20$ 

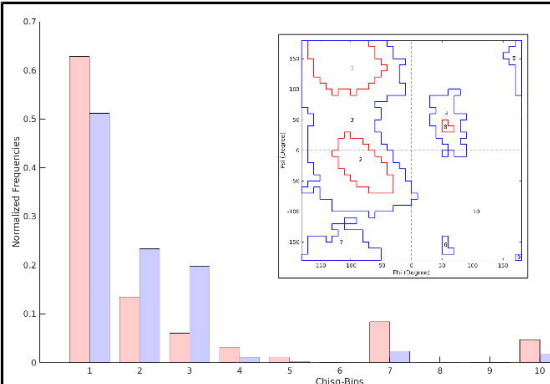

${ }^{5}$ Chisq-Eins 